\title{
Constraining the Metallicities, Ages, Star Formation Histories, and Ionizing Continua of Extragalactic Massive Star Populations*
}

\author{
J. Chisholm ${ }^{1}$ (i), J. R. Rigby ${ }^{2}$ (D) M. Bayliss ${ }^{3}$ (D), D. A. Berg ${ }^{4}$ (D) H. Dahle ${ }^{5}$ (D) M. Gladders ${ }^{6}$, and K. Sharon ${ }^{7}$ (D) \\ ${ }^{1}$ University of California—Santa Cruz, 1156 High St, Santa Cruz, CA 95064, USA; jochisho@ucsc.edu \\ ${ }^{2}$ Observational Cosmology Lab, NASA Goddard Space Flight Center, 8800 Greenbelt Rd, Greenbelt, MD 20771, USA \\ ${ }^{3}$ MIT Kavli Institute for Astrophysics and Space Research, 77 Massachusetts Ave, Cambridge, MA 02139, USA \\ ${ }^{4}$ Department of Astronomy, The Ohio State University, 140 W. 18th Ave, Columbus, OH 43202, USA \\ ${ }^{5}$ Institute of Theoretical Astrophysics, University of Oslo, P.O. Box 1029, Blindern, NO-0315 Oslo, Norway \\ ${ }^{6}$ Kavli Institute for Cosmological Physics, University of Chicago, 5640 South Ellis Ave., Chicago, IL 60637, USA \\ ${ }^{7}$ Department of Astronomy, University of Michigan, 500 Church St, Ann Arbor, MI 48109, USA \\ Received 2019 April 19; revised 2019 June 13; accepted 2019 June 21; published 2019 September 16
}

\begin{abstract}
We infer the properties of massive star populations using the far-ultraviolet stellar continua of 61 star-forming galaxies: 42 at low redshift observed with the Hubble Space Telescope and 19 at $z \sim 2$ from the MEGaSaURA sample. We fit each stellar continuum with a linear combination of up to 50 single-age and single-metallicity STARBURST99 models. From these fits, we derive light-weighted ages and metallicities, which agree with stellar wind and photospheric spectral features, and infer the spectral shapes and strengths of the ionizing continua. Inferred light-weighted stellar metallicities span $0.05-1.5 Z_{\odot}$ and are similar to the measured nebular metallicities. We quantify the ionizing continua using the ratio of the ionizing flux at $900 \AA$ to the non-ionizing flux at $1500 \AA$ and demonstrate the evolution of this ratio with stellar age and metallicity using theoretical single-burst models. These single-burst models only match the inferred ionizing continua of half of the sample, while the other half are described by a mixture of stellar ages. Mixed-age populations produce stronger and harder ionizing spectra than continuous star formation histories, but, contrary to previous studies that assume constant star formation, have similar stellar and nebular metallicities. Stellar population age and metallicity affect the far-UV continua in different and distinguishable ways; assuming a constant star formation history diminishes the diagnostic power. Finally, we provide simple prescriptions to determine the ionizing photon production efficiency $\left(\xi_{\text {ion }}\right)$ from the stellar population properties. The $\xi_{\text {ion }}$ inferred from the observed star-forming galaxies has a range of $\log \left(\xi_{\text {ion }}\right)=24.4-25.7 \mathrm{~Hz} \mathrm{erg}^{-1}$ that depends on the stellar population age, metallicity, star formation history, and contributions from binary star evolution. These stellar population properties must be observationally determined to accurately determine the number of ionizing photons generated by massive stars.
\end{abstract}

Key words: binaries: general - dark ages, reionization, first stars - galaxies: abundances - galaxies: starburst

Supporting material: tar.gz file

\section{Introduction}

O-type stars, which have masses $>15 M_{\odot}$ and lifetimes $<10 \mathrm{Myr}$, are the only main-sequence stars hot enough to generate a significant number of ionizing photons $(\lambda<912 \AA)$. These photons ionize hydrogen in the interstellar medium, powering the nebular emission lines that reveal the physical state (Strömgren 1939; Seyfert 1943; Baldwin et al. 1991) and chemical evolution (Tinsley 1980) of star-forming galaxies. The emission lines trace the most recent star formation and measure the rate at which stars form (Kennicutt 1998; Kennicutt \& Evans 2012). These observations describe how galaxies build up their stellar mass (Brinchmann et al. 2004; Elbaz et al. 2007; Noeske et al. 2007) and how star formation evolves with cosmic time (Madau et al. 1999; Madau \& Dickinson 2014). Massive stars affect more than just their host galaxies: the ionizing photons produced by the earliest stars may have been sufficient to reionize the universe (Ouchi et al. 2009; Robertson et al. 2013, 2015; Finkelstein et al. 2019). Ionizing photons from massive stars generate the fundamental observables that describe the formation and evolution of star-

\footnotetext{
* Based on observations made with the NASA/ESA Hubble Space Telescope, obtained from the Data Archive at the Space Telescope Science Institute, which is operated by the Association of Universities for Research in Astronomy, Inc., under NASA contract NAS 5-26555.
}

forming galaxies. As such, determining how stars produce ionizing photons is fundamental to understanding galaxy formation and evolution.

Stellar ionizing photons are challenging to directly observe because neutral hydrogen within galaxies efficiently absorbs ionizing photons. Nearly all inferences about the flux and spectral shape of the stellar ionizing continua have been made either from emission lines that have been reprocessed through nebular gas adjacent to massive stars, or from the technique of stellar population synthesis. Stellar population synthesis constructs a model stellar spectrum by first determining a hypothetical stellar population (with a given age, composition, and star formation history) and then creating a theoretical spectrum of that stellar population using model stellar atmospheres. The stellar age, metallicity, and star formation history are inferred by constructing models with a range of these parameters and using statistical methods to determine which population values best match the observed spectrum. Large libraries of rest-frame optical spectra, from surveys such as the Sloan Digital Sky Survey (Alam et al. 2015), have revolutionized population synthesis at optical wavelengths (Bruzual \& Charlot 2003; Maraston 2005; Conroy 2013).

Stellar population synthesis of the most massive stars can, in principle, constrain the ionizing continua of massive stars. However, massive stars have largely featureless optical spectra 
that do not change appreciably with stellar metallicity or age. Therefore, optical stellar population synthesis has a temporal resolution on the order of $10-100 \mathrm{Myr}$ when B-stars, with significant Balmer absorption features, begin to appear in optical spectra. In contrast, the O-stars that produce the majority of the ionizing photons have much shorter lifetimes of 2-10 Myr.

The ideal wavelength range to capture the rapid temporal evolution of massive stars is the rest-frame far-ultraviolet (FUV). The FUV contains spectral features of massive stars, namely stellar wind lines (Walborn et al. 1985, 2002; Howarth \& Prinja 1989; Lamers \& Cassinelli 1999; Pellerin et al. 2002), which have been observed in star-forming galaxies over most of cosmic time (Kinney et al. 1993; Heckman et al. 1998; Pettini et al. 2002; Leitherer et al. 2011; Steidel et al. 2016; Rigby et al. 2018b). The shape and strength of these spectral features strongly depend on both the ages and metallicities of the stellar populations (Leitherer et al. 1995, 1999; Smith et al. 2002), enabling FUV spectral synthesis to determine the population properties of massive stars.

Both stellar physics and stellar population properties dictate the production of ionizing photons. In spectral population synthesis, the stellar models amass the complicated underlying stellar physics of the individual stellar properties and evolution that lead to the observed stellar continuum. These vital stellar physical properties include the initial mass function (IMF; Salpeter 1955; Kroupa 2001; Chabrier 2003), stellar rotation (Meynet \& Maeder 2000; Levesque et al. 2012; Leitherer et al. 2014), and the stellar evolution tracks that may include interactions among binary stars (Meynet et al. 1994; Leitherer et al. 1995; Eldridge \& Stanway 2009; Stanway et al. 2016). Ultimately, stellar spectral population synthesis is founded upon the individual stellar models. The success or failure of the stellar synthesis relies upon the models properly incorporating the crucial stellar physics.

Predominantly, this paper focuses on using stellar models to constrain stellar population properties such as age, metallicity, and star formation history. We then use these properties to infer their ionizing continua. More massive stars must be hotter to counteract their intense gravity and remain in hydrostatic equilibrium. Increased stellar temperatures produce bluer spectra and fully ionized stellar atmospheres. Both effects lead to the production of a copious amount of ionizing photons. These massive stars rapidly exhaust the hydrogen in their cores and have much shorter lifetimes than cooler stars. Consequently, ionizing photons are only produced by the youngest and most massive stars. Further, because hydrogen is highly ionized in their photospheres, metals are the main opacity source of ionizing photons in massive stars. Thus, lowermetallicity stars produce significantly more ionizing photons than stars of similar ages but higher metallicities. The stellar age and metallicity must be observationally constrained to determine the number of ionizing photons generated by massive stars.

The 2-10 Myr lifetimes of the most massive stars are only $1 \%-10 \%$ of the dynamical timescales of galaxies. Thus, the relative proportion of massive stars depends on when stars were formed and how many stars formed at each epoch. This is referred to as the star formation history. A starburst galaxy is typically defined as recently forming a large fraction of the total stellar mass, so its star formation history is typically assumed to be nearly a delta function of a single burst (McQuinn et al. 2010a). Meanwhile, the entire disks of normal star-forming galaxies have more moderate, nearly constant star formation histories that generate new massive stars at a nearly constant rate (Leitherer et al. 1995). A constant star formation history always has a component of young massive stars capable of producing ionizing photons that is diluted by the older population. Nature is unlikely to comply with these simplified star formation histories, and the true star formation histories are assuredly somewhere between these two extremes (McQuinn et al. 2010b). To understand the relative strength of the youngest stellar populations and their role in producing ionizing photons, there must be an observationally motivated method to determine the star formation history.

In this paper, we perform FUV stellar population synthesis to constrain the age, metallicity, star formation history, and ionizing continua of extragalactic massive star populations. We fit the non-ionizing FUV continua of a sample of 61 low and moderate redshift star-forming galaxies as a linear combination of single-age, fully theoretical stellar continuum models. We infer the light-weighted ages, metallicities, and ionizing continua of the massive star populations from these fits. We compare the stellar and nebular metallicities (Section 5.1) and explore the inferred ionizing continua of the stellar populations (Section 5.2). The star formation histories are derived by comparing the inferred stellar continuum fits to single-burst models (Section 5.3). We test the observational differences between populations that contain binary stars (Section 5.4) and illustrate how the stellar continuum fits predict the total number of ionizing photons (Section 5.7).

Throughout this paper we follow the literature convention and assume that stellar solar metallicity is 0.02 (Leitherer et al. 1999, 2010; Stanway \& Eldridge 2018b). It is debated whether solar abundance is actually higher or lower than this value (Nieva \& Przybilla 2012; Villante et al. 2014), but we retain the 0.02 value used in stellar models because it determines the stellar evolution tracks and stellar wind profiles. We take the solar gas-phase metallicity to be $12+\log (\mathrm{O} / \mathrm{H})=8.69$ and $Z_{\odot}^{\text {neb }}=0.0142$ (Asplund et al. 2009). All spectra and flux densities are plotted and quoted in $F_{\lambda}$ units ( $\operatorname{erg~s}^{-1} \mathrm{~cm}^{-2} \AA^{-1}$ ). The equivalent widths of absorption lines are defined to be positive; emission lines are defined to be negative.

\section{Data \\ 2.1. Moderate-redshift Galaxies}

\subsubsection{MEGaSaURA Data}

Here we predominately display spectra of 19 star-forming galaxies from project MEGaSaURA: the Magellan Evolution of Galaxies Spectroscopic and Ultraviolet Reference Atlas (Rigby et al. 2018a). The extended MEGaSaURA sample includes the brightest southern lensed galaxies found in the Red-sequence Cluster Survey (RCS; Gladders \& Yee 2005), the Sloan Giant Arcs Survey (SGAS; Bayliss et al. 2011), the South Pole Telescope (SPT; Schaffer et al. 2011), and the ESA Planck survey (Planck Collaboration et al. 2014, 2016). These surveys found $z \sim 2$ star-forming galaxies behind massive foreground galaxy clusters. The mass of the foreground clusters magnifies, stretches, and amplifies the light from the background starforming galaxies, enabling high signal-to-noise ratio $(\mathrm{S} / \mathrm{N})$ and moderate spectral resolution rest-frame FUV observations of $z \sim 2$ galaxies with ground-based telescopes. MEGaSaURA is the ideal individual galaxy, FUV stellar spectral reference sample. 
MEGaSaURA spectra were taken with the Magellan Echellette (MagE) Spectrograph (Marshall et al. 2008) on the Magellan telescopes. Thirteen of the 19 spectra presented here were included in the original MEGaSaURA data release (Rigby et al. 2018a). Additionally, we include six galaxies from the upcoming expanded MEGaSaURA sample (J. R. Rigby et al. 2019, in preparation): the Sunburst Arc (Dahle et al. 2016; Rivera-Thorsen et al. 2017), SPT 0142, SPT 0310, SPT 0356, PSZ 0441, and SPT 2325. We only include MEGaSaURA spectra with $\mathrm{S} / \mathrm{N}>5$ per resolution element and without active galactic nucleus (AGN) signatures, which means that we exclude $\mathrm{S} 1050+0017$ (low S/N) and S2243-0935 (rest-frame optical AGN emission lines) from the original sample. The data reduction and full spectra of the original MEGaSaURA spectra were presented in Rigby et al. (2018a).

The MEGaSaURA galaxies span a redshift range $1.6<z<$ 3.1 (see Table 3), with a rest-frame FUV spectral coverage of $1220-1950 \AA$ for all 19 galaxies at a median spectral resolution of $R=3300\left(90 \mathrm{~km} \mathrm{~s}^{-1}\right.$, or $0.5 \AA$ at $\left.1500 \AA\right)$ and $\mathrm{S} / \mathrm{N}=21$ (Rigby et al. 2018a). The spectra were corrected for Milky Way reddening in the observed frame using the Cardelli et al. (1989) attenuation curve and the dust maps from Green et al. (2015). We normalized the spectra to the median of the flux in the line-free region of $1267-1276 \AA$ in the rest frame. The MEGaSaURA spectra contain all of the strong stellar features that constrain the stellar fits at a high $\mathrm{S} / \mathrm{N}$ with a resolution similar to the stellar continuum models. Due to the superior combination of wavelength coverage and sensitivity, we use the MEGaSaURA sample as our main sample instead of the Hubble Space Telescope (HST)/Cosmic Origins Spectrograph (COS) sample introduced below.

\subsubsection{Moderate-redshift Stacked Data}

While the individual MEGaSaURA spectra have high $\mathrm{S} / \mathrm{N}$, many of the important stellar features are extremely weak. By averaging many observations together (often called "stacking"), the $\mathrm{S} / \mathrm{N}$ increases by a factor of $\sqrt{N}$, where $N$ is the number of spectra included in the stack. Consequently, a composite provides an average spectrum of an ensemble of galaxies at an extremely high $\mathrm{S} / \mathrm{N}$ (see Section 5.5).

This stacking procedure has demonstrated the average FUV spectrum of galaxies at moderate redshifts (Shapley et al. 2003; Steidel et al. 2016, 2018; Rigby et al. 2018b). We use two recent stacks: (1) the MEGaSaURA lensed galaxies (Rigby et al. 2018 b) and (2) a stack of 30 star-forming field galaxies at $z \sim 2.4$ from the Keck Baryonic Structure Survey (KBSS; Steidel et al. 2016). The MEGaSaURA stack has a peak $\mathrm{S} / \mathrm{N}$ of 104 per spectral resolution with an average spectral resolution of $R=3300$ in the rest-frame wavelength range of 900-3000 A. The Steidel et al. (2016) stack has a peak S/N of 38 per spectral resolution at a resolution of $R=1400$ and rest-frame wavelength coverage between 1000 and $2200 \AA$.

\subsection{Low-redshift Galaxies}

Our low-redshift sample consists of spectra from recent observations of two low-metallicity galaxies (PID: HST-GO15099, PI: Chisholm) and the compilation from Chisholm et al. (2016), which are 40 local star-forming galaxies at $0.0007<z<0.1816$ with high $\mathrm{S} / \mathrm{N}$ observations using the COS (Green et al. 2012) on the HST. The data were compiled from eight different $H S T$ programs, and we include the $H S T$ program IDs and references in Table 4. The spectra were processed through CalCOS v2.20.1, reduced following the procedures in Wakker et al. (2015), binned by 20 pixels $(0.2 \AA$, or $48 \mathrm{~km} \mathrm{~s}^{-1}$ at $1240 \AA$ ), and convolved to the resolution of the STARBURST99 models ( $0.4 \AA$ ). We normalized the spectra near $1270 \AA$, similar to the MEGaSaURA observations. These spectra are also corrected for Milky Way reddening in the observed frame. The COS observations are typically only made with one grating $(\mathrm{G} 130 \mathrm{M})$, such that the average rest-frame wavelength coverage is from 1150 to $1450 \AA$. This spectral regime contains many, but not all, of the stellar features that define the stellar age and metallicity (see Section 4). As such, we display the MEGaSaURA sample throughout this paper rather than the narrow $H S T / C O S$ wavelength range.

\subsection{Host Galaxy Properties}

The 61 galaxies studied here sample a wide range in host galaxy properties. Tables 3 and 4 give literature nebular metallicity values, measured as $12+\log (\mathrm{O} / \mathrm{H})$ and referred to as $Z_{\text {neb}}$, which were determined using rest-frame optical emission lines. The "gold standard" nebular metallicity method, the direct method, uses the temperature-sensitive [O III] $4363 \AA$ emission line to determine the emission-line emissivities, which directly translate into oxygen abundances. However, [O III] $4363 \AA$ is a weak emission line that is challenging to observe in faint galaxies and is substantially weaker in higher metallicity regions. In the absence of [O III] $4363 \AA$ detections, calibration techniques have been developed using strong nebular emission lines to infer the ionization structure and nebular metallicity. These strong-line abundances are easily observed, but the inferred absolute abundances from different calibration methods can be discrepant by as much as 0.7 dex (Kewley \& Ellison 2008).

We have used direct metallicities whenever possible, but the [O III] $4363 \AA$ emission line is faint and rarely observed at high redshift; accordingly, the MEGaSaURA $Z_{\text {neb }}$ values are calculated using the Pettini \& Pagel (2004) $[\mathrm{N} \mathrm{II}] / \mathrm{H} \alpha$ calibration (Table 2 of Rigby et al. 2018a). Meanwhile, optical spectra of the entire low-redshift COS sample are not publicly available, and the literature values have used different strongline calibrations, precluding a uniform metallicity analysis. Of these, the O3N2 method from Pettini \& Pagel (2004), which uses the $([\mathrm{O} \mathrm{III}] 5007 / \mathrm{H} \beta) /([\mathrm{N} \mathrm{II}] 6583 / \mathrm{H} \alpha)$ ratio, is the most common empirical metallicity calibration used for our sample. We have also calculated $12+\log (\mathrm{O} / \mathrm{H})$ using the direct method for three galaxies in the sample following the methods of Berg et al. (2019). Consequently, $12+\log (\mathrm{O} / \mathrm{H})$ (or $Z_{\text {neb }}$ ) is not uniformly calculated and may include systematic calibration uncertainties (Kewley \& Ellison 2008). The low-redshift galaxies span a factor of 50 in $Z_{\text {neb }}$ from 0.03 to $1.5 Z_{\odot}$ (corresponding to $12+\log (\mathrm{O} / \mathrm{H})=7.22-8.87$ ).

All of the galaxies were selected as rest-frame, UV-bright star-forming galaxies such that the sample generally resides above the so-called star-forming main sequence (Figure 1 of Chisholm et al. 2016). Importantly, these rest-frame UV spectra probe a large, and varying, spatial scale. However, each spectrum samples a spatially unresolved stellar population. In other words, each spectrum samples multiple young, UVbright, massive stars. At high redshifts, the physical scale depends on the lensing magnification, which varies from 2 to 200 (Sharon et al. 2019), such that the MEGaSaURA spectra probe multiple star-forming regions within the same galaxy 
(see the analysis in Bordoloi et al. 2016). Similarly, COS is a fixed circular aperture spectrograph with a 2 ". 5 diameter. The low-redshift sample spans a range of $z=0.0007-0.1816$, which corresponds to the COS aperture covering a physical diameter of $50 \mathrm{pc}-10 \mathrm{kpc}$. Many different physical scales (multiple star-forming regions to entire galaxies) reside within the COS aperture.

\section{Stellar Continuum Modeling}

\subsection{Fitting Procedure}

We fit the stellar continua of both the MEGaSaURA and lowredshift samples by assuming that the observed spectra are combinations of multiple bursts of single-age, single-metallicity stellar populations. The light from these stellar populations then propagated through an ambient interstellar medium that attenuated the stellar continuum to produce the observed spectral shape. We fit this with a uniform dust screen model:

$$
F_{\mathrm{obs}}(\lambda)=10^{-0.4 E(B-V) k(\lambda)} \sum_{i} X_{i} M_{i}(\lambda),
$$

where $E(B-V)$ is the stellar attenuation parameter, $k(\lambda)$ is the reddening curve from Reddy et al. (2016), and $X_{i}$ is the linear coefficient multiplied by the $i$ th single-age stellar population model, $M_{i}$. Each $M_{i}$ corresponds to a single-age and singlemetallicity $\left(Z_{*}\right)$ fully theoretical stellar continuum model (see Section 3.2). Thus, $k(\lambda), E(B-V), X_{i}$, and $M_{i}$ completely describe the shape and spectral features of the observed stellar continuum.

We chose the Reddy et al. (2016) attenuation law because it is observationally defined down to $950 \AA$, closer to the ionizing continuum than other models (e.g., Calzetti et al. 2000). This is important because we are predominantly interested in inferring the ionizing continua of massive stars. We tested the effect that changing the attenuation law has on the derived stellar properties and found it to most strongly affect the inferred $E(B-V)$ values, which were $0.01 \mathrm{mag}$ redder, on average, using the Calzetti et al. (2000) law versus the Reddy et al. (2016) law.

We fit the entire observable wavelength regime between 1220 and $2000 \AA$. We did not include wavelengths below $1220 \AA$ due to strong $\operatorname{Ly} \alpha$ features and the Ly $\alpha$ forest. The available rest-frame wavelength regime for each galaxy depends on the observational setup and the redshift of the galaxy. We masked out $\pm 500 \mathrm{~km} \mathrm{~s}^{-1}$ around strong interstellar medium (ISM) absorption and emission lines as well as absorption from foreground systems at lower redshifts (Rigby et al. 2019, in preparation). We optimized this masking velocity interval by studying the individual ISM features at our spectral resolution. One exception is the C IV $1550 \AA$ region, where we only masked out from -500 to $+50 \mathrm{~km} \mathrm{~s}^{-1}$ in order to include the crucial C IV P-Cygni emission (Section 4.1.1). Further, we manually masked out regions that are not stellar continuum features, such as abnormally large ISM absorption, Milky Way features, or sky emission lines. We then fit for the $X_{i}$ and $E(B-V)$ of each single-age, single-metallicity fully theoretical stellar continuum model in Equation (1) using MPFIT (Markwardt 2009).

\subsection{Stellar Models}

The theoretical stellar continuum models $\left(M_{i}(\lambda)\right)$ are key to the spectral population synthesis. We used both single-star models (Leitherer et al. 1999, 2010, 2014) and models that include binary evolution (Eldridge et al. 2017; Stanway \& Eldridge 2018b) to quantify the effect that binary evolution has on the ionizing and non-ionizing continua of massive stars (Section 5.4). We chose the stellar atmosphere models below because they are the most comparable to each other (Eldridge et al. 2017) and have the most observationally motivated massoutflow rates (Leitherer et al. 2010). For both models, we assumed a standard Kroupa IMF (Kroupa 2001) with a broken power law with a high-(low-)mass exponent of 2.3 (1.3) and a high-mass cutoff of $100 M_{\odot}$. Steidel et al. (2016) demonstrated that the high-mass cutoff weakly affected the spectral synthesis fits to FUV stellar continua by showing that the best-fit stellar continua did not change drastically using either a $300 M_{\odot}$ or $100 M_{\odot}$ cutoff (their Figure 7).

Star light between 1200 and $2000 \AA$ is dominated by young, massive O-stars. Consequently, we used fully theoretical stellar models with young ages corresponding to 1, 2, 3, 4, 5, 8, 10, 15, 20, and 40 Myr. At $1270 \AA$, a 20 Myr stellar population of a given initial mass is nearly two orders of magnitude fainter than a 1 Myr stellar population, while older populations are fainter still (Leitherer et al. 1999; Eldridge et al. 2017). Moreover, the UV stellar continua of older stellar populations evolve more slowly with time, such that there are small spectral differences between a 50 and a $100 \mathrm{Myr}$ stellar population (Figure 5 in de Mello et al. 2000). Finally, the effective temperature of B-star populations greater than $40 \mathrm{Myr}$ drops below the $20,000 \mathrm{~K}$ threshold where high-resolution stellar templates are computed using the WM-BASIC code (Leitherer et al. 2010). Thus, the selected age range includes models of the most luminous O-stars whose spectral features vary rapidly with time at sufficient spectral resolution to resolve these important spectral features.

We use the five $Z_{*}$ models that are available from the Geneva stellar atmospheres (Meynet et al. 1994): 0.05, 0.2, 0.4, 1.0 , and $2.0 Z_{\odot}$. Combined, each observed spectrum is fit with 50 fully theoretical stellar models and one free parameter for the dust attenuation, for a total of 51 total free parameters.

Other aspects of stellar physics affect the production of ionizing photons, such as rapid rotation (Levesque et al. 2012; Leitherer et al. 2014; Choi et al. 2017) and a varying (or stochastically populated) IMF (Leitherer et al. 1995; Rigby \& Rieke 2004; Crowther 2007). However, the stellar population synthesis routines only include two $Z_{*}\left(0.14 Z_{\odot}\right.$ and $1 Z_{\odot}$, but see the recent extension to $0.02 Z_{\odot}$ from Groh et al. 2019), which sample metallicity too coarsely for our fitting. Consequently, binary models are the only alternative stellar model that we discuss below.

\subsubsection{The Fiducial Case: STARBURST99 Single-star Models}

We used the fully theoretical STARBURST99 models with Geneva atmospheres that incorporate high mass loss rates (Meynet et al. 1994) as our fiducial model. These models have a spectral resolution of $0.4 \AA$, which matches the spectral resolution of the MEGaSaURA spectra. We convolved the models with a Gaussian to match the observed spectral resolution of each individual MEGaSaURA spectrum, as measured from the optical sky emission lines (Rigby et al. 2018a), and resampled the stellar models onto the wavelength grid of the observations. Similarly, we convolved the higher resolution $H S T / C O S$ data to the $0.4 \AA$ spectral resolution of the STARBURST99 models from the spectral resolution measured from the Milky Way absorption lines (Chisholm et al. 2016). 
Each model spectrum was normalized to the median flux density between 1267 and $1276 \AA$.

The STARBURST99 stellar models were created using the WM-BASIC method (Pauldrach et al. 2001) and densely sample the high-mass portion of the Hertzsprung-Russell diagram up to temperatures of $20,000 \mathrm{~K}$. WM-BASIC does not calculate high-resolution models below these temperatures (Leitherer et al. 2010). Consequently, we chose the 10 stellar ages between 1 and $40 \mathrm{Myr}$ listed above with stellar temperatures greater than $20,000 \mathrm{~K}$. These models include Wolf-Rayet (WR) stars using the Potsdam Wolf-Rayet code (PoWR; Sander et al. 2015), but the evolutionary tracks predict that few if any WR stars are present in a low-metallicity stellar population, such that the WR spectra are rarely incorporated into $0.2-0.4 Z_{\odot}$ STARBURST99 models (Leitherer et al. 2018).

\subsubsection{The Binary Evolution Case: BPASS Models}

We also used the Binary Population and Spectral Synthesis (BPASS) v2.2.1 models, ${ }^{8}$ which include binary star evolution (Stanway \& Eldridge 2018b). BPASS models have a larger metallicity range, but, for consistency, we used the same five $Z_{*}$ available from the Geneva models. BPASS models use a custom set of O-star models created with WM-BASIC at $1 \AA$ resolution for O-stars with temperatures greater than $25,000 \mathrm{~K}$ (Eldridge et al. 2017). Temperatures less than this have the BASELv3.1 and C3K models, which have spectral resolution of $20 \AA$ below $1500 \AA$ (Westera et al. 2002; Le Borgne et al. 2003; Conroy \& van Dokkum 2012; Conroy et al. 2014). Therefore, BPASS models are lower resolution when ages are greater than $20 \mathrm{Myr}$ for any metallicity and when ages are greater than $15 \mathrm{Myr}$ for metallicities greater than $0.4 Z_{\odot}$ (see Figure 1). This spectral resolution is too low to reveal many of the narrow B-star features of older stellar populations and cannot be used to distinguish older stellar populations. For this reason, we chose the STARBURST99 models as our fiducial model. We return to this issue in Section 5.4.2.

\subsection{The Nebular Continuum}

Young, massive stars produce large amounts of ionizing photons, which produce free-free, free-bound, and two-photon nebular continuum emission. The nebular continuum heavily contributes to the total continuum flux at young ages, low metallicities, and redder wavelengths (Steidel et al. 2016; Byler et al. 2018). For a stellar continuum metallicity of $0.05 Z_{\odot}$ and a stellar age of $1 \mathrm{Myr}$, the nebular continuum is $25 \%$ of the stellar continuum at $2000 \AA$.

We created a nebular continuum for each age, metallicity, and stellar model by processing the stellar continuum models through CLOUDY v17.0 (Ferland et al. 2013). We assumed that the gas-phase metallicity and stellar metallicity were the same (Section 5.1), an ionization parameter of $\log (U)=-2.5$, and $n_{\mathrm{H}}=100 \mathrm{~cm}^{-3}$. We produced a nebular continuum for each stellar population, added the output nebular continua to the stellar models, and normalized by the flux between 1267 and $1276 \AA$. The inclusion of the nebular continuum produces redder stellar models than before, which has a pronounced effect on the fitted $E(B-V)$ of young stellar populations.

We tested the effect that different ionization parameters have on the fitted stellar ages and metallicities by also creating

\footnotetext{
https://flexiblelearning.auckland.ac.nz/bpass/9.html
}

models with $\log (U)=-2,-2.3,-2.7$, and -3.0 . We found that the reduced $\chi^{2}$ values of the resultant fits do not change statistically for the different $\log (U)$ values. Consequently, we adopted a midrange $\log (U)=-2.5$ for all galaxies.

\subsection{Stellar Population Parameters Derived from the Fits}

The observed stellar continua are fit by statistically determining the linear multiplicative coefficient, $X_{i}$, for the 50 single-age, single-metallicity stellar models $\left(M_{i}\right.$; Equation (1)). These linear coefficients can take any value greater than or equal to zero, and MPFIT determines the linear combination that best fits the observed stellar continuum. In practice, the code typically assigns $X_{i}=0$ values to most of the stellar models and only gives power to a small subset of the models (on average, six models have light fractions $>0 \%$ ). Figure 2 shows the distribution of the light at a given age for two observed stellar populations fitted with the multiple-population method. All of the light from RCS Knot E comes from a very young, moderate-metallicity stellar population (left panel); while the stellar light from S1527+0652 is broadly distributed across age and metallicity (right panel).

We determined intrinsic stellar parameters from the fitted coefficients in Equation (1). The derived parameters are a weighted average of the total light at $1270 \AA$ attributed to the individual stellar models $\left(X_{i}\right)$. Thus, each property derived below is a "light-weighted" property. First, the light fraction $\left(f_{i}\right)$ that each model contributes to the total intrinsic flux at $1270 \AA$ is defined as

$$
f_{i}=\frac{X_{i}}{\sum_{i} X_{i}}
$$

Second, the light-weighted age at $1270 \AA$ is defined as

$$
\text { Age }=\frac{\Sigma_{i} X_{i} \mathrm{Age}_{i}}{\Sigma_{i} X_{i}}
$$

The light-weighted ages of RCS Knot E and S1527+0652 are indicated as vertical lines in the left panel of Figure 2. Finally, we computed the light-weighted stellar metallicity as

$$
Z_{s}=\frac{\Sigma_{i} X_{i} Z_{i}}{\Sigma_{i} X_{i}}
$$

These three parameters describe the properties of the observed stellar populations. The uncertainties on these parameters were derived by varying the observed flux density at every wavelength by a random Gaussian kernel with width equal to the flux uncertainty at that wavelength. We then recalculated the ages and $Z_{*}$, tabulated each value, and repeated the procedure 100 times. The standard deviation of each age and $Z_{*}$ distribution is the uncertainty on the age and $Z_{*}$, respectively. We include the $f_{i}, Z_{*}$, and light-weighted ages in the electronic version of the Appendix.

All of these stellar population properties (age and metallicity) are light-weighted at $1270 \AA$; they cannot be directly compared to similar properties derived at other wavelengths. Younger stars produce relatively more light at bluer wavelengths than older stars, biasing the light from young stars to bluer wavelengths. The ages derived from full spectral energy distribution (SED) modeling using optical and near-infrared observations will inherently return older ages than we estimated because optical light comes from older stars. 


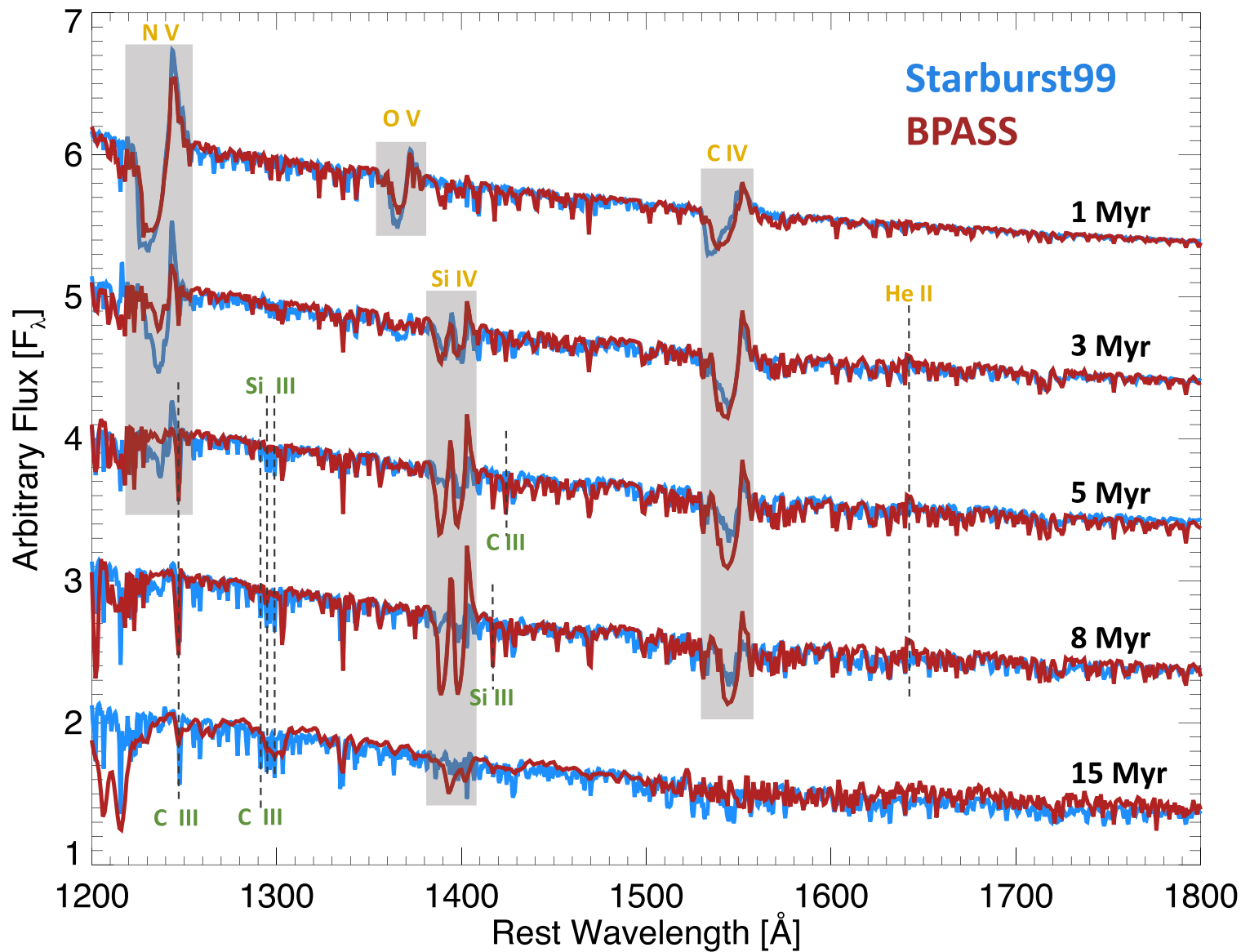

Figure 1. Fully theoretical rest-frame FUV stellar continuum of five STARBURST99 (blue) and BPASS (red) single-burst models with a metallicity of $0.4 Z_{\odot}$. Each spectrum shows a different age, with age decreasing from $1 \mathrm{Myr}$ at the top to $15 \mathrm{Myr}$ at the bottom (labeled on the right). Prominent stellar wind (orange) and photospheric (green) lines are labeled near the models where the features are strongest. The displayed models are a subset of the 50 stellar continuum models that determine the stellar population properties and demonstrate the spectral variations in the rest-frame FUV with stellar population age.

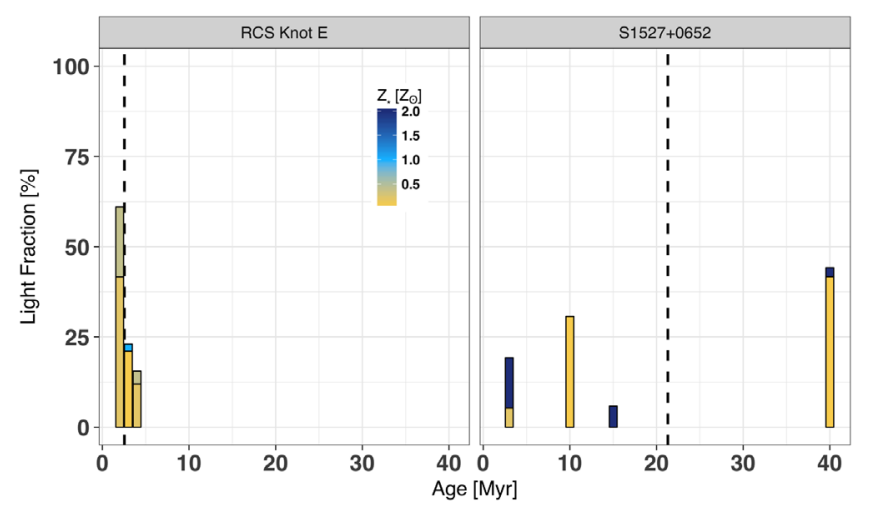

Figure 2. Comparison of the fitted light fractions for each stellar age used in Equation (1) to determine the fit to the FUV spectra for two galaxies: RCS Knot E (left panel) and S1527+0652 (right panel). The total light-weighted ages are given as the dashed vertical lines. RCS Knot E has a very young lightweighted age, 2.5 Myr, with all of the light coming from three ages: 2, 3, and 4 Myr. S1527+0652 has an older light-weighted age, and the fitted models have a mix of old and young populations. Each bar is color coded by the stellar metallicity of the corresponding model. Note that the model grid is not evenly spaced, such that there are not models between each age. For instance, models do not exist between 10 and $15 \mathrm{Myr}$, between 20 and $40 \mathrm{Myr}$, or beyond $40 \mathrm{Myr}$.

An important measure of the ionizing continuum is the ratio of the intrinsic flux density at $900 \AA$ to the flux density at $1500 \AA\left(F_{900} / F_{1500}\right)$. Observations compare the observed
$F_{900} / F_{1500}$ to the intrinsic $F_{900} / F_{1500}$ to determine the fraction of ionizing photons that escape galaxies (Steidel et al. 2001). We estimate the intrinsic $F_{900} / F_{1500}$ by extending the stellar population fits to bluer wavelengths than the observations and removing the contributions from dust attenuation (setting $E(B-V)=0$ in Equation (1)). The high-resolution STARBURST99 models are only defined at $>905 \AA$, so we created low-resolution STARBURST99 models $(20 \AA$ resolution; Leitherer et al. 1999) using the same model parameters and fitted light fractions as the high-resolution models. We then measured the median model flux density between 895 and $906 \AA$ for $F_{900}$, and $1495-1506 \AA$ for $F_{1500}$.

Ionizing photons with higher energies create high-ionization gas (e.g., $\mathrm{O}^{++}$). We also inferred the stellar flux density between 510 and $540 \AA\left(F_{525}\right)$ and between 280 and $320 \AA$ $\left(F_{300}\right)$ to determine how many high-energy photons a given stellar population produces. The flux density $F_{525}$ (with photon energies of $24 \mathrm{eV}$ ) probes photons that singly ionize oxygen but do not ionize helium. Meanwhile, $F_{300}$ (photon energies of $41 \mathrm{eV}$ ) probes photons that doubly ionize oxygen and singly ionize helium, but do not doubly ionize helium. These wavelengths were carefully chosen to probe the peak of the stellar SEDs, while avoiding contributions from strong stellar absorption and emission features (see Figure 1).

Finally, all derived parameters are either flux density ratios (e.g., $\left.F_{900} / F_{1500}\right)$ or derived from normalized spectra (e.g., stellar age). This means that the stellar population parameters 
Table 1

Prominent Stellar Features

\begin{tabular}{lcc}
\hline \hline Line & $\begin{array}{c}\text { BPASS Age } \\
\text { (Myr) }\end{array}$ & $\begin{array}{c}\text { STARBURST99 Age } \\
\text { (Myr) }\end{array}$ \\
\hline & Stellar wind lines & \\
\hline N V 1240 & $1-5$ & $1-10$ \\
O V 1371 & $1-2$ & $1-4$ \\
Si IV 1400 & $3-15$ & $3-5$ \\
C IV 1550 & $1-15$ & $1-10$ \\
He II 1640 & $4-20$ & $3-4$ \\
\hline & Photospheric lines & $5-40$ \\
\hline C III 1247.4 & $4-15$ & $5-40$ \\
Si III 1294.5 & $\ldots$ & $\ldots$ \\
C III 1296.3 & $\ldots$ & $5-40$ \\
Si III 1296.7 & $\ldots$ & $5-40$ \\
Si III 1298.9 & $\ldots$ & $1-20$ \\
Fe V 1346-1365 & $1-15$ & $1-20$ \\
Fe V 1427-1430 & $1-15$ & $1-40$ \\
S V 1501.8 & $1-8$ & $3-40$ \\
Fe IV 1526-1534 & $3-10$ & $8-40$ \\
Si II 1533 & $\ldots-40$ & $10-40$ \\
Fe III 1923-1966 & $10-4$ & \\
\hline
\end{tabular}

Note. The first column gives the feature and wavelength. The second and third columns give the age range that the features are found in, in a $0.4 Z_{\odot}$ BPASS and STARBURST99 model, respectively.

are independent of the intrinsic luminosity, which depends on the magnification from gravitational lensing.

\section{Relating Spectral Features and Inferred Stellar Population Properties}

Oftentimes, the ages of stellar populations are deduced using the broadband UV through infrared (IR) SED shapes. While the SED shape provides important age information, it is often degenerate with metallicity and dust attenuation. By contrast, fitting the stellar spectral features with theoretical stellar templates simultaneously determines the age, metallicity, and dust attenuation of the stellar populations. Consequently, the light-weighted ages and metallicities derived above are driven by spectral features that are less degenerate than the spectral shape alone (see Figure 1 and Table 1).

The two main types of FUV stellar features are strong, broad stellar wind P-Cygni features and weak stellar photospheric absorption lines. Both types of features can be contaminated by neighboring ISM absorption and require high $\mathrm{S} / \mathrm{N}$ and moderate spectral resolutions to resolve. In the following two subsections, we discuss both types of spectral features individually, and we illustrate how the individual features relate to the inferred stellar population ages and metallicities. The purpose of these subsections is not to advocate for determining the stellar ages and metallicities using single features, but rather to demonstrate that the stellar properties inferred from the full spectral fits are entirely consistent with the trends of stellar spectral features.

\subsection{Stellar Wind Features}

The most notable stellar features in the FUV are the broad blueshifted absorption and redshifted emission profiles (called P-Cygni profiles; orange labels in Figure 1). These P-Cygni profiles arise from strong winds that are radiatively driven off of stellar photospheres (Castor et al. 1975; Lamers \& Cassinelli 1999). The terminal velocity, ionization structure, and mass-outflow rates sensitively depend on the stellar luminosity (Castor et al. 1975; Lamers \& Leitherer 1993; Lamers et al. 1995; Leitherer et al. 1995; Puls et al. 1996; Kudritzki \& Puls 2000). The terminal velocity describes the maximal velocity extent of the absorption component, while the mass-loss rate determines the depth of the profile. In turn, these establish the shape of the P-Cygni absorption and emission.

Whether a given ion is observed as a P-Cygni profile in the wind depends on the ionization structure of the stellar wind. The peak ionization stages for stellar winds typically are the C V, N IV, O IV, and Si V states (Lamers \& Cassinelli 1999), none of which have resonant transitions in the rest-frame FUV. Alternatively, the presence of adjacent ionization stages with P-Cygni profiles (N V, C IV, or Si IV) provides information on the stellar temperature of the most luminous stars and, by inference, the stellar population age. The age of a stellar population can be inferred from the strength of the observed P-Cygni transitions: $\mathrm{OV}$ and $\mathrm{N} \mathrm{V}$ have the highest ionization states and are strongest in stars with lifetimes of 2-3 Myr, while C IV and Si IV are lower ionization and peak in stars with lifetimes near $5 \mathrm{Myr}$.

Stellar temperature, or age, is not the sole determinant of the stellar wind profiles. Metals in the photospheres of hot stars absorb continuum photons, which is what accelerates the gas off the stellar surface. Consequently, the stellar metallicity determines both the acceleration and mass-outflow rate of the stellar wind (Lamers \& Cassinelli 1999; Vink et al. 2001). The terminal velocities and mass-loss rates of O-stars with $>0.2 \mathrm{Z}$. have been empirically determined to scale as $Z_{*}^{0.13}$ and $Z_{*}^{0.69}$, respectively (Leitherer et al. 1992; Vink et al. 2001). Lowermetallicity stellar winds of resolved individual stars have not been observed, so the mass-outflow rate and terminal velocity relations have been extrapolated to lower metallicities. These relations illustrate how stellar wind P-Cygni absorption profiles scale with stellar metallicity.

In the next five subsections, we walk through the individual P-Cygni lines in the FUV. Each subsection explores the theoretical and observed wind features and their relationship to the inferred stellar population age and metallicity. In Section 4.3 we conclude that the N V P-Cygni and He II emission are strong in very young stellar populations, while the shape of the C IV P-Cygni profile changes both with stellar age and metallicity (see Figure 3). Conversely, the Si IV of our sample is dominated by interstellar absorption, and the $\mathrm{OV}$ line is not observed. Collectively, the stellar wind profiles mimic the inferred stellar ages and metallicities.

\subsubsection{The CIV P-Cygni Feature}

The CIV feature is strong and broad and has a P-Cygni profile for all of the MEGaSaURA galaxies (there is no C IV coverage for many of the low-redshift COS spectra). Further, the CIV P-Cygni profile is sufficiently broad that stellar and interstellar components can easily be separated with moderate spectral resolution (see shaded regions in Figures 4 and 5). The CIV profile probes stellar outflows from 1 to $10 \mathrm{Myr}$ populations, and the absorption component distinctly varies with $Z_{*}$ (Figure 3 ). This makes it an ideal diagnostic of stellar age and metallicity. 


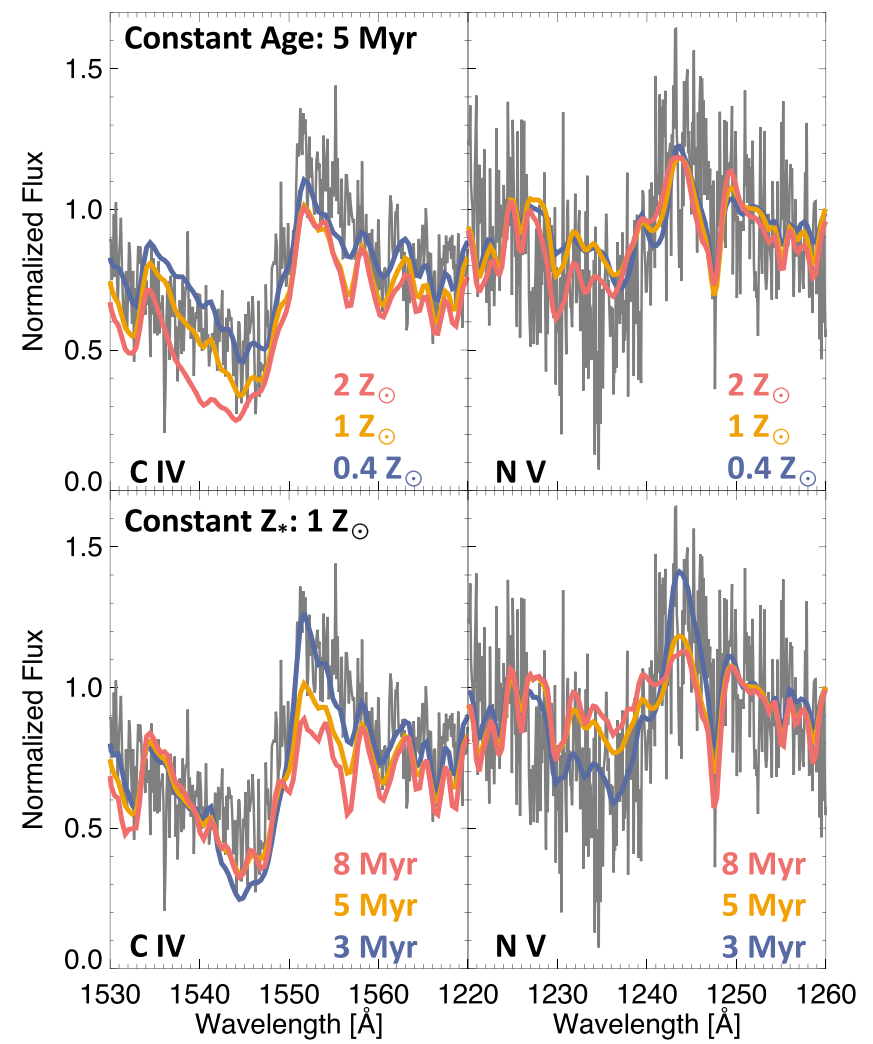

Figure 3. Dependence of the C IV $1550 \AA$ (left panels) and N V $1240 \AA$ (right panels) stellar wind profiles on stellar age and metallicity. The observed stellar wind profile from $\mathrm{S} 0033+0242$ is shown in gray in each panel. In the upper panels, we overlay three STARBURST99 theoretical models with a constant 5 Myr age but with a varying stellar metallicity $\left(Z_{*}\right)$ of 2 (red), 1 (gold), and $0.4 Z_{\odot}$ (blue). The bottom two panels show three STARBURST99 models with a constant $Z_{*}=1 Z_{\odot}$ but with varying stellar age of 8 (red), 5 (gold), and $3 \mathrm{Myr}$ (blue). $Z_{*}$ does not change the $\mathrm{N} v$ profile (upper right panel), but the emission profile strongly peaks for ages $<5 \mathrm{Myr}$ (lower right panel). $Z_{*}$ strongly impacts the C IV absorption (wavelengths less than $1550 \AA$ ), but weakly impacts the C IV emission (upper left panel). Conversely, the stellar age strongly impacts the C IV emission (wavelengths greater than $1550 \AA$ ) but weakly impacts the C IV absorption (lower left panel). By eye, the C IV and N V profiles of S0033 +0242 are best fit between the blue and gold profiles in each panel. This is consistent with the light-weighted age and metallicity of $5 \pm 0.5 \mathrm{Myr}$ and $0.84 \pm 0.04 Z_{\odot}$

Different portions of the C IV profile depend on either $Z_{*}$ or stellar age (left panels of Figure 3). At a constant $5 \mathrm{Myr}$ age (upper left panel), the C IV absorption of the STARBURST99 models deepens and broadens as $Z_{*}$ increases from $0.4 Z_{\odot}$ (blue line) to $1 Z_{\odot}$ (gold line) to $2 Z_{\odot}$ (pink line), because the C IV mass-loss rate strongly increases with $Z_{*}$. Conversely, the models predict that the C IV emission is nearly independent of $Z_{*}$. Meanwhile, at a constant $1 Z_{\odot}$ metallicity (lower left panel), the modeled C IV absorption is independent of stellar age, but the emission strongly peaks at ages $<5 \mathrm{Myr}$ (blue line). In summary, the $\mathrm{C}$ IV absorption varies with $Z_{*}$ and the C IV emission varies with stellar age.

The temporal evolution of C IV found in the models is clearly corroborated by the light-weighted ages. Figure 4 shows three MEGaSaURA spectra ordered in descending lightweighted stellar age, and each have similar light-weighted metallicities $\left(0.29 \pm 0.06 Z_{\odot}\right)$. Overplotted in red is a singleage STARBURST99 model with a population age nearest to the inferred light-weighted age. The C IV emission is strongest in

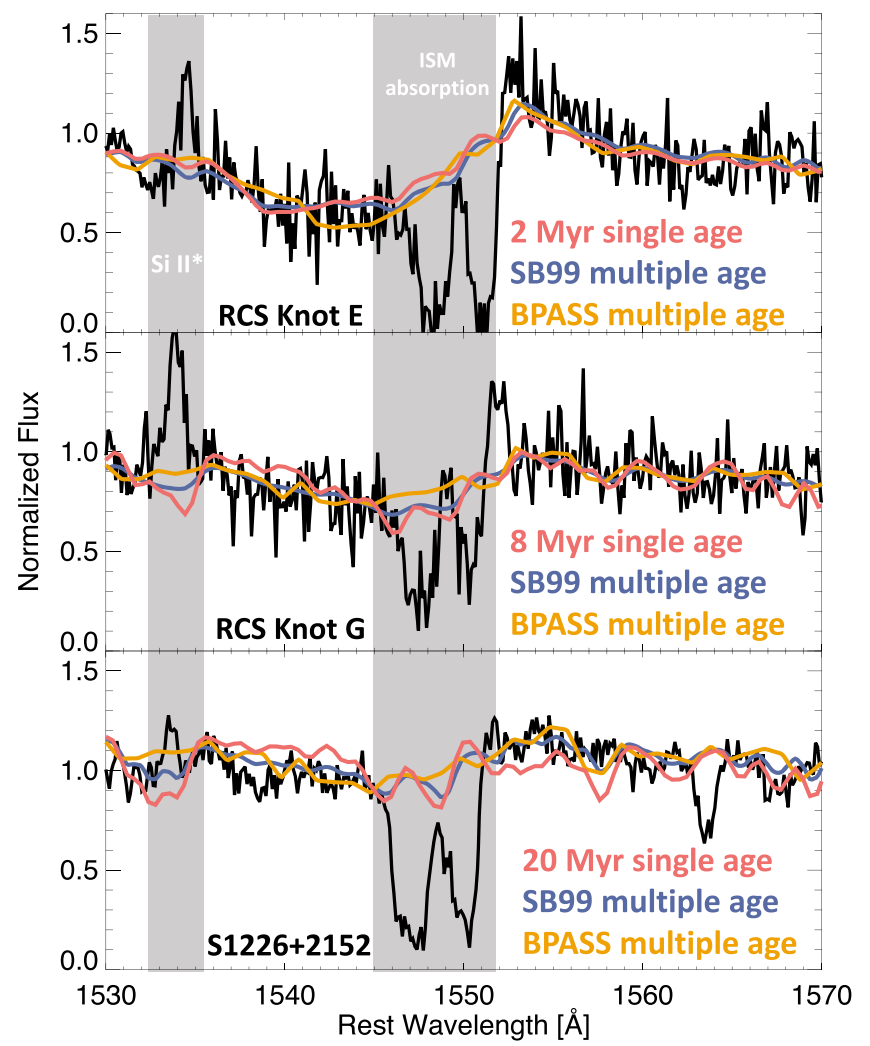

Figure 4. Dependence of the C IV wind profile on stellar age. Each panel shows an observed galaxy spectrum (black line) ordered by descending fitted light-weighted ages of $2.5 \pm 0.1,11 \pm 1.8$, and $26 \pm 1.3 \mathrm{Myr}$. The fitted metallicities are similar $\left(0.29 \pm 0.04 Z_{\odot}\right)$. The colored curves are theoretical models. A single-age, $0.2 Z_{\odot}$ STARBURST99 theoretical model with an age nearest to the fitted light-weighted age is overplotted in red. The multiple-age, multiple-metallicity BPASS (blue) and STARBURST99 (gold) fits are also shown. The single-age models describe the overall shape of the younger $\mathrm{C}$ IV profiles (e.g., RCS Knot E and G), while the multiple-age fits are required to capture the full details of the older profiles (e.g., S1226+2152). Gray regions correspond to interstellar C IV absorption and Si II* emission.

the youngest populations and adequately matches the triangular wind emission from the RCS Knot E spectrum. Meanwhile, RCS Knot G, a different region from the same galaxy, has weaker P-Cygni emission (although note the narrow nebular C IV emission), similar to an $8 \mathrm{Myr}$ single-age population. Finally, S1226+2152 has an even older inferred light-weighted age; the C IV P-Cygni feature has nearly disappeared and has been replaced by a flat C IV feature with narrow interstellar absorption. The derived light-weighted ages of each spectrum are consistent with the by-eye P-Cygni variation: Knot E, Knot G, and S1226+2152 have STARBURST99 light-weighted ages of $2.5,11$, and $26 \mathrm{Myr}$, respectively.

While the single-age models reflect the C IV profiles of the youngest populations, they do not describe all of the galaxy-togalaxy P-Cygni variations in the older populations. The C IV absorption from RCS Knot G is deeper at bluer velocities than the $8 \mathrm{Myr}$ single-age model, while S1226+2152 has a small amount of redshifted emission. The observed stellar populations are not single-age populations, but the multiple-age fits capture the different populations (blue and gold lines in Figure 4 are the STARBURST 99 and BPASS fits). While a singleage population largely describes the C IV emission from the youngest populations, older populations require a mix of both young and old stars to match the observations. 


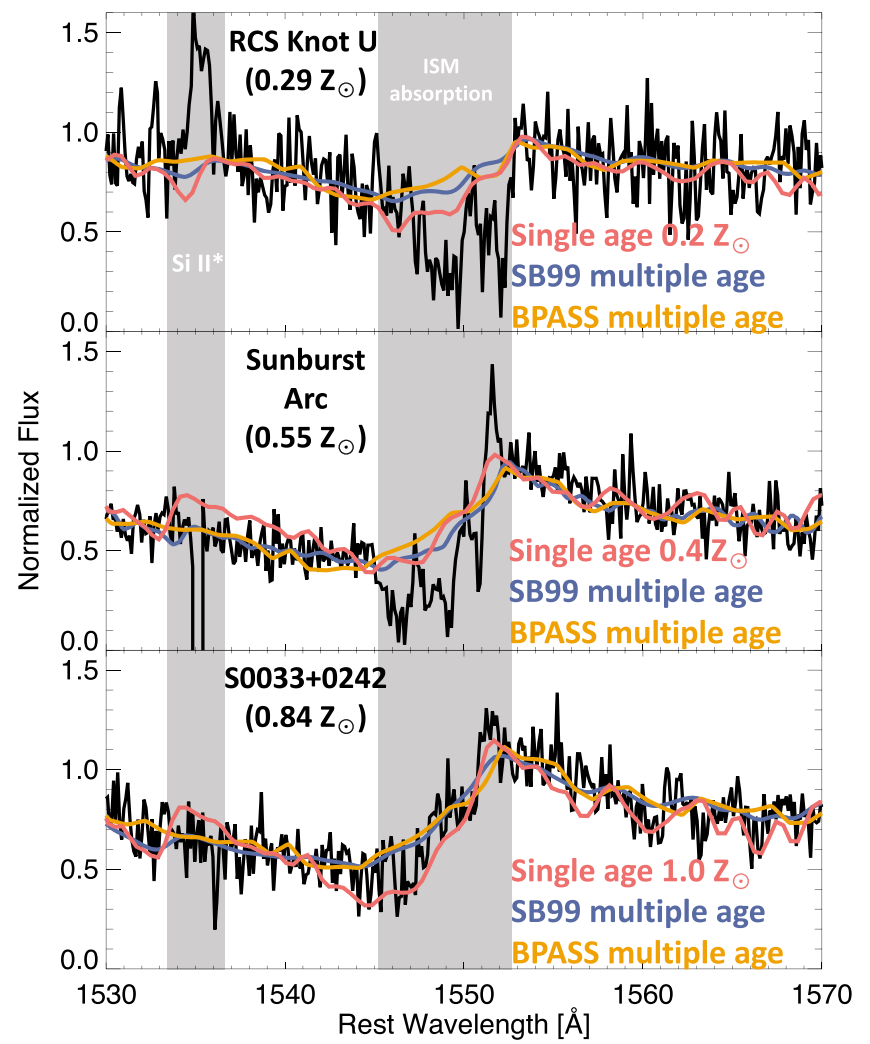

Figure 5. Dependence of the C IV stellar wind profile on stellar metallicity $\left(Z_{*}\right)$ at nearly constant stellar age $(4 \pm 1 \mathrm{Myr})$. The three panels show the observed spectra (in black) ordered by descending fitted $Z_{*}$ of $0.29 \pm 0.07,0.55 \pm 0.04$ and $0.84 \pm 0.04 Z_{\odot}$. The C IV stellar absorption increases with increasing $Z_{*}$. The colored curves correspond to various models. A 5 Myr STARBURST99 model is shown in red in each panel with $Z_{*}$ closest to the inferred lightweighted $Z_{*}$. The multiple-age, multiple-metallicity BPASS (blue) and STARBURST99 (gold) fits are also shown. At these young ages, the singleage models describe the overall shape of the C IV lines. Gray regions correspond to interstellar C IV absorption and Si II* emission.

Metallicity has a similarly strong effect on the shape of the observed C IV profile, but this time on the absorption portion of the P-Cygni profile. The upper left panel of Figure 3 shows that $Z_{*}$ mostly affects the depth and width of the absorption component. This is further illustrated in Figure 5, which shows three MEGaSaURA spectra with nearly constant stellar age (5, 3, and $5 \mathrm{Myr}$ ) but with increasing stellar metallicity. At $0.3 Z_{\odot}$ (top panel), the C IV absorption only reaches a depth of 0.6 in normalized flux units. Increasing the metallicity to $0.6 Z_{\odot}$ (middle panel) creates a pronounced, broad P-Cygni absorption profile that reaches 0.5 in normalized flux units. Finally, by $0.8 Z_{\odot} \quad$ (bottom panel), the stellar wind dominates the C IV spectral region, and the absorption reaches 0.4 in normalized flux units. The stellar emission does not strongly change with $Z_{*}$, as the stellar emission peaks near $1.05 \pm 0.05$ for all of these spectra. Both the stellar models and the observed spectra indicate that the stellar metallicity strongly shapes the absorption component of the C IV P-Cygni profile.

To summarize: the C IV profile strongly varies with the inferred light-weighted stellar population properties. The C IV P-Cygni absorption depends on the $Z_{*}$, and the emission depends on the stellar age (Figure 3). The multiple-age and multiple-metallicity fits to the CIV P-Cygni profiles mimic changes in the inferred stellar age (Figure 4) and metallicity (Figure 5).

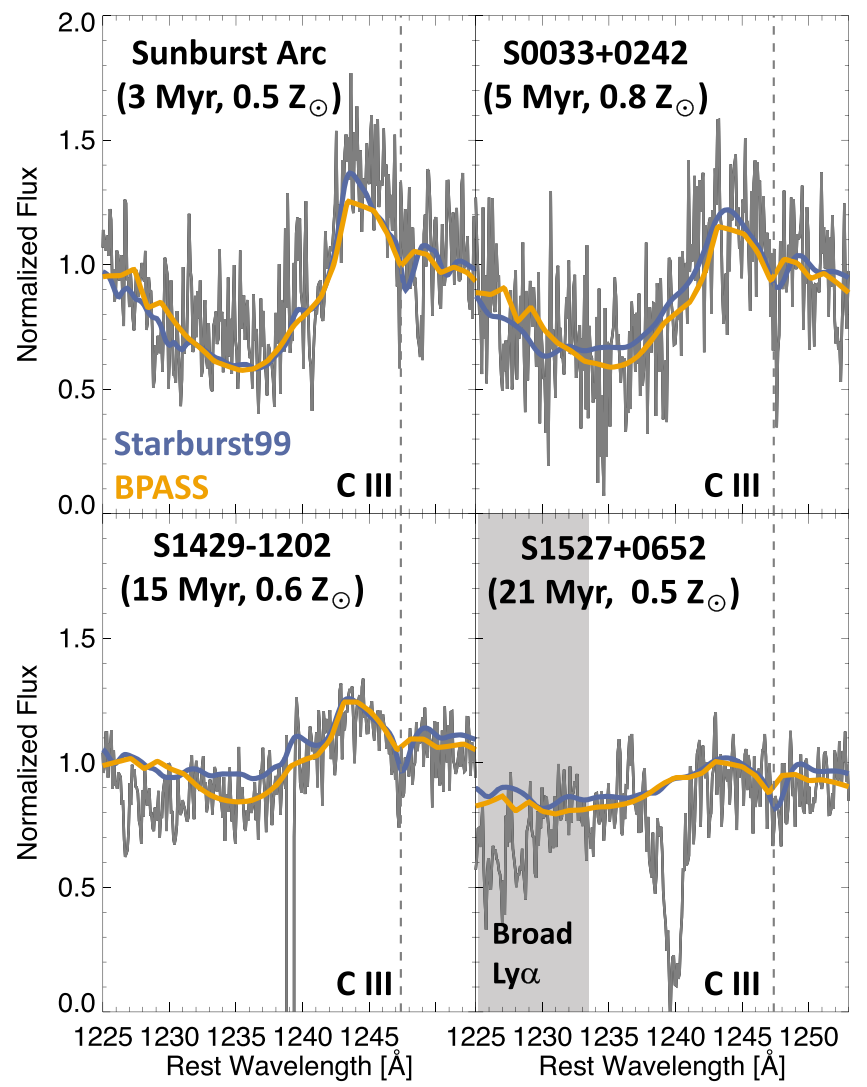

Figure 6. Observed N V $1240 \AA$ stellar wind profiles of four galaxies in gray. The panels are arranged from youngest stellar age in the upper left to oldest in the lower right. The $\mathrm{N} \mathrm{V}$ wind profile transitions from a strong P-Cygni profile in the upper left to a nearly flat continuum in the lower right as the lightweighted age declines. The four stellar populations have similar $Z_{*}$ but different light-weighted ages, emphasizing that the N V line chiefly depends on stellar age (see the right panel of Figure 3). The STARBURST99 (blue) and BPASS (gold) fits are included in each panel. The dashed gray line denotes the C III $1247 \AA$ photospheric line.

\subsubsection{The NV P-Cygni Feature}

The N V $1240 \AA$ A stellar wind profile largely depends only on stellar age. The age dependence arises because the dominant ionization state of an O-star wind is $\mathrm{N}^{+++}$, but as the stellar temperature increases with decreasing $Z_{*}, \mathrm{~N}^{+++}$gas is heated into the $\mathrm{N}^{4+}$ state. This heating produces relatively more gas in the $\mathrm{N}^{4+}$ ionization state for low- $Z_{*}$ winds than higher- $Z_{*}$ winds and nearly balances the decreasing metallicity (Kudritzki 1998; Lamers \& Cassinelli 1999; Leitherer et al. 2010). The models in the upper right panel of Figure 3 show the negligible $\mathrm{N} \mathrm{V}$ variation with $Z_{*}$, while strong absorption and emission only occur at ages young enough to produce $\mathrm{NV}$ stellar winds (bottom right panel of Figure 3). N V only arises from very young $(<5 \mathrm{Myr})$ stellar populations.

A strong $\mathrm{N} V$ profile is detected in most FUV spectra in Figure 6. The exceptions are the oldest stellar populations, which do not show P-Cygni profiles in either the C IV or the $\mathrm{NV}$ ionization state (e.g., S1527+0652). Weak C IV and a nondetection of $\mathrm{N} \mathrm{V}$ indicate that there is not currently a young $(<8 \mathrm{Myr})$ stellar population in these older galaxies (see the discussion in Section 5.3). In contrast, the normalized flux of the N V profile from the Sunburst Arc varies by a factor of 2.5 from the absorption depth to the emission peak, illustrating the strong N V P-Cygni profiles in populations with very young light-weighted ages. This trough-to-peak ratio decreases with 


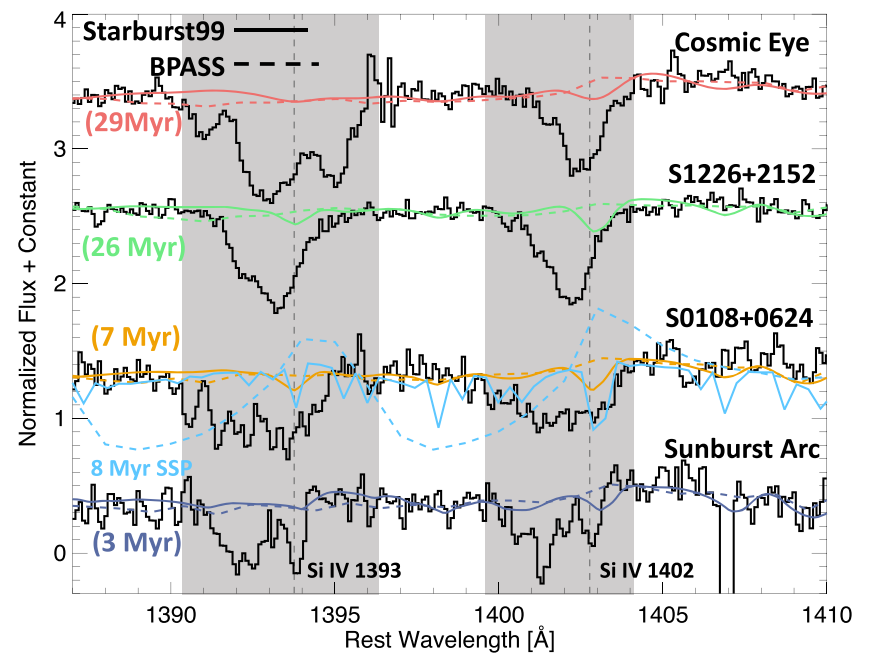

Figure 7. Si IV $1400 \AA$ stellar wind region for four galaxies ordered by ascending light-weighted age. The multiple-age and multiple-metallicity STARBURST99 (solid line) and BPASS (dashed line) fits are overplotted for each galaxy. Areas of strong ISM absorption, which dominates the Si IV spectral regime, are shaded in gray. An $8 \mathrm{Myr}$ single-age model is included over the S0108+0624 spectra in light blue to demonstrate that BPASS models predict strong P-Cygni profiles at these ages, while the STARBURST99 models do not.

increasing inferred stellar population age in Figure 6: it is 2.2 for S0033+0242 with an age of $5 \mathrm{Myr}$ and 1.4 for S1429-1202 with an age of $15 \mathrm{Myr}$.

\subsubsection{The Si IV P-Cygni Feature}

P-Cygni Si IV is generally observed in supergiants (evolved stars) or in metal-rich stars because the dominant ionization state in main-sequence stellar winds is the $\mathrm{Si}^{4+}$ state. Thus, the $\mathrm{Si}^{+++}$ionization state is too low of a temperature to trace the bulk of the O-star wind (the opposite behavior as $\mathrm{N} \mathrm{V}$ above; Walborn \& Panek 1984). As O-stars evolve into supergiants, their stellar photospheres expand and the stellar winds become denser, causing the dominant ionization state, $\mathrm{Si}^{4+}$, to recombine into $\mathrm{Si}^{+++}$. These denser winds produce prominent Si IV P-Cygni features in the spectra of evolved O-stars (Drew 1989; Pauldrach et al. 1990). Similarly, the ionization structure also shifts toward lower ionization stages as larger stellar metallicities produce relatively more $\mathrm{Si}^{+++}$in their stellar winds. Thus, the stellar models predict that Si IV P-Cygni profiles are only strong in stellar populations with a lower ionization structure, either from higher metallicity stars or an outsized contribution of evolved stars.

Strong Si IV $1400 \AA$ A ISM absorption is seen in Figure 7, but $\mathrm{Si}$ IV is rarely observed to have a stellar P-Cygni profile. The entirety of the observed Si IV absorption can be explained by a narrow interstellar absorption that reaches nearly zero flux. After accounting for interstellar absorption (gray regions in Figure 7), the observed Si IV region is nearly featureless. The lack of strong P-Cygni Si IV suggests that the stellar populations within our sample are either metal-poor (see Section 5.1) or not dominated by evolved stars. We conclude that the observed Si IV regions are dominated by interstellar absorption and that Si IV does not strongly vary with the stellar population properties.

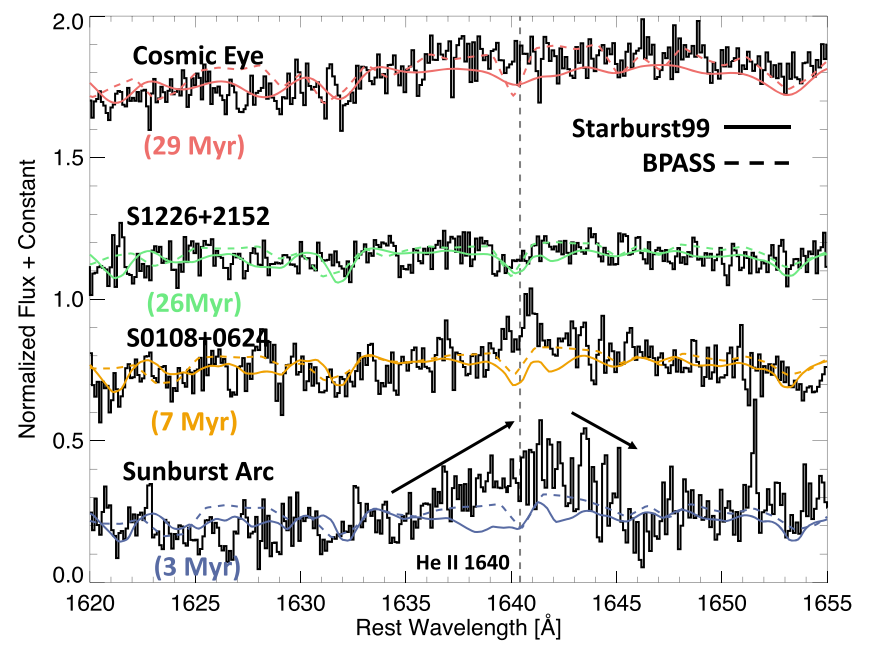

Figure 8. Observed He II $1640 \AA$ A region for four MEGaSaURA spectra in black. Overplotted on each is the STARBURST99 (solid line) and BPASS (dashed line) multiple-age stellar population fit. Generally, the BPASS models fit the He II region better than the STARBURST99 models. However, neither model adequately fits J0108+0624 or the Sunburst Arc (bottom two spectra). J0108 +0624 has narrow emission $\left(96 \mathrm{~km} \mathrm{~s}^{-1}\right)$ that is likely nebular in origin, while the Sunburst Arc has broad emission $\left(379 \mathrm{~km} \mathrm{~s}^{-1}\right)$ that is likely stellar in origin. The arrows above the Sunburst Arc's spectrum emphasize the broad emission feature. The other young populations have similarly broad He II emission.

\subsubsection{The He II Emission Feature}

He II $1640 \AA$ stellar emission arises from extremely hot, evolved WR stars. WR stars are a short-lived supergiant phase associated with stars that have main-sequence lifetimes less than 5 Myr (Abbott \& Conti 1987; Crowther 2007). WR stars have broad He II emission lines in both the optical and FUV that are strongest in higher metallicity stars (Schaerer \& Vacca 1998).

He II in Figure 8 is not observed as a broad P-Cygni profile like N V or C IV. For the oldest light-weighted populations, He II is a weak absorption line (equivalent width of $0.13 \pm 0.04 \AA$ in $\mathrm{S} 1226+2152$ ). Conversely, the youngest populations (Sunburst Arc, RCS Knot E, S0033+0242, and RCS Knot U) have a broad triangular He II emission profile (see the arrows in Figure 8). The He II emission resembles the redshifted triangular C IV emission profile but without the blueshifted absorption component that creates the P-Cygni profile. There is no He II absorption because He II $1640 \AA$ is not a resonant transition (it is analogous to $\mathrm{H} \alpha$ ), so the stellar wind is optically thin to the He II recombination emission. The He II emission equivalent width from the Sunburst Arc spectrum is $-1.2 \AA$, and it has an FWHM $=379 \mathrm{~km} \mathrm{~s}^{-1}$, consistent with a $3 \mathrm{Myr}, 0.5 Z_{\odot}$ WR model (Schaerer \& Vacca 1998). The presence of a broad He II emission profile strongly suggests that the spectrum is dominated by a $<5 \mathrm{Myr}$ stellar population.

Finally, S0108+0624 is the only galaxy in the sample with statistically significant $(>3 \sigma)$, narrow He II emission (equivalent width of $-0.33 \pm 0.08 \AA$ and FWHM $=96 \mathrm{~km} \mathrm{~s}^{-1}$, which is resolved by the $69 \mathrm{~km} \mathrm{~s}^{-1}$ spectral resolution). This narrow emission appears nebular in origin when compared to the broad WR feature of the Sunburst Arc (compare the bottom and second-to-bottom spectra in Figure 8). This He II emission is at the weak end of the range of He II equivalent widths seen in local dwarf galaxies ( -0.4 to $-3.4 \AA$; Berg et al. 2016, 2019). 
We return to possible origins of the nebular He II emission in Section 5.4.4.

The multiple-age fits to the He II region are fairly poor. This is especially true for galaxies with strong WR emission (e.g., the Sunburst Arc). The BPASS models fit the He II region substantially better than the STARBURST99 models do, although they still do not match the WR features of younger populations. While both stellar models include WR models, neither model appears to produce a sufficient number of WR stars to match the broad He II emission observed in the youngest stellar populations (e.g., Leitherer et al. 2018).

Overall, we find broad WR He II emission in stellar populations with the youngest light-weighted ages and weak He II absorption in older populations.

\subsubsection{The $O V$ Wind Feature}

With an ionization potential of $117 \mathrm{eV}, \mathrm{OV} 1371 \AA$ is the highest ionization line between 1200 and $2000 \AA$ and traces the hottest outflowing phase from the most massive stars (up to $\left.10^{6} \mathrm{~K}\right)$. This feature is an unambiguous indicator of the youngest stars and disappears once the stellar population ages past 3 Myr (see Figure 1 and Table 1).

While $\mathrm{OV}$ is an ideal tracer of the most massive stars, it is not strongly observed in any of the MEGaSaURA or COS spectra. This may indicate that there is not a significant extremely massive stellar population $\left(\sim 300 M_{\odot}\right)$, but more likely it indicates that the winds of the most massive stars are significantly clumpier-thus denser-than assumed in the model atmospheres. Denser, clumpier winds lead to lower ionization stellar winds and reduced $\mathrm{O} \mathrm{V}$ profiles (Bouret et al. 2003; Leitherer et al. 2010).

\subsection{Photospheric Absorption Lines}

The broad, pronounced stellar wind features, fully discussed in the previous section, arise as intense radiation fields accelerate gas off of stellar surfaces. However, gas within the stellar photospheres also absorbs stellar radiation. These photospheric absorption lines are narrower and weaker than the stellar wind lines because the photosphere is relatively static, but they provide similarly robust indicators of stellar age and $Z_{*}$ (de Mello et al. 2000). Since photospheric gas is denser than interstellar gas, photospheric lines typically arise from excited states that are easily separated from ISM lines.

High-ionization photospheric lines, like S V $1502 \AA$, are found in the spectra of young stars, but the rest-frame FUV has a host of O-star diagnostics, such as stellar wind lines, that are stronger features and diagnose the stellar population properties at a lower $\mathrm{S} / \mathrm{N}$. The photospheric lines excel in diagnosing older ( $>7 \mathrm{Myr}$ ), B-star-dominated populations (de Mello et al. 2000), because the photospheric features are the only distinguishing features of B-stars in the FUV continuum. Previous authors have suggested that individual lines (see Table 1; de Mello et al. 2000) and bands of photospheric lines near 1417 and 1935-2020 ̊ identify B-star populations (Rix et al. 2004).

Our moderate-resolution data reveal that the C III and Si III photospheric lines at $1247 \AA$ (see Figure 6) and between 1295 and $1299 \AA$ (specifically Si III $1299 \AA$; see Figure 9) are the strongest due to their low excitation energies $(6 \mathrm{eV}$ versus $10-30 \mathrm{eV}$ for the features near $1420 \AA$ A Leitherer et al. 2011). The $1290 \AA$ lines blend with neighboring O I and Si II ISM

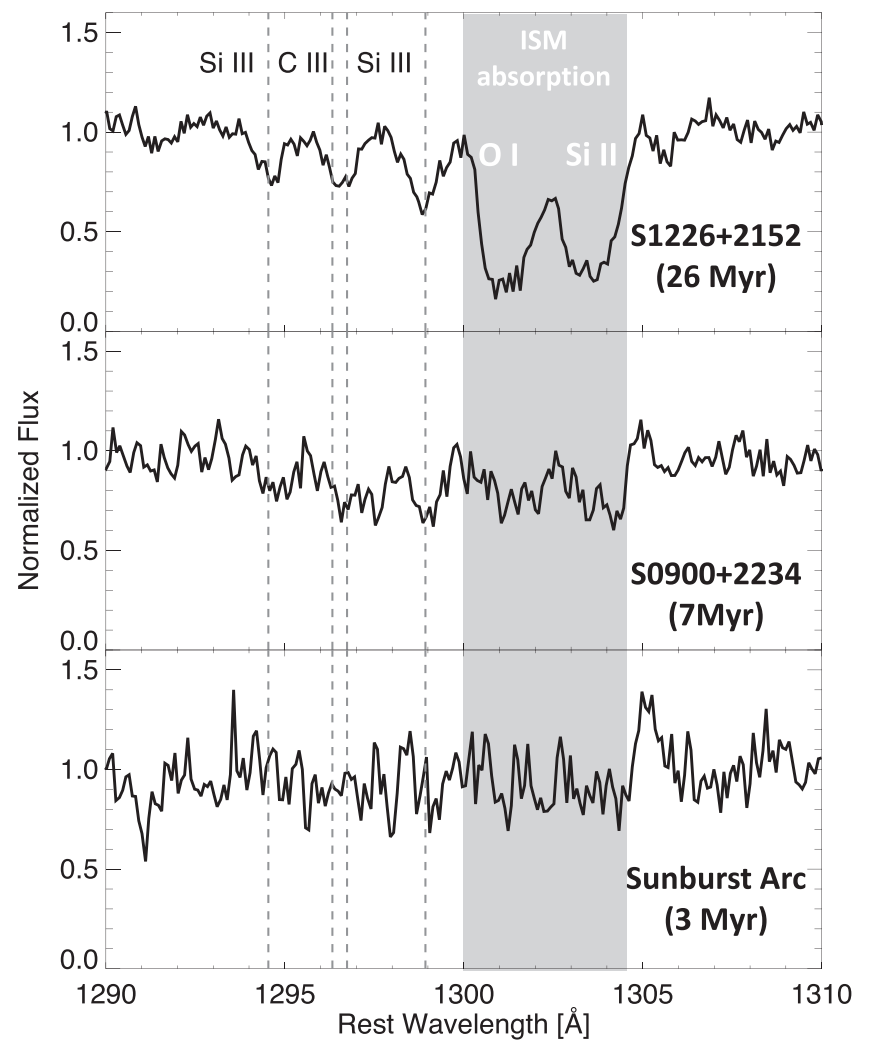

Figure 9. Set of photospheric absorption lines (1290-1300 А) from three galaxies: S1226+2152 (top panel), S0900+2234 (middle panel), and the Sunburst Arc (bottom panel). The four photospheric absorption lines are marked by dashed gray lines. These lines originate in the photospheres of B-type stars with ages greater than $8 \mathrm{Myr}$ and strengthen with increasing lightweighted age (printed underneath each name). Gray regions indicate possible ISM absorption from O I $1302 \AA$ and Si II $1304 \AA$.

absorption features at low spectral resolution, but at moderate resolution the Si III $1299 \AA$ line is resolved. Figure 3 clearly shows that $\mathrm{Si}$ III strengthens in stellar populations older than 5-10 Myr. The Si III $1299 \AA$ equivalent width decreases from $0.40 \pm 0.02 \AA$ for $\mathrm{S} 1226+2152$ with an age of $26 \mathrm{Myr}$, to $0.29 \pm 0.15$ for $\mathrm{S} 0900+2234$ with an age of $7 \mathrm{Myr}$, to undetected $(0.07 \pm 0.05 \AA)$ for the Sunburst Arc at 3 Myr. Similarly, the other three MEGaSaURA galaxies with ages less than 6 Myr (RCS Knot E with an age of $2 \mathrm{Myr}$, S0033+0242 with an age of $5 \mathrm{Myr}$, and RCS Knot $\mathrm{U}$ with an age of $5 \mathrm{Myr}$ ) have undetected Si III $1299 \AA$ (equivalent widths of $0.12 \pm 0.21,0.05 \pm 0.07$, and $0.08 \pm 0.17 \AA$, respectively). Very young populations do not contain the C III 1299 A photospheric feature.

There are multiple Fe photospheric lines in the FUV that arise from the Fe III, Fe IV, and Fe V ionization states (Table 1; Nemry et al. 1991; de Mello et al. 2000; Rix et al. 2004). These $\mathrm{Fe}$ lines (specifically $\mathrm{Fe} \mathrm{V}$ in O-stars) blanket the spectral regions, overlap in wavelength, and form large-scale continuum features that change the shape of the stellar continuum. These Fe features are included in the full spectral fitting, but even at the high $\mathrm{S} / \mathrm{Ns}$ of the MEGaSaURA sample, the Fe lines are very weak.

The power and utility of the stellar photospheric lines are limited because the photospheric lines are significantly weaker than the stellar wind features, requiring extremely high $\mathrm{S} / \mathrm{N}>20$ observations. At the $\mathrm{S} / \mathrm{N}$ and spectral resolution of our observations, the most prominent photospheric lines are 


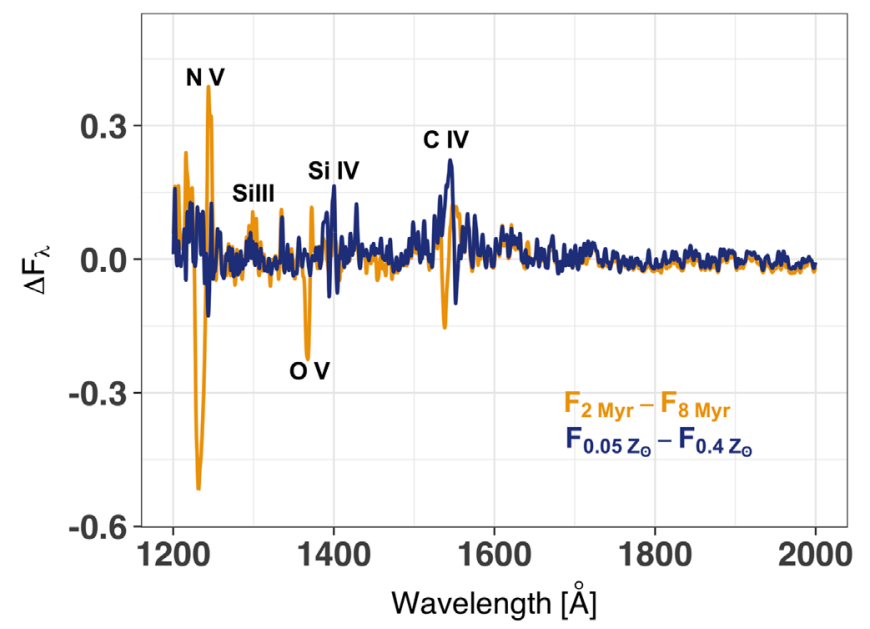

Figure 10. Change in flux $\left(\Delta F_{\lambda}\right)$ incurred by increasing the age from 2 to $8 \mathrm{Myr}$ of a $0.2 Z_{\odot}$ population (gold line) or by increasing the stellar metallicity from $0.05 Z_{\odot}$ to $0.4 Z_{\odot}$ of a $5 \mathrm{Myr}$ population (blue line). The strong spectral features discussed in the text are labeled, but significant continua changes occur outside of these spectral features in "featureless" regions (see Table 2).

near $1299 \AA$, and these features indicate the presence of stars with ages $>7$ Myr.

\subsection{Summary: Individual Spectral Features Are Consistent with Inferred Stellar Ages and Metallicities}

Throughout this section, we have compared the lightweighted stellar population properties (age and metallicity) to individual stellar features, but in all cases the inferred stellar properties were determined from the entire observed wavelength regime. In Figure 10 we plotted the change in flux density $\left(\Delta F_{\lambda}\right)$ that occurs while varying the stellar age (gold) or metallicity (blue). The largest $\Delta F_{\lambda}$ arises from the strong stellar wind and photospheric lines that we emphasized above. We demonstrated this by integrating the $\sqrt{\Delta F_{\lambda}^{2}}$ within specific wavelength regions to determine the total flux change attributable to individual spectral regions (Table 2). The $\mathrm{N} \mathrm{V}$ profile changes almost exclusively with age and is weakly dependent on $Z_{*}$. Similarly, the Si IV region largely depends on $Z_{*}$ and hardly depends on stellar age (compare Columns 3 and 4 in Table 2). While the notable stellar wind features dominate Figure 10, small spectral features occur outside these regions that in aggregate differentiate between stellar properties. For instance, the region between 1420 and $1520 \mathrm{~A}$ is devoid of prominent stellar wind features, but the shape of the stellar continuum noticeably changes over $100 \AA$ due to the stellar population age and $Z_{*}$. The $1420-1520 \AA$ region changes the flux comparably to the broad C IV P-Cygni wind feature and the Si III $1299 \AA$ photospheric regions, and has more power to differentiate stellar properties than the Si IV wind region. This implies that "featureless" regions of stellar continuum hold significant power to determine stellar age and $Z_{*}$. The power in these regions stems from the fact that they are not featureless, but crucially contain weak photospheric metal features like Fe III, Fe IV, and Fe V (Nemry et al. 1991; de Mello et al. 2000; Rix et al. 2004). In other words, the previous sections were not advocating the use of a single feature to determine the stellar properties; rather, the sections illustrate that the spectral features consistently and distinctly vary according to the inferred stellar population properties from the full stellar continuum.
Table 2

Integrated $\sqrt{\Delta F_{\lambda}^{2}}$ Incurred When Changing Stellar Population Properties

\begin{tabular}{lccc}
\hline \hline Region & Spectral Feature within Region & $\Delta$ Age & $\Delta Z_{\odot}$ \\
\hline $1225-1250$ & N V & 7.3 & 1.4 \\
$1250-1350$ & Si III 1299 & 2.4 & 2.1 \\
$1380-1410$ & Si IV & 0.8 & 1.7 \\
$1420-1520$ & Featureless & 2.0 & 2.4 \\
$1530-1560$ & C IV & 2.6 & 3.1 \\
$1660-1760$ & Featureless & 1.3 & 1.7 \\
$1900-2000$ & Featureless & 1.6 & 1.0 \\
\hline
\end{tabular}

Note. Integrated root squared flux difference in a given wavelength region (Column 1) that includes the spectral features discussed in the text (Column 2). Column 3 gives the root square flux difference of increasing the age of a $0.2 Z_{\odot}$ STARBURST99 model from 2 to $8 \mathrm{Myr}$ (gold line in Figure 10) integrated over the region in Column 1. Column 4 gives the same value but for increasing the stellar metallicity from $0.05 Z_{\odot}$ to $0.4 Z_{\odot}$ for a $5 \mathrm{Myr}$ population (blue line in Figure 10).

Young stellar populations produce the most ionizing photons. Thus, it is important to have spectral features that distinguish immense sources of ionizing photons. There are spectral indices that are unique to very young $(<7 \mathrm{Myr})$ stellar populations: (1) strong and broad C IV and N V P-Cygni features (Figure 3), (2) broad $\left(>300 \mathrm{~km} \mathrm{~s}^{-1}\right.$ ) He II emission (Figure 8), and (3) undetected Si III $1299 \AA$ photospheric absorption features (equivalent widths $<0.1 \AA$; Figure 9). These spectral signatures suggest that the stellar population is dominated by stars younger than $7 \mathrm{Myr}$. Older stellar populations appear to have combinations of both young and old stars, obscuring single trends in spectral features (see Section 5.3).

\section{Discussion}

\subsection{Nebular Gas and Massive Stars Have Similar Metallicities}

Stellar metallicity is a fundamental galaxy property that is traditionally challenging to measure but crucially determines the production of ionizing photons. Previous observations have either used spectral indices (e.g., Rix et al. 2004; Leitherer et al. 2011; Byler et al. 2018) or full spectral synthesis (e.g., Pettini et al. 2000; Kudritzki et al. 2012; Steidel et al. 2016, 2018; Hernandez et al. 2019) to estimate the metallicities of young stellar populations. Here, we explore the relation between the light-weighted $Z_{*}$ derived from the full spectral synthesis to the observed gas-phase metallicities derived from rest-frame optical nebular emission lines $\left(Z_{\text {neb }}\right)$.

Figure 11 shows that the light-weighted $Z_{*}$ and the $Z_{\text {neb }}$ are correlated at the $8 \sigma$ significance (with a Pearson's correlation coefficient of 0.87 ). The metallicities scatter about the one-toone line, indicating that the young, massive stars have a metallicity similar to the surrounding nebular gas. Both the lowand high-redshift galaxies are near the one-to-one line; this relationship does not appear to evolve with redshift. There is a dearth of points above $1 Z_{\odot}$, largely as a selection effect of both samples. While we have used direct metallicities whenever possible (see the discussion in Section 2.3), we have used different metallicity calibrations, which can have a 0.4 dex scatter in their relative metallicity calibrations. The largest outlier from a one-to-one relationship is NGC 3256, in the lower right quadrant with $Z_{*}=0.59 \pm 0.13 Z_{\odot}$ and $Z_{\text {neb }}=1.10 \pm 0.25 Z_{\odot}$ (including calibration uncertainties; Engelbracht et al. 2008). 


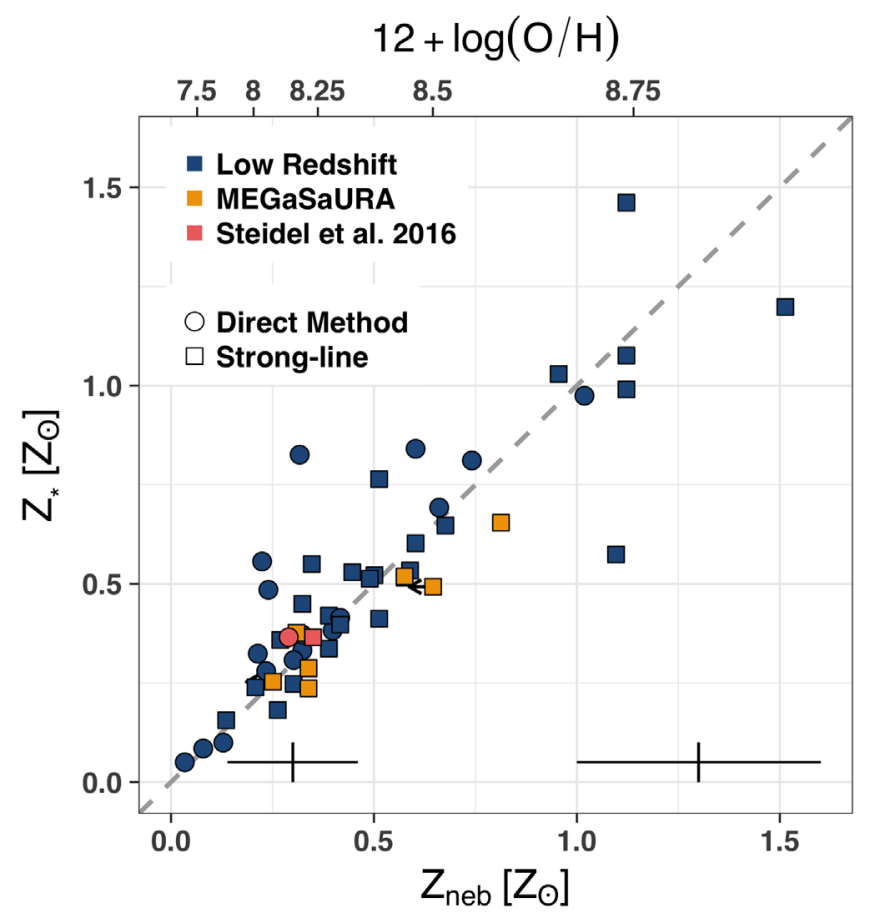

Figure 11. Comparison of the light-weighted stellar metallicity $\left(Z_{*}\right)$ derived from the STARBURST99 fitting to the gas-phase nebular metallicity $\left(Z_{\mathrm{neb}}\right)$. The points are color coded according to the three samples: blue are low-redshift galaxies, orange are the individual $z \sim 2$ galaxies from the MEGaSaURA sample, and the red points arethe composite spectrum from Steidel et al. (2016). We include both the direct and strong-line (O3N2) metallicity calculations from Steidel et al. (2016). The symbol shapes correspond to whether the $Z_{\text {neb }}$ was calculated using the direct method (circles) or a strongline calibration (squares). Note that only eight of the 19 MEGaSaURA galaxies have a measured $Z_{\text {neb. }}$ Two error bars, one at $0.3 Z_{\odot}$ and one at $1.2 Z_{\odot}$, are shown in the bottom of the plot using the median $Z_{*}$ error and the 0.4 dex 12 $+\log (\mathrm{O} / \mathrm{H})$ spread between $Z_{\text {neb }}$ diagnostics suggested by Kewley \& Ellison (2008). A one-to-one line is shown in gray: $Z_{*}$ is largely equivalent to $Z_{\text {neb. }}$

Consequently, the $Z_{\text {neb }}$ of the largest outlier is still consistent with $Z_{*}$ at the $1.3 \sigma$ significance level.

Further, we measure the residual standard error of the trend to be $0.16 Z_{\odot}$, or $0.17 \mathrm{dex}$ in $12+\log (\mathrm{O} / \mathrm{H})$ at the median $Z_{\text {neb }}$ of $0.4 Z_{\odot}$. This residual standard error is consistent with the full 0.4 dex spread found by Kewley \& Ellison (2008) between the Pettini \& Pagel (2004) and Kobulnicky \& Kewley (2004) metallicity calibrations (the two most commonly used strongline methods here). Thus, the observed dispersion in Figure 11 is entirely consistent with the spread of the different $12+\log$ $(\mathrm{O} / \mathrm{H})$ calibration methods.

The $Z_{*}$ is surprisingly consistent for the lowest metallicity populations. The stellar mass-loss rate, and in turn the stellar wind profile, is only empirically constrained for $Z_{*}>0.2 Z_{\odot}$, and the $0.05 Z_{\odot}$ STARBURST99 models instead rely on an extrapolation of the mass-loss rates to lower metallicities (Leitherer et al. 1992; Vink et al. 2001). The close metallicity correspondence below $0.2 Z_{\odot}$ suggests that the stellar wind extrapolation adequately reproduces the observed stellar wind profiles and their mass-loss rates. Note that $Z_{\text {neb }}$ for $1 \mathrm{Zw} 18$ is lower than $0.05 Z_{\odot}$, the lowest Meynet et al. (1994) atmospheric model, leading to a possible overestimate of the lightweighted $Z_{*}$ for this galaxy.

The strong relationship between $Z_{\text {neb }}$ and $Z_{*}$ in Figure 11 suggests that $Z_{*}$ robustly determines the metallicities of galaxies. A robust metallicity indicator is needed at high redshifts because the crucial, yet extremely faint, metallicitysensitive lines, like [O III] $4363 \AA$, are redshifted out of the optical (Yuan \& Kewley 2009; James et al. 2014b; Sanders et al. 2016). Recent work has attempted to calibrate $Z_{\text {neb }}$ using rest-frame UV emission lines (Pérez-Montero \& Amorín 2017; Byler et al. 2018), but these calibrations depend on rather uncertain assumptions of the carbon-to-oxygen abundance that are not yet well calibrated. Fortunately, Figure 11 implies that observations from the ground using upcoming large telescopes and the rest-frame FUV stellar continuum may constrain the chemical enrichment of galaxies to redshifts of $z \sim 6$.

The similarity of $Z_{\text {neb }}$ and $Z_{*}$ indicates that the gas surrounding high-mass stars is not instantaneously metalenriched by massive stars. In other words, it takes longer than the inferred lifetimes of the massive star populations to increase the metallicity of adjacent ISM gas. Newly synthesized metals are ejected from star-forming galaxies as very hot supernovae ejecta, and galaxies must take longer than the $10^{7} \mathrm{yr}$ timescales observed here to fully mix these newly synthesized metals (Kobulnicky \& Skillman 1997). In fact, we did not find a statistical correlation between $Z_{\text {neb }}$ and the stellar age, implying either that gaseous enrichment occurs on longer timescales than observed here, or that the enrichment of a single stellar population is within the scatter of Figure 11.

\subsection{Ionizing Continua of Massive Stars}

The non-ionizing FUV continua of massive stars are rich with features that reveal the stellar population age and metallicity. The primary goal of this paper is to use these inferred light-weighted metallicities and ages to constrain the ionizing continua of massive stars. The same massive stars that produce the FUV spectral features also produce the unseen ionizing continua; these spectral features are the most direct probe of the ionizing continua because they only depend on the massive star properties (unlike nebular emission lines, which also depend on the gaseous properties). We therefore use the theoretical stellar models to develop simple prescriptions for how the ionizing continuum varies with stellar properties (Section 5.2.1) and then infer the ionizing continua from the FUV observations using the stellar fits (Section 5.2.2). We refer to the ionizing continua determined from the models as the inferred ionizing continua to emphasize that we do not directly observe the ionizing continua. Throughout this subsection we only focus on the STARBURST99 fits; we return to the BPASS fits in Section 5.4.

\subsubsection{Stellar Population Properties Determine the Ionizing Continua of Massive Stars}

The full ionizing continuum will likely never be observed because foreground neutral hydrogen efficiently absorbs these photons. Observations are typically fortunate to observe the ionizing continuum at a single wavelength, which is most feasible at $900 \AA$. This ionizing flux density is then normalized by the observed non-ionizing continuum to control for stellar mass and star formation contributions. Thus, the literature typically quantifies the ionizing continuum through the ratio of the flux at $900 \AA$ (ionizing) to the flux at $1500 \AA$ (nonionizing), or $F_{900} / F_{1500}$ (Steidel et al. 2001). In this subsection, we use the STARBURST99 fully theoretical models to explore 


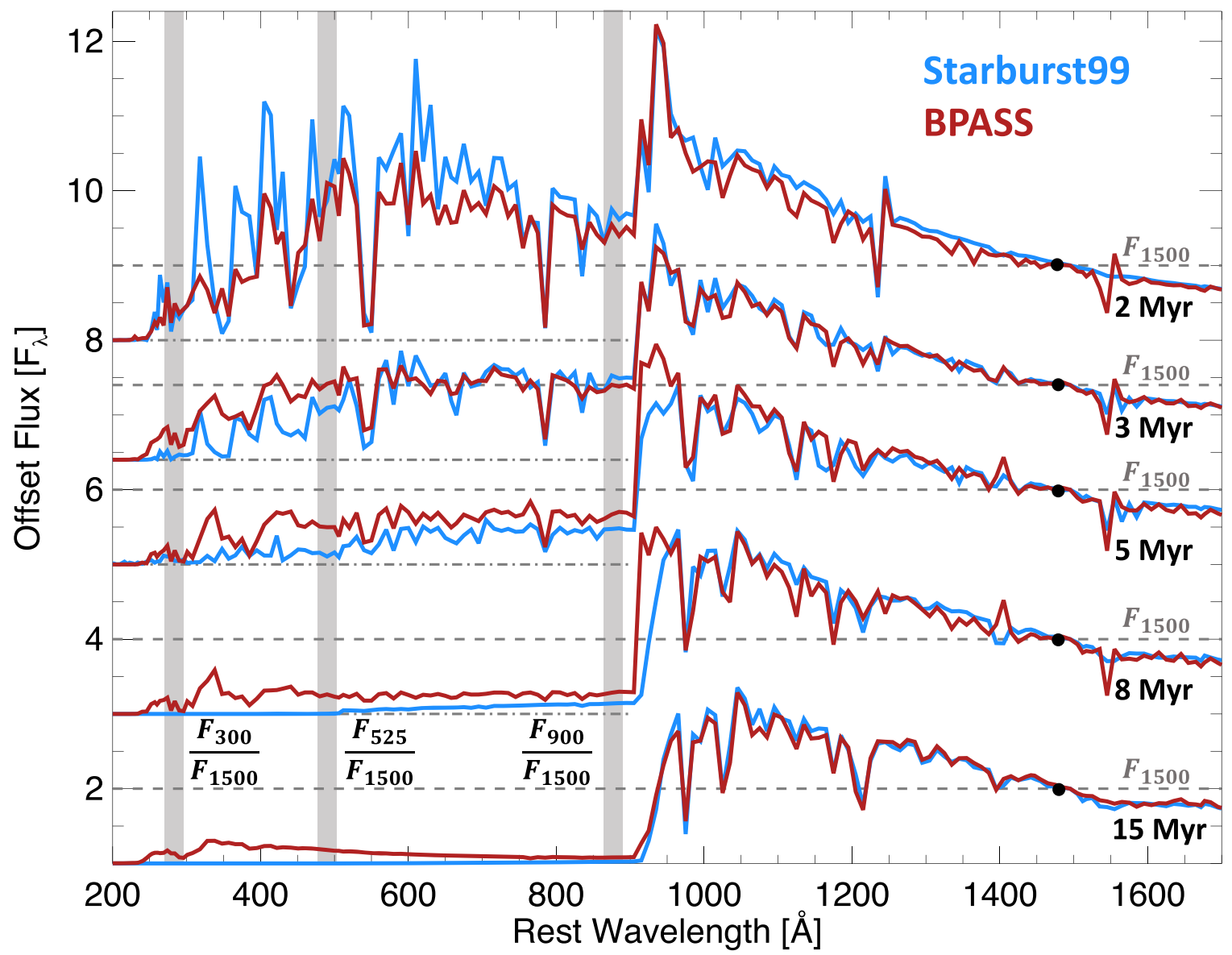

Figure 12. Ionizing continua of five different single-age, fully theoretical stellar models with a constant metallicity of $0.4 Z_{\odot}$. We plot both STARBURST 99 single-star models (blue lines) and BPASS models (red), which include binary star evolution. The stellar populations are ordered by descending age, with the youngest (2 Myr) population at the top and the oldest (15 Myr) population at the bottom. Each age is offset by a constant for presentation purposes. Each population is normalized by the flux density at $1500 \AA$ ( $F_{1500}$; dashed line) such that zero flux is given by the dotted-dashed line. The gray regions highlight the three regions where we measure the ratio of the ionizing to non-ionizing flux density to quantify the shape of the ionizing continuum: $F_{300} / F_{1500}, F_{525} / F_{1500}$, and $F_{900} / F_{1500}$ (the flux density at 300,525 , and $900 \AA$ relative to the flux density at $1500 \AA$ ). Here, $F_{900} / F_{1500}$ steadily decreases as the stellar population ages.
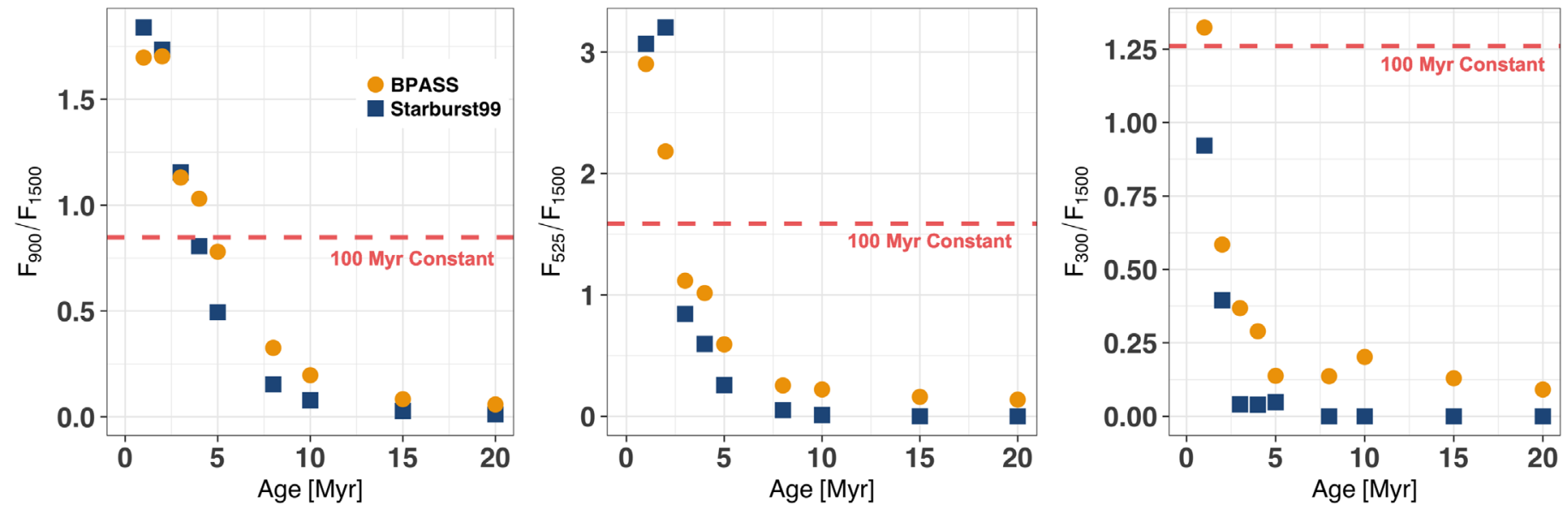

Figure 13. Temporal variation of the ratio of the ionizing to non-ionizing flux density at three ionizing wavelengths (900, 525, and $300 \AA$ from left to right) of a theoretical, $0.4 Z_{\odot}$, single-burst stellar population. We include both STARBURST99 (blue squares) and BPASS models (gold circles). Young stellar populations emit more ionizing photons than non-ionizing photons. The flux ratio of a BPASS model with a constant star formation law and a $0.05 Z_{\odot}$ metallicity is included as a dashed red line.

how the stellar properties determine this ratio as well as flux density ratios at other ionizing to non-ionizing wavelengths.

Figure 12 shows the theoretical ionizing continua of singleburst, $0.4 Z_{\odot}$ STARBURST99 stellar populations at five different ages. At 2 Myr the intrinsic ionizing flux density at $900 \AA$ is actually 1.7 times more luminous than the non-ionizing flux density at $1500 \AA$. This is because the blackbody spectrum of a $43,000 \mathrm{~K}$ object, or a $40 M_{\odot}$ star, peaks at $670 \AA$. The ratio 

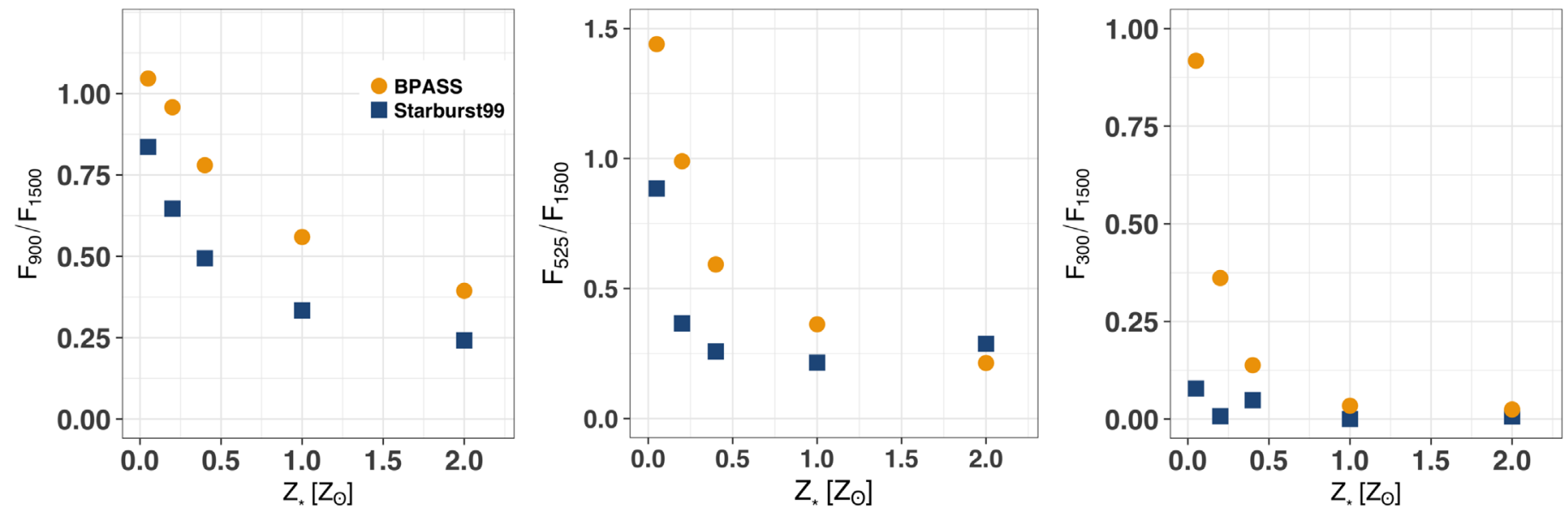

Figure 14. Variation of the ionizing to non-ionizing flux density ratios with stellar metallicity $\left(Z_{*}\right)$. The three panels show these ratios at three wavelengths (900, 525 , and $300 \AA$ from left to right) for a theoretical 5 Myr single-burst stellar population. We include both STARBURST99 (blue squares) and BPASS models (gold circles). Low-metallicity BPASS models produce significantly more ionizing photons, especially at $300 \AA$, due to the effects of binary star evolution (Section 5.4.1).

$F_{900} / F_{1500}$ steadily decreases as the stellar population ages, from 1 at 3 Myr to 0 at 15 Myr. Clearly, the stellar age crucially determines the stellar ionizing flux density of single bursts.

We use three different ionizing to non-ionizing flux density ratios to quantify the shape of the ionizing continuum $\left(F_{900} / F_{1500}, F_{525} / F_{1500}, F_{300} / F_{1500}\right)$. As hinted in Figure 12 , the left panel of Figure 13 shows a smooth temporal trend of $F_{900} / F_{1500}$ with age at a fixed metallicity. Higher energy photons (probed by the flux density ratio of $F_{525} / F_{1500}$ ) have a similarly strong temporal evolution (middle panel of Figure 13), but shifted to earlier ages, such that only stars younger than $5 \mathrm{Myr}$ are sufficiently hot to emit appreciably at $525 \mathrm{~A}$. The ionizing continua of the youngest stars peak near $525 \AA$, where $F_{525}$ is three times more luminous than $F_{1500}$. Finally, the highest energy photons (probed by the flux density ratio $F_{300} / F_{1500}$ ) are exclusively produced by stellar populations with ages less than $2 \mathrm{Myr}$ (right panel of Figure 4). The flux density ratios of theoretical STARBURST99 models show that the stellar age correlates with the shape and strength of the ionizing continua.

The stellar metallicity $\left(Z_{*}\right)$ also affects the shape of the ionizing continuum as measured by the flux density ratios (Figure 14; Smith et al. 2002). At a constant stellar age of $5 \mathrm{Myr}$, the $F_{900} / F_{1500}$ increases by a factor of 3 as $Z_{*}$ decreases by a factor of 40 (left panel of Figure 14). Similarly, $F_{525} / F_{1500}$ and $F_{300} / F_{1500}$ decrease by a factor of 3 and 12 from $Z_{*}=0.05$ to $2 Z_{\odot}$, respectively. The ionizing to non-ionizing flux density ratios also quantify the impact of $Z_{*}$ on the shape and strength of the stellar ionizing continua.

Figure 15 illustrates that both the stellar population age and metallicity affect the ionizing continuum. A multivariate, robust M-estimator linear regression (Huber 1981) finds a relationship between these three variables for the single-burst STARBURST99 models (only including ages $<20 \mathrm{Myr}$ ) as

$$
\frac{F_{900}}{F_{1500}}=(3.5 \pm 0.3) \times 10^{-(0.14 \pm 0.01)\left(\frac{\mathrm{Age}}{1 \mathrm{Myr}}\right)-(0.21 \pm 0.02)\left(\frac{Z_{*}}{1 Z_{\odot}}\right) .}
$$

This relation determines the ratio of the intrinsic ionizing flux density at $900 \AA$ to the non-ionizing flux density at $1500 \AA$ for a single-age burst of star formation given the stellar population age and metallicity.

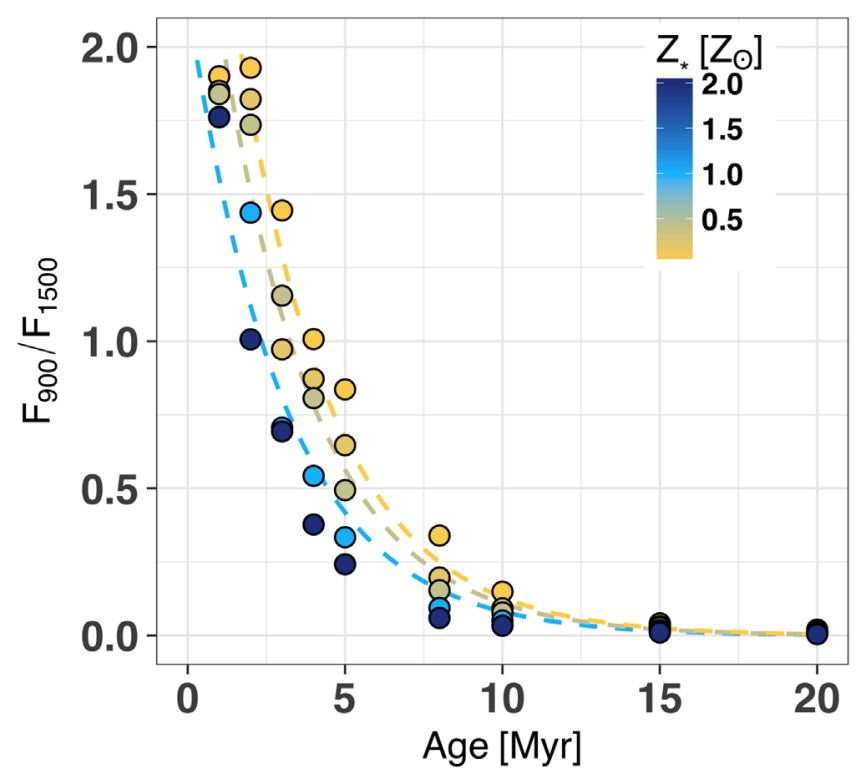

Figure 15. Theoretical evolution of the ionizing to non-ionizing flux density ratio $\left(F_{900} / F_{1500}\right)$ with stellar age of a single-burst STARBURST99 model. All five STARBURST99 metallicities are included (as indicated by the color bar). After $10 \mathrm{Myr}$, a single burst produces an insignificant amount of ionizing photons. The colored curves are single-metallicity trends, using Equation (5), for three metallicities color coded the same as the points (light blue, tan, and gold are $1,0.4$, and $0.05 Z_{\odot}$, respectively).

In Figure 15, we use Equation (5) to overplot three curves of constant $Z_{*}$ onto the full STARBURST99 grid. The curves generally approximate the variations in the models, but real variations still exist at a given age. Here, $F_{900} / F_{1500}$ evolves significantly at the youngest ages, and small measurement errors lead to large $F_{900} / F_{1500}$ errors. The median error of the estimated age and $Z_{*}$ is $13 \%$ and $11 \%$ of the estimated values, respectively. For an estimated age of $3 \mathrm{Myr}$ and metallicity of $0.4 Z_{\odot}$, with these median uncertainties, there is a $15 \%$ uncertainty on $F_{900} / F_{1500}$, including calibration errors. The median MEGaSaURA S/N, 21, is high for rest-frame FUV observations, but extremely high-quality observations are required to determine $F_{900} / F_{1500}$, even with a $15 \%$ error.

The $F_{900} / F_{1500}$ scales strongly with both stellar age and metallicity because both affect the stellar temperature. As stellar populations age, their stellar temperatures decrease, 


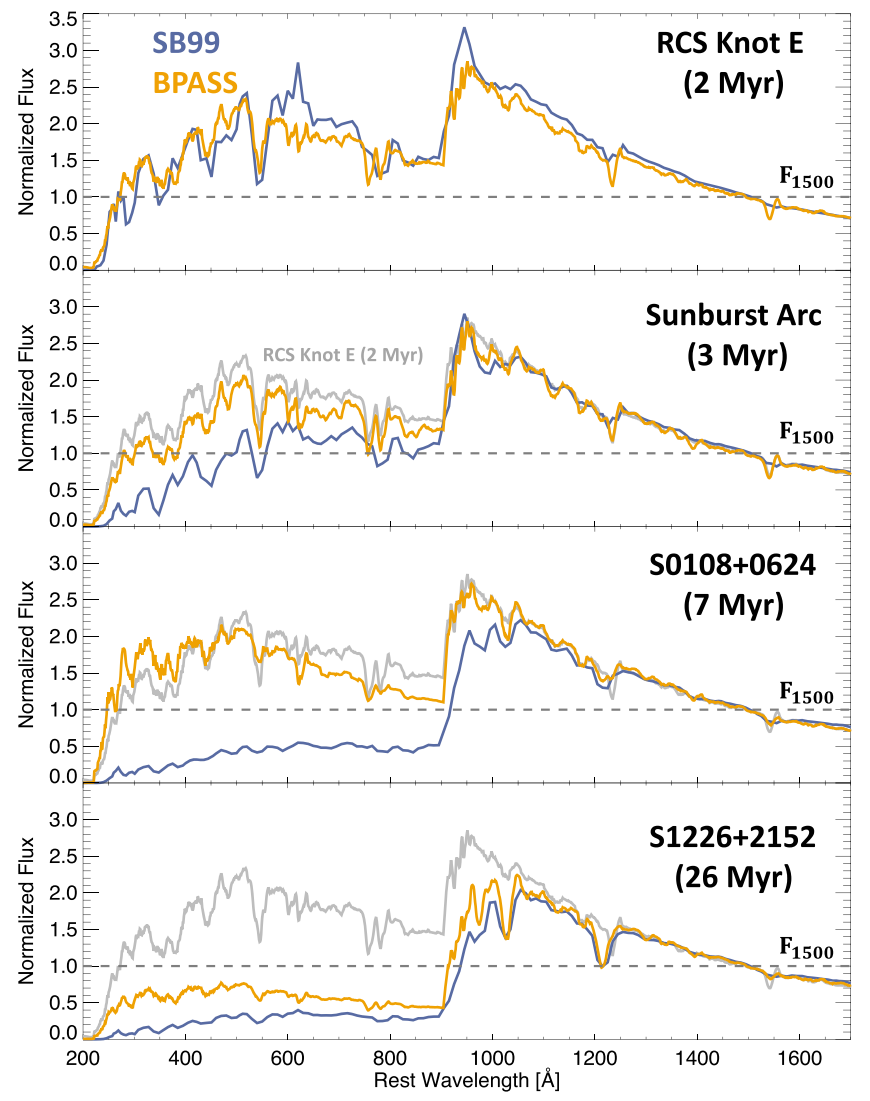

Figure 16. Inferred ionizing continua, normalized at $1500 \AA$, for four galaxies: RCS Knot E (with a STARBURST99 light-weighted age of $2 \mathrm{Myr}$; top panel), the Sunburst Arc (3 Myr; second panel), S0108+0624 (7 Myr; third panel), and S1226+2152 (26 Myr; bottom panel). Both the STARBURST99 (blue line) and BPASS models (gold line) are plotted to illustrate the large differences between the inferred populations at older light-weighted ages. The spectra are ordered by increasing light-weighted age. The RCS Knot E inferred BPASS continuum is included in gray in all lower panels to illustrate how the ionizing continuum changes as the stellar population ages.

which increases the fraction of neutral hydrogen within the stellar atmospheres. This is observed in the optical, where Balmer absorption lines increase in older B- and A-stars. Thus, the rapidly evolving $F_{900} / F_{1500}$ ratio with stellar age and $Z_{*}$ in Figure 15 probes the increasing $\mathrm{X}\left(\mathrm{H}^{0}\right) / \mathrm{X}\left(\mathrm{H}^{+}\right)$ionization fraction within the stellar atmospheres with decreasing stellar temperature. By the time the stellar population ages to $15 \mathrm{Myr}$, the temperature has dropped below $20,000 \mathrm{~K}$, and there is sufficient neutral hydrogen within the stellar atmospheres to absorb all of the ionizing photons. Thus, $F_{900} / F_{1500}$ traces the hydrogen ionization fraction within the stellar atmospheres.

\subsubsection{Inferred Ionizing Continua from Observed Galaxies}

We inferred the ionizing continua of the observed massive star populations by extending the stellar fits blueward beyond the observations. The fits are linear combinations of multiple single-age models, and therefore the inferred ionizing continua may be more complicated than the single-burst relations presented in Section 5.2.1. How much do the inferred continua depart from a single-burst model?

In Figure 16, we show the inferred ionizing continua of four galaxies that span the full age range of the sample. Focusing on only the STARBURST99 fits (blue lines), the general trend found in Figure 12 is reproduced: the inferred ionizing continua at
$900 \AA$ decrease with fitted stellar age from RCS Knot E (top panel; $2 \mathrm{Myr}$ ), to the Sunburst Arc (second panel; $3 \mathrm{Myr}$ ), to S0108+0624 (third panel; 7 Myr), and finally S1226+2152 (bottom panel; 26 Myr). The galaxies with the strongest inferred ionizing continua are the youngest galaxies with the strongest stellar wind profiles (Section 4).

At wavelengths blueward of $900 \AA$, the STARBURST 99 inferred ionizing continua have substantially different morphologies. The ionizing continuum of RCS Knot E increases from 900 to $525 \AA$, the Sunburst Arc stays relatively flat from 900 to $525 \AA$, while the ionizing continua of S0108+0624 and S1226 +2152 both decline with decreasing wavelength. The inferred shapes of the ionizing continua constrain the total number of ionizing photons produced by the stellar population and the hardness of the nebular emission spectra (see Section 5.7). In this parlance, RCS Knot E has a particularly hard ionizing spectrum, while $\mathrm{S} 1226+2152$ has a relatively soft spectrum.

The inferred $F_{900} / F_{1500}$ qualitatively follows the general trends outlined in Section 5.2.1. RCS Knot E is the youngest population and has a $F_{900} / F_{1500}$ that is five times larger than the oldest galaxy, S1226+2152. However, there are large departures in the inferred $F_{900} / F_{1500}$ values from the singleburst models: S1226+2152 has a light-weighted age of $26 \mathrm{Myr}$, yet it still has a $F_{900} / F_{1500}=0.3$. S1226+2152 has an old stellar population, the C IV profile is nearly flat (Figure 4), yet the inferred ionizing continuum is 500 times stronger than a single-burst model with the same age and metallicity $\left(F_{900} / F_{1500}=0.0006\right)$. Similarly, S1527+0652 and the Cosmic Eye have light-weighted ages of 21 and $29 \mathrm{Myr}$ but inferred $F_{900} / F_{1500}$ of 0.2 and 0.1 , respectively. These values are 50 and 400 times larger than predicted by the single-burst models. This overproduction of ionizing photons extends to all populations older than $9 \mathrm{Myr}$.

Whether the inferred ionizing continua agree with the singleburst models splits the observed stellar populations into two star formation histories: a single-burst and a mixed-age population (Figure 17). Bursts have stellar ages less than 8.6 Myr, while mixed-age populations have ages greater than 8.6 Myr. This divides the full sample into 31 burst-dominated stellar populations and 30 mixed-age populations. There is not a statistical redshift dependence as both the low-redshift sample and the MEGaSaURA sample are nearly split evenly.

We overlay the single population model tracks of Figure 15 onto the inferred $F_{900} / F_{1500}$ in Figure 17 to show that young populations have single-burst-dominated ionizing spectra. All of the burst-dominated non-ionizing spectra have the spectral properties outlined in Section 4.3 for young stellar populations: strong $\mathrm{N} \mathrm{V}$ and C IV P-Cygni features, broad He II emission (when the transition is within the wavelength coverage), and nondetected Si III photospheric features.

The spectra of the second population are a mixture of old and young stellar populations. The stellar continua of these stellar populations are complex, with contributions from young stars shaping the stellar winds and B-stars contributing photospheric absorption features. This creates an averaged ionizing continuum that is nonzero due to contributions from massive O-stars, but diluted by contributions from older populations.

The spectral differences between the two populations are seen by comparing the C IV features of the burst-dominated RCS Knot E and the mixed-age S1226+2152 in Figure 4. RCS Knot E has very strong and pronounced C IV absorption and emission profiles, similar to the single-age STARBURST99 


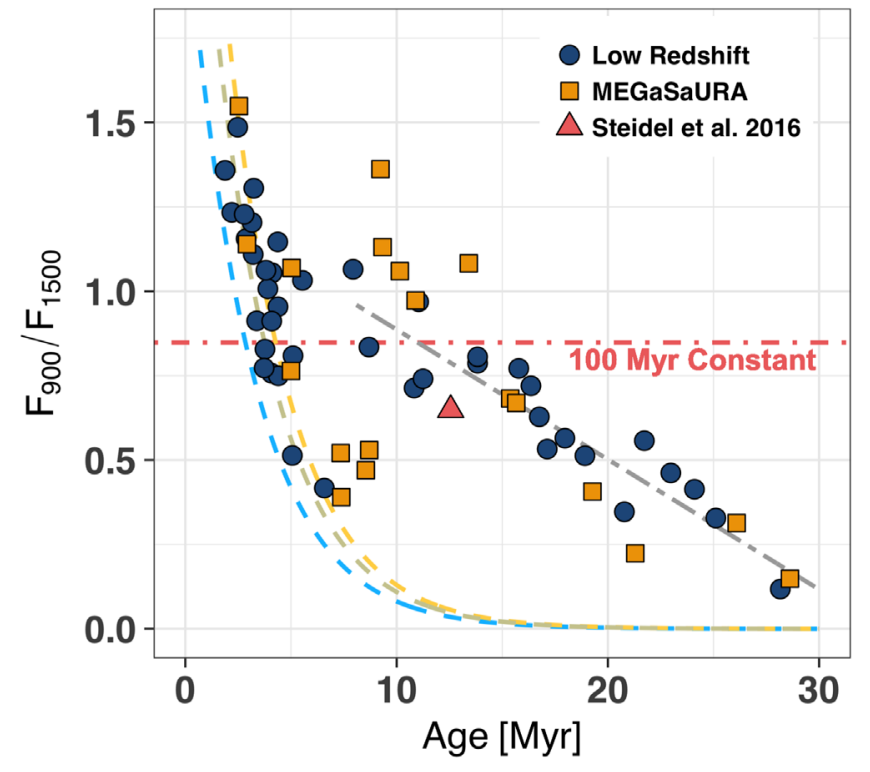

Figure 17. Inferred ratio of the ionizing to non-ionizing flux density $\left(F_{900} /\right.$ $\left.F_{1500}\right)$ vs. the inferred age from the multiple-age STARBURST99 stellar continuum fits to the MEGaSaURA (gold squares) and low-redshift (blue circles) samples. The curves are the single-burst STARBURST99 models at three metallicities from Figure 15 (blue is $1 Z_{\odot}$, tan is $0.4 Z_{\odot}$, and gold is $0.05 Z_{\odot}$ ). The observed points are described by two star formation histories: a single burst of star formation that aligns with the single-burst models at young ages, and a mixed-age population. The gray line is the fit to the mixed-age population (Equation (14)). The red dashed line is the $F_{900} / F_{1500}$ value of a $0.05 Z$ constant star formation BPASS model, and the red triangle is the value inferred from the STARBURST99 fit to the Steidel et al. (2016) spectra.

model. Meanwhile, single-burst models do not fit S1226 +2152: the $20 \mathrm{Myr}$ single-age C IV profile underestimates the C IV absorption and emission, indicating a weak O-star population. Besides this weak O-star contribution, the pronounced photospheric absorption clearly indicates an older B-star population (Figure 9). The observed spectral features demonstrate that as the inferred light-weighted age increases, a single stellar population ceases to dominate the stellar continuum; rather, the stellar light becomes a mix of young and old spectral features.

In Figure 17 , the $F_{900} / F_{1500}$ of the mixed-age population strongly $(5.5 \sigma$, Pearson's correlation coefficient of -0.70) and linearly decreases with light-weighted age between 8 and 30 Myr as

$$
\frac{F_{900}}{F_{1500}}=(1.35 \pm 0.09)-(0.042 \pm 0.005) \times\left(\frac{\mathrm{Age}}{1 \mathrm{Myr}}\right) \text {. }
$$

This relationship is overplotted on the observations in Figure 17 as a gray dotted-dashed line. There is not a statistically significant trend between $F_{900} / F_{1500}$ and $Z_{*}$, such that the statistical significance of the trend decreases if we introduce a multivariate fit. Equation (6) analytically quantifies the evolution of the strength of the ionizing continua of older mixed-aged stellar populations.

We investigated the origin of mixed-age populations by envisioning that the observations of individual star-forming regions sample a random mixture of stellar populations at various ages. This can be analytically tested using a random assortment of theoretical stellar models. The model grid contains more young stars because their spectral features vary on shorter timescales, so, for modeling purposes, we only included STARBURST99 models with ages less than 4 Myr or greater than $10 \mathrm{Myr}$. We then simulated whether a burst occurred using a binomial distribution with a uniform probability of success for each model. We chose the probability for success to be $15 \%$ such that the expected value of the number of bursts matched the number of models typically included in the fits $(\sim 6)$. The success probability was tested for a range between $1 \%$ and $50 \%$, but the results were not sensitive to the chosen probability. If the random binomial for a given model returned a success $\left(P\left(M_{i}\right)=1\right)$, we assigned a random light fraction drawn from a Gaussian distribution. This process was repeated for all possible stellar models, and the sum of all light fractions was normalized to total one. We multiplied the stellar models by the randomized light fractions and summed the synthetic stellar populations according to Equation (1). We inferred the light-weighted age and $F_{900} / F_{1500}$ using the same method as for the observations. We then repeated this process one million times to create a statistical sample of random mixtures of stellar populations. This sample of synthetic observations is hereafter referred to as the MCMC sample. We found a strong correlation (Pearson's correlation coefficient of -0.81 ) between the age and the $F_{900} / F_{1500}$ of this random mixed-age population, as

$$
\frac{F_{900}}{F_{1500}}=1.36-0.046 \times\left(\frac{\mathrm{Age}}{1 \mathrm{Myr}}\right) .
$$

This relationship is statistically similar to the relationship inferred from the mixed-age population in Equation (6). This suggests that older inferred light-weighted ages are composites of multiple epochs of stellar populations within a single aperture. A single stellar population does not dominate the FUV light of mixed-age populations; rather, the FUV light is broadly distributed over many ages. The resultant composite is a detailed, light-weighted mixture of the different epochs of star formation.

The relative proportions of this age mixture are determined by the light fraction of each model at a given age, or $f_{\text {age }}$ (where $f_{\text {age }}$ is the light fraction of each model age; see Equation (1)). As a simplified example, assume that the light at $1270 \AA$ of a $0.4 Z_{\odot}$ stellar population comes $70 \%$ from a $2 \mathrm{Myr}$ population $\left(f_{2}=0.7\right)$ and $30 \%$ from a $25 \mathrm{Myr}$ population $\left(f_{25}=0.3\right)$. The light-weighted age from Equation (3) is 8.9 Myr, and the lightweighted $F_{900} / F_{1500}$ from Equation (5) is 1.05 . These inferred values are on the age $-F_{900} / F_{1500}$ mixed-age relationship of Equation (6). The 8.9 Myr stellar continuum has a deep $\mathrm{N} \mathrm{V}$ profile from the $2 \mathrm{Myr}$ population, but weak photospheric lines from the $25 \mathrm{Myr}$ population. This hypothetical stellar population resembles RCS Knot $\mathrm{G}$ in Figure 4, which is dominated by light fractions of $f_{1}, f_{3}$, and $f_{40}$ of $0.30,0.43$, and 0.20 , respectively. If the assumed light fractions of the toy model are reversed, the light-weighted age increases to $18 \mathrm{Myr}$ and $F_{900} / F_{1500}$ decreases to 0.45 . The younger population contributes less to the integrated stellar light, and the stellar wind lines of this older hypothetical population are now flatter, resembling S1429-1202 in Figure 6. The observed stellar populations are more complicated than these toy examples, but they illustrate the evolution of the ionizing continua of mixedage populations.

The ionizing continuum of a mixed-age population looks very different from a single burst. A single-burst $8 \mathrm{Myr}$, $0.4 Z_{\odot}$ STARBURST99 population has $F_{900} / F_{1500}=0.2$ and 
$F_{300} / F_{1500}=0.05$, but the mixed-age population envisioned above has $F_{900} / F_{1500}=1.1$ and $F_{300} / F_{1500}=0.3$. This "old" mixed-age stellar population produces six times more photons per $F_{1500}$ than a single burst of the same age. Moreover, mixed-age populations produce a large number of extremely hard ionizing photons, due to the outsized presence of very young stellar populations (in this example, $f_{2}=0.7$ ). Similarly, the $18 \mathrm{Myr}$ population theorized above has an $F_{300} / F_{1500}$ that is still three times larger than a single-burst $3 \mathrm{Myr}$ stellar population, among the youngest in our sample, even though the stellar age inferred from the non-ionizing continuum is five times older. Mixed-age stellar populations produce dramatically more ionizing photons than suggested by their light-weighted ages.

The fitted light fractions illustrate the difference between a population dominated by a single burst and by a mixture of bursts (see Figure 2). RCS Knot E has a burst-dominated stellar spectra with a light-weighted age of $2.5 \pm 0.1 \mathrm{Myr}$. A full $100 \%$ of the FUV light comes from populations with ages less than $4 \mathrm{Myr}$ (and $f_{2}=61 \%$; left panel of Figure 2). Alternatively, S1527+0652 has a mixed-age stellar spectrum with a light-weighted age of $21 \pm 3 \mathrm{Myr}$. Young stellar populations ( $<5 \mathrm{Myr}$ ) within $\mathrm{S} 1225+2152$ contribute $19 \%$ of the FUV light, while older stellar populations supply $81 \%$ of the FUV light (where the dominant ones are $f_{10}$ and $f_{40}$ with $31 \%$ and $44 \%$, respectively). The age distribution of burst populations is relatively simple and clustered, but the light from mixed-age populations is broadly distributed over many stellar ages.

A principal worry is whether the derived continuum fits, and the associated mixed-age populations, are robust. The errors on the stellar properties were derived by varying the observed flux of the stellar continuum by a random Gaussian with a width equal to the observed flux uncertainty and then refitting the stellar continuum. This process was repeated 100 times, and the standard deviation of the ensemble was taken as the stellar property uncertainty. We find that the $\mathrm{S} / \mathrm{N}$ of the parameter scales with the spectral $\mathrm{S} / \mathrm{N}$ at the $3 \sigma$ significance ( $p$ value of 0.0006 , Kendall's $\tau$ value of 0.65 ). As a general guide, we find that the median $\mathrm{S} / \mathrm{N}$ of the inferred stellar population age is 0.44 times the observed spectral $\mathrm{S} / \mathrm{N}$ per resolution element. Thus, a spectral $\mathrm{S} / \mathrm{N}$ per resolution element of 22 (7) is required to determine the stellar age at the $10 \sigma(3 \sigma)$ significance level. In Section 5.2 we also noted that a spectral $\mathrm{S} / \mathrm{N}$ of 21 estimates the $F_{900} / F_{1500}$ with a $15 \%$ uncertainty. Thus, an $\mathrm{S} / \mathrm{N} \approx 20$ is a general guideline to robustly determine the stellar population properties and to infer the ionizing continua.

If the age mixtures were arbitrary mixtures that were not constrained by spectral properties, then we would measure a different fitted age for each iteration, and the inferred age and metallicity uncertainty would be largely unconstrained and uncorrelated with the spectral $\mathrm{S} / \mathrm{N}$. We conclude that the properties of both single-burst and mixed-age populations are relatively robust and constrained by spectral signatures.

\subsection{Mixed-age versus Continuous Star Formation Histories}

In Section 4 we emphasized that the O- and B-star spectral features reflect changes in the inferred stellar population age and metallicity. In Figure 3, we showed that the stellar wind lines are sensitive to the age of the stellar population. Young populations show strong C IV P-Cygni emission, while older stellar populations have more moderate $\mathrm{C}$ IV features. The $\mathrm{N} \mathrm{V}$ wind feature only depends on age (Figure 3), and we see strong galaxy-to-galaxy N V variations (Figure 6). Short-lived WR stars create a broad He II feature that is only seen in the youngest ( $>5 \mathrm{Myr}$ ) spectra (Figure 8). Similarly, older stellar populations have weak, but distinct, photospheric absorption features that are absent in the youngest stellar populations (Figure 9). We concluded that there is real and quantifiable age variation within the observed stellar continua.

The stellar continua vary from galaxy to galaxy, but not always as predicted from a single stellar population. The stellar wind profiles from populations with older light-weighted ages require a mixture of ages (see S1226+2152 in Figure 4). This mixture extends to the ionizing continua, where Figure 17 demonstrated that the inferred ionizing spectra are divided into two star formation histories: single-burst and mixed-age populations. A mixed-age stellar population contains some assortment of young $(<5 \mathrm{Myr})$ and old ( $>8 \mathrm{Myr})$ stellar populations that varies from galaxy to galaxy.

Constant star formation is the canonical alternative to a single-burst star formation history. A constant star formation history assumes that every year a fixed number of stars are formed by consistently populating the IMF. When averaged over a sufficiently long temporal baseline ( $>100 \mathrm{Myr}$, similar to the ages of older B-stars), continuous star formation is a good physical description of the star formation history of evolved star-forming galaxies, such as normal spirals. However, it is unlikely that stars with lifetimes $1 \%-10 \%$ of the dynamical time of galaxies will form at a constant rate. However, in principle, a constant star formation is a singular, simplified mixed-age solution that can be recovered with the proper light fractions.

A constant star formation history has a fixed number of O-stars that produce a fixed number of ionizing photons. The red lines in Figure 13 show the ionizing flux density ratios of a $0.05 Z_{\odot}$ BPASS constant star formation model. This constant star formation law is similar to a mixed-age population with an age of $12 \mathrm{Myr}$ (Figure 17), but a continuous star formation history underpredicts the ionizing continuum of a 8 Myr mixedage population by a factor of 2 , and overpredicts the ionizing continuum of a 25 Myr mixed-age population by a factor of 3 (Figure 17). The number of ionizing photons produced by a constant star formation history only matches the inferred ionizing continua of the mixed-age fits near $12 \mathrm{Myr}$, and a constant star formation history does not match the ionizing continua at other stellar ages.

Only $Z_{*}$ can change the stellar continuum of a constant star formation history. Predominantly age-sensitive tracers, such as $\mathrm{NV}$ and the ionizing continuum, have fixed strengths in a constantly star-forming model. A constant star formation model may fit part of the CIV absorption profile well, but overestimate (or underestimate) the $\mathrm{N} V$ profile because the age has been fixed (see Figure 3). The constant star formation model fails to simultaneously fit the different stellar wind profiles of very young populations (e.g., the Sunburst Arc in Figure 6) or older populations that lack stellar wind signatures (e.g., S1527 +0652 in Figure 6).

Unlike continuous star formation histories, FUV spectral features determine the mixed-age star formation histories and observationally constrain the relative contributions of young and old stars. By accounting for the dominance of young stellar populations to certain ionizing continua, mixed-age star formation histories generate significantly more ionizing 
photons, at higher energies, than continuous star formation histories. The different star formation histories alter the ionizing continua by factors of $2-3$ and must be determined to accurately infer the number of ionizing photons produced by stellar populations.

\subsection{Effect of Binary Evolution on the Stellar Continua}

Most massive stars form in binaries (Sana et al. 2012), and mass can be transferred between the two stars if they are sufficiently close. The star that receives the mass is rejuvenated, and its main-sequence lifetime is extended (Eldridge \& Stanway 2009; Götberg et al. 2017). Mass transfer typically occurs when one star is in an evolved stage and expands to fill the Roche lobe. Consequently, binary star evolution extends the duration that stars spend in late evolutionary phases such as the WR phase (Vanbeveren et al. 2007; Georgy et al. 2012). By increasing the pathways to form evolved stars, populations with binary evolution emit more ionizing photons and have harder ionizing spectra than populations with only single-star evolution (Stanway et al. 2016; Götberg et al. 2017, 2018). Binary evolution establishes a significant evolved population at lower metallicities because lower-metallicity stars are hotter and must expand more in their later evolutionary phases. On the other side of mass transfer, the star that donates its hydrogen envelope is hypothesized to remain as an extremely hot helium core with a blackbody that peaks blueward of $912 \AA$, possibly as an X-ray binary (Van Bever \& Vanbeveren 2000; Mirabel et al. 2011; Fragos et al. 2013; Götberg et al. 2017). These processes add an additional source of extreme ionizing radiation.

Binary star evolution drastically increases the number of ionizing photons produced by a stellar population. Can the restframe FUV observationally constrain the importance of binary evolution? Here we discuss the observational differences between BPASS and STARBURST99 models in their nonionizing spectra (Section 5.4.1) and the differences between the derived stellar population properties (Section 5.4.2), and then we compare the ionizing continua of the two evolution models (Section 5.4.3). We conclude that the non-ionizing continua alone cannot discern the impact of binary evolution, and we explore alternative observational methods to distinguish between binary and single-star models (Section 5.4.4).

\subsubsection{Does Binary Evolution Change the Observed Non-ionizing Stellar Continuum?}

STARBURST99 and BPASS predict comparable non-ionizing continua (see Figure 1). The two use similar O-star libraries and produce largely identical non-ionizing spectral features. The stellar wind shapes (e.g., N V and C IV) slightly differ, but this reflects the subtle differences in the O-star models rather than the effect of binary evolution (Leitherer et al. 2010; Conroy et al. 2014; Eldridge et al. 2017). The S/Ns of individual galaxies are typically too low to distinguish between the two stellar models (compare the blue and gold lines in Figures 4-6). However, the STARBURST99 models fit the N V and C IV wind features of the high-S $/ \mathrm{N}(\mathrm{S} / \mathrm{N}=103$ per resolution element) MEGaSaURA stack marginally better than the BPASS models do (Rigby et al. 2018b).

BPASS and STARBURST99 have significant differences in their older populations. The C III and Si III $1299 \AA$ photospheric features, which we identified in Section 4.2 as strong B-star diagnostics (de Mello et al. 2000), are very weak in the BPASS models (Figure 1). This is likely because BPASS models below $20,000 \mathrm{~K}$ have lower spectral resolution at wavelengths less than $1500 \AA$ and the weak absorption lines are nonphysically blended into the continuum (see the red $15 \mathrm{Myr}$ model in Figure 1). The absence of C III and Si III absorption features is likely due to the inadequate spectral resolution, not binary star evolution.

The broad stellar He II emission is more prominent in 5-8 Myr BPASS models than in STARBURST99 models (Figure 1; Steidel et al. 2016). The BPASS models do fit the He II region substantially better than the STARBURST99 models, but the overall fit is still occasionally poor at young ages (Figure 8). This is likely because binary evolution provides an additional pathway to create and rejuvenate WR stars such that the BPASS models contain more evolved stars, but either the WR evolution or atmosphere models still need refinement. The improved fit of the He II region may indicate that binary stars contribute to the FUV light, but even the BPASS fits do not completely describe the He II region.

Similarly, the increased population of evolved massive stars produces Si IV P-Cygni profiles that are stronger than the $\mathrm{C}$ IV features at ages of 5-10 Myr (Figure 1). Differences between Si IV stellar wind profiles are a key prediction of models that do and do not include binary evolution. Si IV is not prominently observed as a stellar P-Cygni profile at low $Z_{*}$, but Figure 7 shows that the fitted Si IV profiles are statistically similar whether BPASS or STARBURST99 models are used. Even though the single-age BPASS models show broad Si IV P-Cygni profiles, the multiple-age fits do not indicate Si IV P-Cygni features. This is because the population is a mixed-age population with young ( $<5 \mathrm{Myr})$ and old $(>10 \mathrm{Myr})$ populations rather than a single-age $8 \mathrm{Myr}$ population. Thus, the nonionizing stellar continua do not disentangle the effect of binary star evolution on the ionizing continua.

\subsubsection{Does Binary Evolution Affect the Inferred Light-weighted Stellar Ages and Metallicities?}

The light-weighted metallicities and ages derived with BPASS and STARBURST99 in Figure 18 are similar. However, BPASS models have a lower spectral resolution at wavelengths $<1500 \AA$ for stellar temperatures $<25,000 \mathrm{~K}$. This means that the BPASS models do not have a single spectral resolution over the bandpass. This biases the COS fits either toward younger stellar populations, where the spectral resolution matches the data, or toward older populations, where multiple individual features are combined into single unresolved features. These resultant BPASS fits to the COS data often do not match the $\mathrm{N} \mathrm{V}$ stellar wind profiles. We tested that the reduced wavelength coverage causes the poor fitting of the COS data by fitting the MEGaSaURA data with only rest-frame wavelengths $<1500 \AA$. We found that the BPASS fits to the MEGaSaURA data with only wavelengths $<1500 \AA$ were similarly poorly fit by the BPASS models, but the STARBURST99 fits were not affected by using the restricted wavelength regime. Consequently, we only compare the BPASS fits for the MEGaSaURA data, which have a complete wavelength coverage.

The inferred light-weighted stellar metallicities (at $3 \sigma$ significance) and ages ( $5 \sigma$ significance) scale along the oneto-one relationship in Figure 18. The ages are similar for young populations $(<15 \mathrm{Myr})$, while BPASS models systematically 

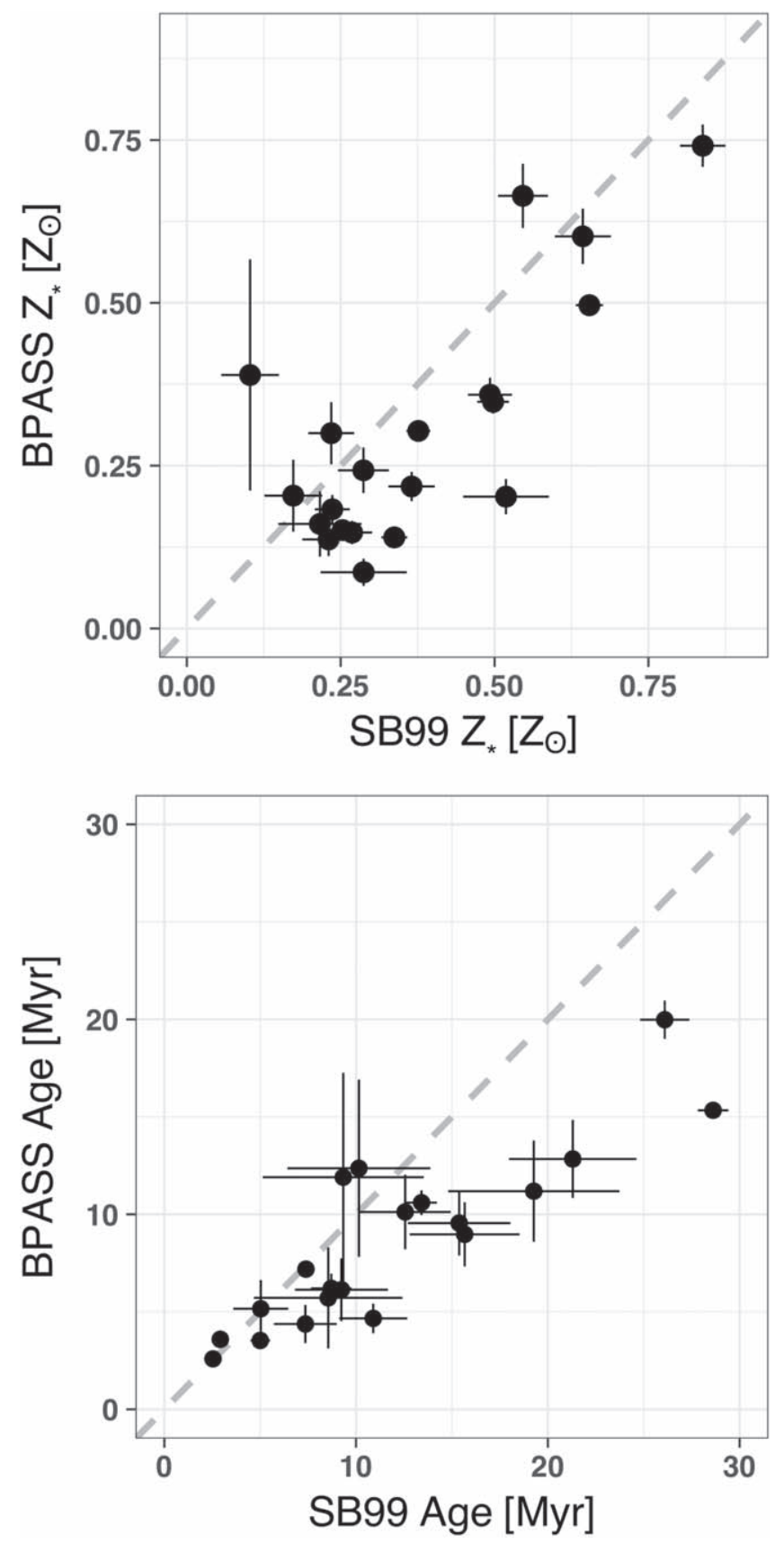

Figure 18. Comparison of the derived stellar population properties of the MEGaSaURA sample computed using a linear combination of either STARBURST99 (SB99; $x$ axis) or BPASS ( $y$ axis) stellar models. Top panel: comparison of the light-weighted stellar metallicities $\left(Z_{*}\right) . Z_{*}$ generally follows the one-to-one trend (gray dashed line). Bottom panel: comparison of the lightweighted stellar age. There is a slope change near $15 \mathrm{Myr}$ largely because high spectral resolution BPASS stellar templates do not exist at all wavelengths past this age (Figure 1). Consequently, the oldest inferred BPASS ages are younger than the inferred STARBURST99 ages. Even still, there is a qualitative agreement such that old inferred BPASS populations are also old STARBURST99 populations.

estimate younger ages when STARBURST99 ages are $>15$ Myr. This split happens at the same age as the degradation of the BPASS spectral resolution discussed above. Thus, we conclude that, while the poor BPASS spectral resolution inhibits a firm conclusion, the ages and $Z_{*}$ derived using the different stellar models are similar. In the next section, we compare the BPASS ionizing continua, but we note that the inferred BPASS ages are up to $30 \%$ younger for the oldest populations.

\subsubsection{Binary Evolution Strongly Affects the Ionizing Continua}

The CIV (Figures 4 and 5) and NV (Figure 6) regions cannot discriminate between binary and single-star populations. While BPASS models fit the He II region better than STARBURST99 models (Figure 8), neither successfully reproduces the full He II spectral shape. We concluded that the fitted nonionizing continua cannot determine the contribution of binary star evolution to the stellar continuum.

Conversely, the BPASS-modeled ionizing continua drastically differ from the STARBURST99 continua. This is most extreme for older and lower-metallicity stellar populations (Figures 13 and 14). At young ages ( $<3 \mathrm{Myr})$, the production of ionizing photons is similar regardless of the binary evolution model because young stars have not yet evolved off the main sequence. Meanwhile, at older ages, such as $8 \mathrm{Myr}$, there is a factor of 2 difference in $F_{900} / F_{1500}$ between the BPASS and STARBURST99 models. This difference is similar to the difference between a burst and an $8 \mathrm{Myr}$ mixed-age population (Section 5.2.2).

The modeled ionizing continua differ most drastically at higher energies (right two panels of Figure 13). A single $10 \mathrm{Myr}$ STARBURST99 burst has negligible $F_{300} / F_{1500}=$ $3 \times 10^{-8}$, but a population that includes binary evolution produces a staggering $F_{300} / F_{1500}=0.20$ (the circle farthest right in the right panel of Figure 12). Populations with binary evolution have substantially harder ionizing spectra, due to the enhanced contribution of evolved stars.

Thus, the inferred BPASS ionizing continua are typically stronger and harder than those inferred from STARBURST99 (blue versus gold curves in Figure 16). While the BPASS models exhibit the same qualitative trend of decreasing $F_{900} / F_{1500}$ with inferred stellar age, there are important differences between the ionizing continua inferred with BPASS and STARBURST99. RCS Knot E (top panel) has the youngest MEGaSaURA population (2.5 Myr), and the inferred ionizing continuum is similar for both the STARBURST99 and BPASS models. Conversely, the Sunburst Arc, which is only $0.5 \mathrm{Myr}$ older, has similar $F_{900} / F_{1500}$ values, but the $F_{525} / F_{1500}$ and $F_{300} / F_{1500}$ are 1.4 and 4.9 times larger for the BPASS models than for the STARBURST99 models, respectively. The oldest galaxy, S1226+2152, again has the lowest $F_{900} / F_{1500}$, but $F_{900} / F_{1500}, F_{525} / F_{1500}$, and $F_{300} / F_{1500}$ are $1.4,2.0$, and 7.2 times larger for the BPASS models than the STARBURST99 models.

S0108+0624 has the most pronounced difference in the BPASS and STARBURST99 ionizing continua. The inferred $F_{900} / F_{1500}, F_{525} / F_{1500}$, and $F_{300} / F_{1500}$ are 2 , 4 , and 12 times larger for the BPASS fits than STARBURST99. Surprisingly, the BPASS models in Figure 16 predict larger $F_{300} / F_{1500}$ for the older population of $\mathrm{S} 0108+0624$ than the youngest stellar populations in the sample, such as RCS Knot E (1.4 times larger) and the Sunburst Arc (1.7 times larger).

The inferred ionizing continua of stellar populations that include binary evolution can produce up to 12 times more hard ionizing photons per FUV luminosity than single-star populations with the same FUV spectral features. This presents an uncomfortable conclusion: there is up to an order of magnitude difference in the number of ionizing photons predicted from populations with binary evolution that cannot be observationally 
constrained by the non-ionizing stellar continuum. Additional observations must constrain the effect of binary star evolution.

\subsubsection{How to Observationally Distinguish Binary and Single-star Populations}

The previous subsections demonstrated that the non-ionizing stellar continua cannot quantify the effect of binary star evolution on the integrated stellar spectra, but there are extreme differences in their respective ionizing continua. While observations suggest that massive stars typically form in binaries, the exact binary parameters-mass ratios, period distributions, and remnant masses-are largely unknown and must be assumed to create the BPASS models (Eldridge et al. 2017). Therefore, the effect of binary evolution on the stellar continuum is observationally unconstrained. Observational tests must break this degeneracy.

Direct observations of the extreme-UV continuum (EUV; wavelengths $<912 \AA$ ) are a definitive method to break this degeneracy. With up to a factor of 12 flux density difference between the BPASS and STARBURST99 modeled ionizing continua, direct observations would indisputably determine the effect of binary star evolution on the ionizing continuum. Further, there are strong stellar wind lines in the EUV (e.g., $\mathrm{N}$ III $763 \AA$ ) that are more prevalent in binary populations than in single-star populations (Figure 16). The intrinsic LyC break (near $912 \AA$ ) is also different for 5-10 Myr populations, due to the higher stellar temperatures of binary populations (Figure 12). However, the EUV of an O-star-dominated stellar population has never been observed.

Steidel et al. (2016) broke this degeneracy using nebular emission lines. For an ionization-bounded nebula (or one where the gas absorbs all of the ionizing photons), the relative strength of nebular emission lines at different ionization states (e.g., [O III] $5007 \AA$ versus [O II] $3727 \AA$ ) traces the intrinsic hardness of the stellar spectrum, and $\mathrm{H}$ and $\mathrm{He}$ recombination lines trace the intensity (normalization) of the ionizing continuum (modulo the escape of ionizing photons and the effects of dust). Constraining whether the inferred BPASS or STARBURST99 ionizing continua better reproduce the observed nebular emission line structure will illuminate the importance of binary evolution.

Comparing [O III] and [O II] emission lines may resolve the different ionizing continua of the two evolutionary models. BPASS models create more $\mathrm{O}^{++}$gas relative to low-ionization, $\mathrm{O}^{+}$, gas than single-star models (Stanway et al. 2014). We illustrate this by comparing the $F_{300} / F_{900}$ ratios of the STARBURST99 (blue lines in Figure 19) and BPASS models (gold lines in Figure 19). Photons at $300 \AA$ produce $\mathrm{O}^{++}$gas, while photons at $900 \AA$ only generate $\mathrm{O}^{+} ; F_{300} / F_{900}$ probes the formation of $\mathrm{O}^{++}$and $\mathrm{O}^{+}$gas, which emit [O III] and [O II] , respectively. This is meant to illustrate the possibility of observing differences in the $[\mathrm{O} \mathrm{III}] /[\mathrm{O} \mathrm{II}]$ ratio with stellar age, but full photoionization modeling is required to compare to the observed emission lines. Models without binary evolution produce $\mathrm{O}^{++}$at low metallicities and young ages, while BPASS models produce large $X\left(\mathrm{O}^{++}\right) / X\left(\mathrm{O}^{+}\right)$ratios at all metallicities and ages. In fact, high- $Z_{*}$ BPASS models produce larger $X\left(\mathrm{O}^{++}\right) / X\left(\mathrm{O}^{+}\right)$ratios than the youngest low-metallicity singlestar models. Correlations between $X\left(\mathrm{O}^{++}\right) / X\left(\mathrm{O}^{+}\right)$and stellar population properties may constrain the importance of binary evolution.

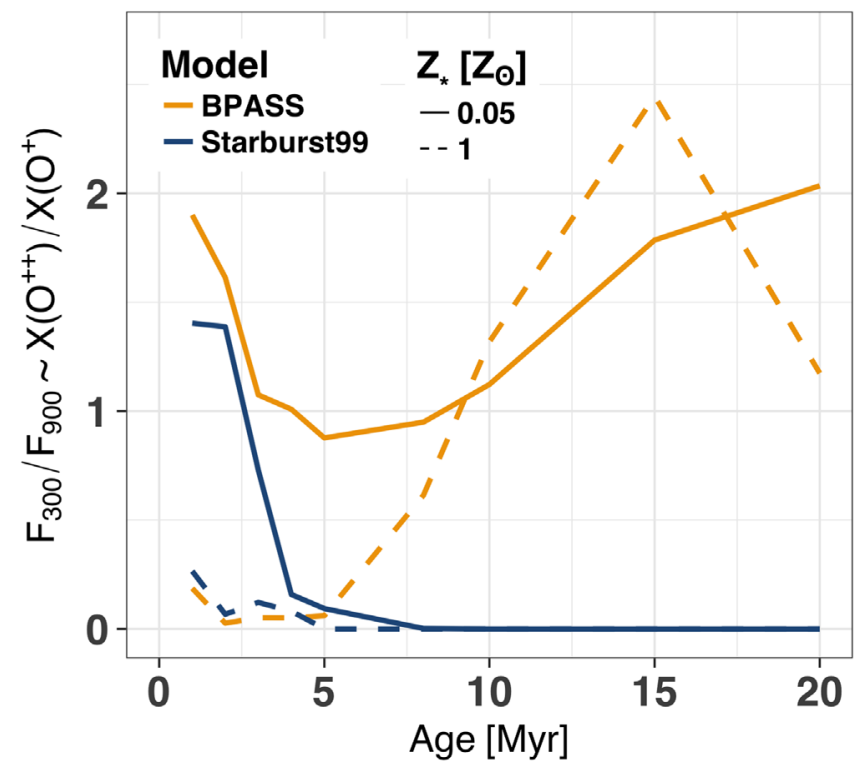

Figure 19. Age variation of the theoretical stellar continuum flux density ratio at $300 \AA\left(F_{300}\right)$ and $900 \AA\left(F_{900}\right)$ for STARBURST99 (gold lines) and BPASS (blue line) single-burst models. Solid lines are $0.05 Z_{\odot}$ stellar metallicities, and dashed lines are $1 Z_{\odot}$ stellar metallicities. Photons at $300 \AA$ create $\mathrm{O}^{++}$and photons at $900 \AA$ create $\mathrm{O}^{+}$, so the $F_{300} / F_{900}$ ratio probes the ionization fraction $X\left(\mathrm{O}^{++}\right) /$ $X\left(\mathrm{O}^{+}\right)$. Full photoionization modeling is required to explore the actual nebular emission line ratios ([O III]/[O II $]$ ). STARBURST99 models only produce extreme amounts of hard ionizing photons within the first $5 \mathrm{Myr}$ and at low metallicities. BPASS models produce hard ionizing photons at later ages and all metallicities. Older, higher metallicity BPASS models actually produce relatively more highenergy photons than younger, metal-poor populations.

Individual emission lines may also break the degeneracy between binary and single-star models. Nebular He II emission requires extremely high-energy photons $(\lambda<227 \AA)$. Stellar continua are typically considered too weak and too soft to reproduce the observed He II $1640 \AA$ and He II $4686 \AA$ nebular emission (Kehrig et al. 2015; Berg et al. 2018; Nanayakkara et al. 2018; Stanway \& Eldridge 2018a; Schaerer et al. 2019). S0108+0624 is the only spectrum in the MEGaSaURA sample with a weak and narrow He II emission line (Figure 8; equivalent width of $-0.3 \pm 0.1 \AA$ ). Figure 16 shows that the BPASS fit to S0108+0624 has a harder spectrum than the youngest stellar populations (compare the gold and gray lines in the third panel), possibly suggesting that evolved, mixed-age binary star populations are required to produce the observed nebular He II.

\subsection{Fits to Stacked MEGaSaURA Spectra}

Stacking, or averaging many spectra together, increases the $\mathrm{S} / \mathrm{N}$ of the composite spectrum. Often it is assumed that a stacked spectrum probes the average properties of the underlying population. The combination of the high-quality individual MEGaSaURA spectra and the stacked MEGaSaURA spectrum allows us to test this hypothesis.

By weighting the individually measured stellar ages and metallicities by the $\mathrm{S} / \mathrm{N}$ of their respective MEGaSaURA spectrum at $1500 \AA$, we estimated the stellar age and metallicity of the MEGaSaURA ensemble to be $14.0 \pm 1.3 \mathrm{Myr}$ and $0.47 \pm$ $0.03 Z_{\odot}\left(9.7 \pm 1.0 \mathrm{Myr}\right.$ and $0.35 \pm 0.02 Z_{\odot}$ for BPASS $)$. We then fit the stacked MEGaSaURA spectrum and estimated a lightweighted age and metallicity of $12.0 \pm 0.7 \mathrm{Myr}$ and $0.42 \pm$ $0.01 Z_{\odot}\left(9.9 \pm 0.6 \mathrm{Myr}\right.$ and $0.27 \pm 0.01 Z_{\odot}$ for BPASS $)$. Thus 
the inferred stellar properties of the stack are statistically similar to the ensemble averages.

The $\mathrm{S} / \mathrm{N}$ weighted average $F_{900} / F_{1500}$ of the individual fits is $0.68 \pm 0.42$, and the inferred $F_{900} / F_{1500}$ from the stack is 0.47. Meanwhile, if we put the fitted light-weighted age of the stellar population into the $F_{900} / F_{1500}$ relationship (Equation (6)), we expect $F_{900} / F_{1500}=0.77$. We find that the fitted stellar properties of the MEGaSaURA stacked spectra reasonably match the underlying population averages.

\subsection{Comparison to Previous Work}

The work most directly comparable to our stellar population synthesis fitting is Steidel et al. (2016). Those authors used a stack of 30 galaxies at $z \sim 2.4$ with rest-frame FUV and optical observations (for the optical emission lines) to determine the stellar population and nebular emission properties. They fit a variety of $Z_{*}$, IMFs, and stellar models (BPASS versus STARBURST99) to the observed stellar continuum, but always used a continuous star formation history. Low-metallicity $\left(\sim 0.05 Z_{\odot}\right)$ continuous star formation models fit the stellar continuum best, even though the measured nebular metallicities were $Z_{\text {neb }}=0.29-0.49 Z_{\odot}$.

The various continuous star formation stellar continuum models were then used as the ionizing source within CLOUDY photoionization models to predict the nebular emission lines and compare these predictions to the observed values. Continuous star formation STARBURST99 models produced an insufficient number of ionizing photons to match the observed classical Baldwin et al. (1981) diagnostics such as [O III] $5007 \AA / \mathrm{H} \beta$ and [N II] $6585 \AA / \mathrm{H} \alpha$. Similarly, BPASS models with $Z_{*}=Z_{\text {neb }}$ failed to reproduce these nebular emission-line ratios.

However, Steidel et al. (2016) could reproduce the emission lines if $Z_{*}$ was five times smaller than $Z_{\text {neb }}$. Steidel et al. (2016) argued that rather than a lower $Z_{*}$, the $\alpha /$ Fe relative abundance ratio of $z \sim 2$ galaxies must be supersolar. This would lead to a measured $Z_{*}$ that is smaller than the actual metallicity, and the stars would produce more ionizing photons because $\mathrm{Fe}$ is the chief opacity source in the atmospheres of massive stars. Thus, they concluded that the nonsolar $\alpha / \mathrm{Fe}$ relative abundances produced an illusion that the stars had five times fewer metals than the gas.

We fit the Steidel et al. (2016) stack with the methods outlined above and derived a light-weighted $Z_{*}=0.37 \pm 0.04 Z_{\odot}$ using STARBURST 99 models $\left(0.22 \pm 0.02 Z_{\odot}\right.$ using BPASS $)$. The inferred STARBURST99 stellar population age of the Steidel et al. (2016) stack is $12.6 \mathrm{Myr}$, implying that it is a mixed-age population. These metallicities are similar to the nebular metallicities of $Z_{\mathrm{neb}}=0.29-0.49$ calculated using the direct and strong-line methods (Steidel et al. 2016; Strom et al. 2017, 2018), implying that the stars and gas have similar metallicities (we plot both the direct and strong-line $Z_{\text {neb }}$ calculations in Figure 11).

While the inferred light-weighted $Z_{*}$ is meant to convey a total metal content, the strong stellar wind lines, which are only observed through $\alpha$-element transitions in the FUV, could hide discrepant $\alpha / \mathrm{Fe}$ abundance ratios (Steidel et al. 2016). Therefore, Steidel et al. (2016) suggested using regions devoid of strong wind features but containing many weak photospheric lines (the so-called "featureless" regions in Table 2) to test the stellar Fe abundance. Using "Mask 2" from Steidel et al. (2016), we refit their composite spectra using only wavelengths

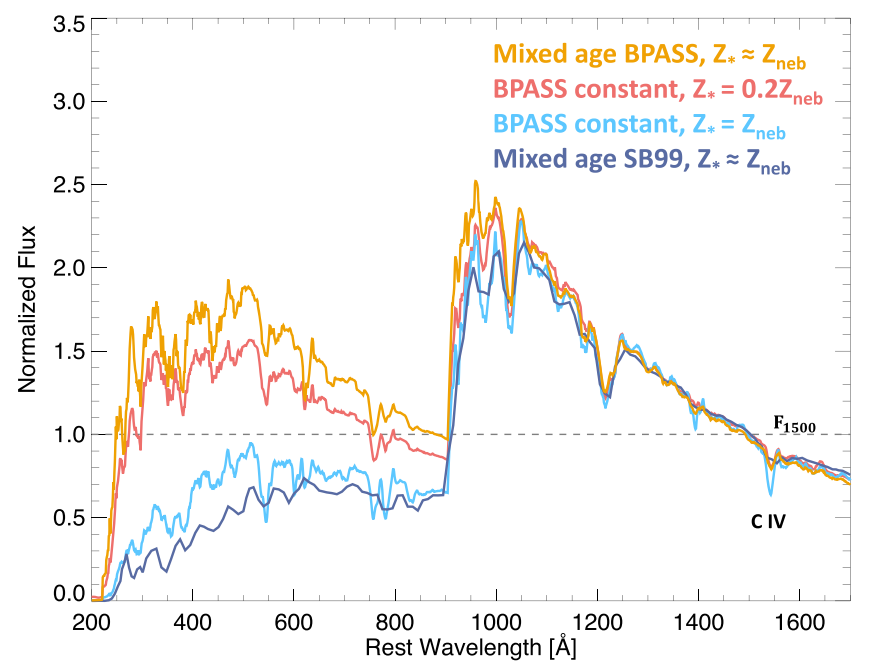

Figure 20. Comparison of the inferred stellar continua of the stacked spectrum from Steidel et al. (2016) using a linear combination of single-age BPASS (in orange with a light-weighted age of $10 \mathrm{Myr}$ and $0.22 Z_{\odot}$ ) and STARBURST99 (dark blue with a light-weighted age and metallicity of $13 \mathrm{Myr}$ and $0.37 Z_{\odot}$ ). The $0.05 Z_{\odot}\left(1 / 5 \times Z_{\text {neb }}\right)$ constant star formation model that reproduces the nebular emission structure is shown in red, while the constant star formation model with $Z_{*}=Z_{\text {neb}}$, which does not reproduce the nebular emission lines, is in light blue. The constant and mixed-age BPASS models have similar shapes, even though their metallicities differ by a factor of 4 . The light-weighted $Z_{*}$ of the mixed-age fits are similar to the measured nebular metallicity of 0.29-0.49 $Z_{\odot}$ (Steidel et al. 2016). Mixed-age populations produce a strong and hard ionizing continuum, while having a metallicity similar to the nebular gas.

without strong stellar wind lines and find a very similar lightweighted $Z_{*}$ as when we fit the entire spectrum $(0.43$ and $0.37 Z_{\odot}$ for STARBURST99 and BPASS, respectively). Thus, even in stellar spectral regions dominated by $\mathrm{Fe}$ absorption features, we still infer that $Z_{*}$ and $Z_{\text {neb }}$ are similar.

The only difference between the inferred $Z_{*}$ from this work and Steidel et al. (2016) is the assumed star formation history. As emphasized in Section 5.3, a constant star formation history predetermines a fixed number of young and older stars, each with their own distinct spectral signatures. Because young populations have Fe IV and Fe V photospheric lines (Figure 1) and slightly older populations generally have Fe III lines (Figure 5 in de Mello et al. 2000), a continuously star-forming population is always expected to have Fe III, Fe IV, and Fe V absorption. The assumption of constant star formation may force the Steidel et al. (2016) fits toward lower metallicities in order to balance the strength of the Fe absorption across different ionization states. By contrast, a mixed-age stellar population can reduce the $\mathrm{Fe}$ absorption lines by either reducing the metallicity or by selecting a combination of stellar population ages to match the observed Fe lines (i.e., an older population without $\mathrm{Fe}$ IV and $\mathrm{FeV}$ or a younger population without Fe III). Thus, we emphasize that the a priori assumed star formation history (constant versus mixed age) has a profound impact on the inferred stellar metallicity.

Even though the light-weighted $Z_{*}$ derived from our mixedage fits to the stellar continuum are four times larger, the C IV $1550 \AA$ stellar wind P-Cygni profile appears similar to the lower-metallicity, $0.05 Z_{\odot}$ continuous star formation model (orange and red lines Figure 20) and weaker than the $0.3 Z_{\odot}$ constant star formation model (light blue line). As discussed in Section 5.3, continuous star formation models remove possible 
age variations, leaving $Z_{*}$ as the only lever to influence the C IV P-Cygni shape. The mixed-age fits to the stacked data have light-weighted ages of $12.6 \pm 2.4 \mathrm{Myr}$ using STARBURST99 (10.1 $\pm 1.9 \mathrm{Myr}$ using BPASS $)$ such that a moderate-metallicity, moderate-age population has relatively weak C IV absorption.

Figure 20 shows the inferred ionizing continuum from the fit to the Steidel et al. (2016) stack. This figure strongly emphasizes that mixed-age populations produce substantially more ionizing photons, even at higher metallicities, than continuous star formation models. The STARBURST99 fit (dark blue line) has a weaker ionizing continuum than the BPASS fit (orange line), and the STARBURST99 fit is more similar to the BPASS $Z_{*}=Z_{\text {neb }}$ continuous star formation model (light blue), which failed to reproduce the nebular emission. As suggested by Steidel et al. (2016), the STARBURST99 fits are unlikely to generate sufficient ionizing photons to match the nebular emission.

The BPASS mixed-age fit to the stack (orange curve in Figure 20) produces more ionizing photons, with a similar spectral shape, than the low-metallicity continuous star formation model, which reproduced the nebular emission structure (red curve). The youngest stellar populations produce the most ionizing photons (Figure 15), so allowing younger populations to have larger light fractions increases the total number of ionizing photons produced by a stellar population (see Section 5.7). Consequently, a mixed-age stellar population produces more ionizing photons than a continuous star-forming model, while having a similar stellar and nebular metallicity.

\subsection{Production Efficiency of Ionizing Photons by Massive Star Populations}

Throughout this paper, we have described the ionizing continua using $F_{900} / F_{1500}$, a measure of the ionizing continuum at a single wavelength. However, the principal goal of this paper is to use the FUV stellar continua to determine a more physically important parameter: the total number of ionizing photons produced by massive star populations $(Q)$. Note that $Q$ scales linearly with the star formation rate, such that populations forming more stars generate more ionizing photons (Kennicutt 1998; Madau et al. 1998; Kennicutt \& Evans 2012; Madau \& Dickinson 2014). Therefore, the ionizing photon production efficiency, $\xi_{\text {ion }}$, compares the capacity of different stellar populations to produce ionizing photons at a given FUV luminosity. The ionizing photon production efficiency is defined from the stellar models as

$$
\xi_{\text {ion }}\left[\text { photons } \AA \mathrm{erg}^{-1}\right]=\frac{Q\left[\text { photons s}{ }^{-1}\right]}{L_{1500}\left[\operatorname{erg} \AA^{-1} \mathrm{~s}^{-1}\right]},
$$

or in the more common units as

$$
\xi_{\text {ion }}\left[\text { photon } \mathrm{Hz} \mathrm{erg}^{-1}\right]=\frac{Q\left[\text { photon } \mathrm{s}^{-1}\right]}{L_{1500}\left[\mathrm{erg}_{\mathrm{Hz}}^{-1} \mathrm{~s}^{-1}\right]} .
$$

When multiplied by the reddening-corrected FUV luminosity, $L_{1500}, \xi_{\text {ion }}$ determines the total number of ionizing photons generated by a stellar population.

Figure 21 shows that the stellar population parameters, particularly stellar age, determine $\xi_{\text {ion. }}$ A $2 \mathrm{Myr}, 0.4 Z_{\odot}$ STARBURST99 stellar population produces 50 times more ionizing photons per $L_{1500}$ than a similar $10 \mathrm{Myr}$ population. Similarly, a $0.05 Z_{\odot}, 2 \mathrm{Myr}$ population produces three times

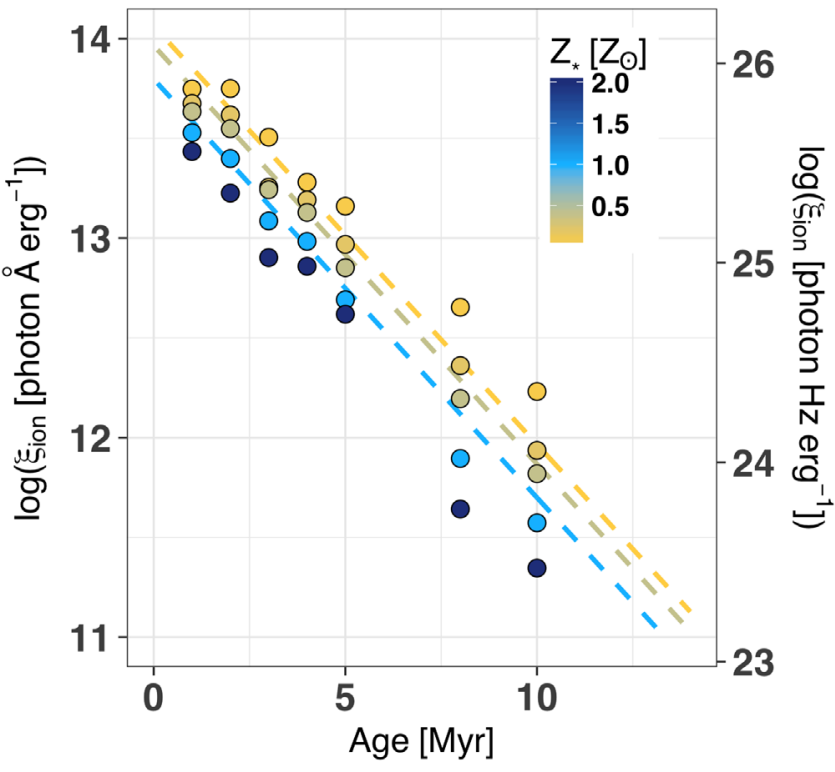

Figure 21. Scaling of the ionizing photon production efficiency ( $\xi_{\text {ion }}$; the number of ionizing photons per FUV luminosity) for a single-burst STARBURST99 stellar population with age ( $x$ axis) and metallicity $\left(Z_{*}\right.$; color bar) Younger stellar populations produce an order of magnitude more ionizing photons per FUV luminosity than older stellar populations. The blue, tan, and gold curves are single-metallicity relations for $\xi_{\text {ion }}$ using Equation (10) with $Z_{*}$ of $1,0.4$, and $0.05 Z_{\odot}$, respectively.

more ionizing photons than a $2 Z_{\odot}$ population of the same age. These numbers stress the relative importance of age for the production of ionizing photons. The multivariate relationship between $\xi_{\text {ion }}$ and the STARBURST99 stellar population properties is

$$
\xi_{\text {ion }}\left[\text { photon } \AA \mathrm{erg}^{-1}\right]=\left(1.2 \times 10^{14}\right) 10^{-0.21 \frac{\mathrm{Age}}{1 \mathrm{Myr}}-0.28 \frac{Z_{*}}{1 Z_{\odot}},}
$$

or

$$
\xi_{\text {ion }}\left[\text { photon } \mathrm{Hz} \mathrm{erg}^{-1}\right]=\left(1.6 \times 10^{26}\right) 10^{-0.21 \frac{\mathrm{Age}}{1 \mathrm{Myr}}-0.28 \frac{Z_{*}}{1 Z_{\odot}} .}
$$

This relation powerfully illustrates that the total number of ionizing photons, $Q$, can be determined from the stellar population age, metallicity, and the extinction-corrected $L_{1500}$. As with the $F_{900} / F_{1500}$ relations, the models vary from the fitted relation at a given age (the curves in Figure 21 are singlemetallicity relations), but Equations (10) and (11) characterize the overall evolution of the efficiency of ionizing photon production with an observed stellar age and metallicity.

Equations (10) and (11) have exceptionally similar scalings with age and $Z_{*}$ to $F_{900} / F_{1500}$ as found in Figure 15 . In fact, $F_{900} / F_{1500}$ strongly scales with $\xi_{\text {ion }}$ (Figure 22) as

$$
\log \left(\xi_{\text {ion }}\left[\AA \mathrm{erg}^{-1}\right]\right)=13.23+1.23 \times \log \left(\frac{F_{900}}{F_{1500}}\right) .
$$

The strong relationship between $F_{900} / F_{1500}$ and $\xi_{\text {ion }}$ may come as a surprise, but it is simply related by changes in stellar temperature. As the stellar temperature decreases with increasing stellar age, the hydrogen within the stellar atmospheres becomes more neutral and the stellar Lyman break increases 


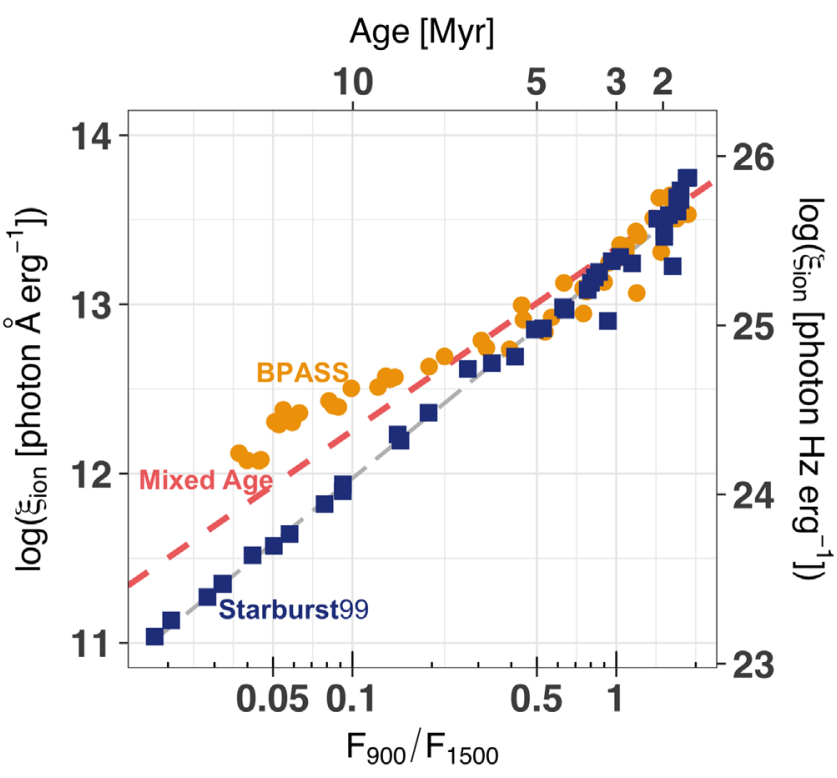

Figure 22. Intrinsic number of ionizing photons per FUV luminosity generated by a theoretical stellar population $\left(\xi_{\text {ion }}\right)$ vs. the ratio of the flux density at $900 \AA$ to the flux density at $1500 \AA\left(F_{900} / F_{1500}\right)$. The blue points are single-burst STARBURST99 populations, and the gray line is the best-fit relation to these points (Equation (12)). The relation found from the MCMC STARBURST99 mixed-age population is shown by the red line (Equation (14)). Models that include binary evolution are gold circles. The upper $x$ axis shows the stellar age of a $0.4 Z_{\odot}$ model: at young ages $(<5 \mathrm{Myr})$, all models produce a similar number of ionizing photons per FUV luminosity.

(compare the $2 \mathrm{Myr}$ to the 3 and $5 \mathrm{Myr}$ models in Figure 12). Thus, $F_{900} / F_{1500}$ effectively measures the hydrogen ionization fraction within the stellar atmosphere, which is related to the stellar temperature by both the stellar age and metallicity. Since stars are to first order complicated blackbodies, it follows that the integrated ionizing energy emitted by massive stars, $\xi_{\text {ion }}$, must strongly scale with the stellar temperature (or proxies thereof, such as $\left.F_{900} / F_{1500}\right)$ through the Stephan-Boltzmann law. Thus, the analysis of the variation of $F_{900} / F_{1500}$ with age and $Z_{*}$ from Section 5.2.1 directly extends to $\xi_{\text {ion }}$.

Populations with binary evolution produce a flatter relationship between $\xi_{\text {ion }}$ and $F_{900} / F_{1500}$ (orange circles in Figure 22), because binary populations produce more evolved stars, which in turn have higher temperatures at older ages. Thus, $\xi_{\text {ion }}$ from BPASS models scales as

$$
\log \left(\xi_{\text {ion }}\left[\AA \mathrm{erg}^{-1}\right]\right)=13.27+0.82 \times \log \left(\frac{F_{900}}{F_{1500}}\right) .
$$

At $F_{900} / F_{1500}=0.1$ (or an age of $\sim 10 \mathrm{Myr}$ ), populations with binary evolution produce three times more total ionizing photons than populations without binary evolution. However, at ages $<5 \mathrm{Myr}$, both models produce a similar number of ionizing photons. Thus, binary evolution increases the total number of ionizing photons produced by older stellar populations, but has minimal impact on the youngest, 2-4 Myr stellar populations.

Similarly, mixed-age stellar populations produce more ionizing photons per $L_{1500}$ at older light-weighted ages than a single-burst star formation history (red line in Figure 22), due to the outsized contribution of younger populations (Section 5.3). Using the same MCMC sample as Section 5.2.2, there is a strong

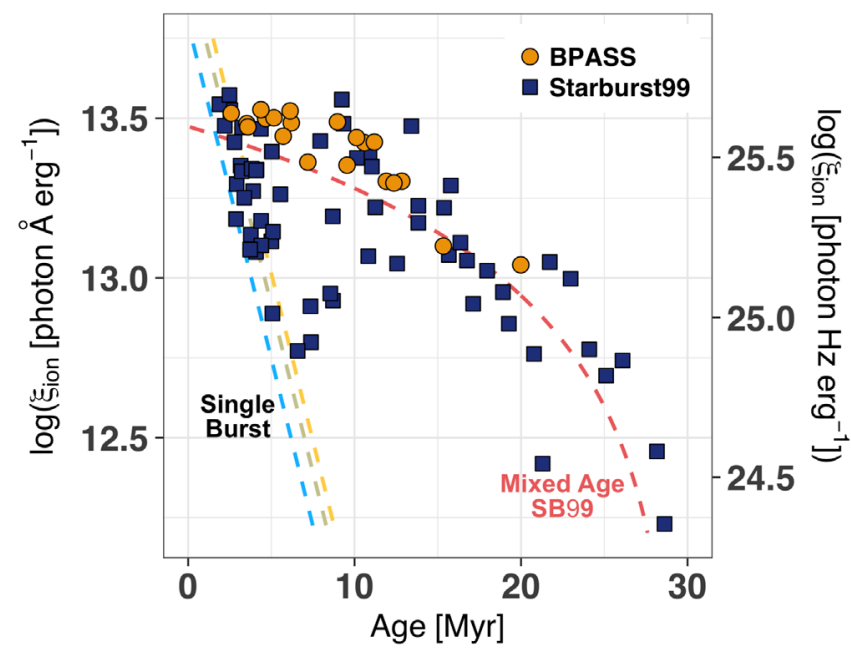

Figure 23. Inferred ionizing photon production efficiency $\left(\xi_{\text {ion }}\right)$ from integrating the ionizing continuum of the stellar continuum fits vs. the inferred light-weighted stellar age using the STARBURST99 (blue squares) and BPASS models (orange circles). Only the MEGaSaURA spectra were fit with BPASS models, due to the resolution discrepancy. The blue, tan, and gold curves are single-burst $Z_{*}$ STARBURST99 curves (Equation (10)) with $Z_{*}$ of $1,0.4$, and $0.05 Z_{\odot}$, respectively. The red dashed line is the relationship from the MCMC STARBURST99 mixed-age models.

correlation between $\xi_{\text {ion }}$ and $F_{900} / F_{1500}$ for the mixed-age STARBURST99 population:

$$
\log \left(\xi_{\text {ion }}\left[\AA \mathrm{erg}^{-1}\right]\right)=13.33+1.08 \times \log \left(\frac{F_{900}}{F_{1500}}\right) .
$$

These three cases illustrate that at older light-weighted ages $\xi_{\text {ion }}$ strongly depends on both the star formation history and the effect of binary evolution.

Finally, we compare the ionizing photon production efficiencies of the observed stellar spectra in Figure 23. The $\xi_{\text {ion }}$ values are determined by dividing the modeled continuum by the photon energy and integrating the entire ionizing continuum spectrum. Galaxies that we defined as having a single-burst star formation history in Section 5.2.2 closely track the single-burst $\xi_{\text {ion }}$ relations of Equation (10) (blue, tan, and yellow curves), while mixed-age populations follow the curve defined by the synthetic MCMC population (red curve).

The median $\log \left(\xi_{\text {ion }}\left[\AA \mathrm{Arg}^{-1}\right]\right)$ of the observed sample using the STARBURST99 models is $13.2 \pm 0.2(25.3 \pm 0.2$ for $\left.\log \left(\xi_{\text {ion }}\left[\mathrm{Hz} \mathrm{erg}^{-1}\right]\right)\right)$, and the full $\log \left(\xi_{\text {ion }}\left[\AA_{\mathrm{erg}}^{-1}\right]\right)$ range is 12.2-13.6 (24.4-25.7 for $\left.\log \left(\xi_{\text {ion }}\left[\mathrm{Hz} \mathrm{erg}^{-1}\right]\right)\right)$. The galaxies within our sample have over an order of magnitude range in the number of ionizing photons that high-mass stars produce per FUV luminosity. Note that $\xi_{\text {ion }}$ strongly varies from galaxy to galaxy; accurate stellar continuum modeling must determine how efficiently ionizing photons are produced. Perhaps most importantly, the range of inferred $\xi_{\text {ion }}$ shows that the number of ionizing photons cannot be estimated from the observed FUV luminosity to better than an order of magnitude without estimating the stellar population age and metallicity.

The measured $\xi_{\text {ion }}$ values are largely consistent with values inferred in the literature. Kennicutt \& Evans (2012) used a constant star formation history, $1 Z_{\odot}$ STARBURST99 model to derive expressions for the star formation rate of normal local galaxies. These models have $\log \left(\xi_{\text {ion }}\left[\AA \mathrm{erg}^{-1}\right]\right)=13.0$ $\left(\log \left(\xi_{\text {ion }}\left[\mathrm{Hz} \mathrm{erg}^{-1}\right]\right)=25.1\right)$, which is statistically consistent with the median value of our sample. While this assumed $\xi_{\text {ion }}$ 
value agrees with the median of our sample, it will not agree on a galaxy-by-galaxy basis, for which a detailed analysis of the massive star properties is required. At higher redshifts, it is typically presumed that more extreme objects, with $\log \left(\xi_{\text {ion }}\left[\mathrm{Hz} \mathrm{erg}^{-1}\right]\right)>25.2-25.3$, are required to reionize the early universe if $20 \%$ of their ionizing photons escape the interstellar medium (Robertson et al. 2013; Bouwens et al. 2016; Finkelstein et al. 2019). Half of our sample, 31 of 61 $(51 \%)$, have $\xi_{\text {ion }}$ greater than the value typically assumed for cosmic reionization $\left(\log \left(\xi_{\text {ion }}\left[\mathrm{Hz} \mathrm{erg}^{-1}\right]\right)>25.3\right)$. This is not surprising because the galaxies were selected to be bright in the rest-frame FUV, such that the stars must be relatively young. Finally, local galaxies that have been observed to emit ionizing photons also have very young stellar populations (Izotov et al. 2018) and correspondingly extreme values of $\xi_{\text {ion }}\left[\mathrm{Hz} \mathrm{erg}^{-1}\right]=25.6$ measured from their C III $] 1909 \AA$ emission lines (Schaerer et al. 2018). These $\xi_{\text {ion }}$ values are consistent with the youngest 2-3 Myr stellar populations in both of our samples.

The BPASS fits to the MEGaSaURA spectra indicate that binary evolution increases the duration of the peak $\xi_{\text {ion }}$, but not the peak $\xi_{\text {ion }}$ itself (orange circles versus blue squares in Figure 23). At the youngest ages, the inferred $\xi_{\text {ion }}$ values from the STARBURST99 and BPASS fits are very similar; binary evolution does not increase the maximum inferred $\xi_{\text {ion }}$. Consequently, the youngest stellar populations, which produce the majority of the ionizing photons, have consistent $\xi_{\text {ion }}$ values regardless of the chosen binary evolution model. Thus, binary evolution does not increase the maximum $\xi_{\text {ion }}$; rather, it elongates the period over which stellar populations produce their maximum $\xi_{\text {ion }}$. If binary evolution is important at high redshifts, it would increase the total number of ionizing photons that high-mass stellar populations produced over their entire lifetimes and make it easier for stars to reionize the universe.

A surprising prediction from the BPASS models in Figure 23 is that the $\xi_{\text {ion }}$ values of 4-7 Myr populations do not decrease along the single-burst tracks of Figure 21. Rather, the WR stars in populations with binary evolution keep the $\xi_{\text {ion }}$ elevated, and nearly constant at the maximum $\xi_{\text {ion }}$ value, over the $10 \mathrm{Myr}$ lifetime of $\mathrm{O}$-stars. Comparing the inferred ionizing continua of RCS Knot E and S0108+0624 in Figure 16 demonstrates this behavior: the two galaxies have substantially different lightweighted ages but similar BPASS ionizing continua. Thus, the number of ionizing photons produced by a stellar population with binary evolution does not vary until the stellar population is older than $10 \mathrm{Myr}$. As mentioned above, this could substantially increase the total number of ionizing photons generated over the integrated lifetime of stellar populations within the epoch of reionization. This is also a testable observation of the effect of binary evolution using the nebular emission lines.

Determining the total number of ionizing photons intrinsically produced by a given stellar population is a key strength of FUV spectral synthesis. This analysis infers the total number of ionizing photons, as well as their spectral distribution, using observable spectral features from the same massive stars that emit the ionizing photons. In theory, spectral synthesis promises to be an extremely powerful tool for a wide assortment of extragalactic applications, from understanding the generation of nebular emission lines to uncovering the sources of cosmic reionization. In practice, determining the total number of ionizing photons requires precise constraints on the stellar population age, metallicity, star formation history, and the effect of binary star evolution (Figures 21 and 22). We demonstrated throughout this paper that massive star spectral features constrain the age, metallicity, and star formation history. However, we also emphasized that the non-ionizing FUV stellar continuum alone cannot constrain the effect of binary evolution. Binary evolution does not dramatically affect the number of ionizing photons produced per FUV luminosity of the youngest stellar populations $(<4 \mathrm{Myr})$, but it significantly increases the number of ionizing photons produced by older populations. Without constraining the effect of binary evolution, there is up to a factor of 7 uncertainty in the total number of ionizing photons produced by older stellar populations.

\section{Summary}

We have studied massive star populations in a sample of 61 starforming galaxies composed of 42 at $z<0.3$ from the HST archive and 19 at $z \sim 2$ from the MEGaSaURA sample. We fit a simple linear combination of single-age, fully theoretical stellar models to the far-ultraviolet stellar continuum $\left(\lambda_{\mathrm{r}}=1220-2000 \AA\right)$. From these fits, we derived light-weighted ages and metallicities of the stellar populations and extrapolated the fits to infer their ionizing continua $\left(\lambda_{\mathrm{r}}<912 \AA\right)$.

Individual stellar spectral features agree with the inferred light-weighted properties. The C IV stellar wind line varies with the inferred stellar age and metallicity in different and distinguishable ways (Figure 3), such that the C IV emission depends on stellar age (Figure 4) and the absorption depends on metallicity (Figure 5). The N V line strongly depends on age and is nearly independent of metallicity (Figure 6). Very young stellar populations have broad $\left(\sim 400 \mathrm{~km} \mathrm{~s}^{-1}\right)$ He II emission from WR stars, while older populations have negligible He II features (Figure 8). Finally, old populations have C III and Si III photospheric absorption features, while these features are undetected in younger populations (Figure 9).

The light-weighted stellar metallicity is strongly correlated with $(8 \sigma)$, and consistent with, the nebular metallicity (Figure 11). The metallicity relationship does not depend on redshift, as we find $z \sim 2$ stars and gas to have similar metallicities. Stellar metallicities may be the most reliable, yet time-consuming, method for inferring metallicities at high redshift (Section 5.1).

We used the stellar continuum models to demonstrate how the stellar ionizing continuum depends on stellar population properties (Section 5.2). Specifically, we characterized the ionizing continuum using three ratios of the ionizing flux density at different wavelengths to the non-ionizing flux density at $1500 \AA$. We focused on how the flux density ratio at $900-1500 \AA$, or $F_{900} / F_{1500}$, depends on stellar population properties, because $900 \AA$ is the most common wavelength used to observe the ionizing continuum. Theoretical stellar models demonstrate that the strength and spectral shape of the ionizing continuum of a single burst strongly depend on the stellar age (Figures 12 and 13) and metallicity (Figure 14). A multivariate relationship between $F_{900} / F_{1500}$ and both the age and metallicity (Equation (5)) describes most of the variation in $F_{900} / F_{1500}$ for a single burst of star formation (Figure 15 ).

We then inferred the ionizing continuum of the observed stellar populations by extrapolating the fits to the observed stellar continua. Stellar populations with older light-weighted 
ages have weaker inferred ionizing continua (Figure 16). However, only half (31 of 61) of the sample follows the singleburst $F_{900} / F_{1500}$ relations (Figure 17). The other half of the sample has a mixture of stellar populations at multiple ages. The mixed-age populations have stronger inferred ionizing continua at older ages than prescribed by a single-burst model. The inferred $F_{900} / F_{1500}$ of these galaxies is strongly linearly anticorrelated $(5.5 \sigma)$ with the light-weighted age (Equation (6)). These two populations are split at a light-weighted age of $8.6 \mathrm{Myr}$.

Mixed-age populations have stronger ionizing continua than single bursts of a similar age, due to the presence of very young stellar populations (Figure 17). We emphasized the differences between mixed-age populations and the typically assumed continuous star formation history (Section 5.3). Chiefly, the mixed-age continua depend on the relative mixture of age and metallicity. Thus, age-sensitive spectral features (C IV, $\mathrm{N} \mathrm{V}$, and He II) can vary, and the ionizing continuum can correspondingly increase or decrease. Mixed-age stellar populations produce significantly more ionizing photons than both a single burst with the same light-weighted age and a continuous star formation history, while having the same metallicity as the nebular gas (Figure 20). This is in stark contrast with previous works that assume a constant star formation history, and we emphasized that the only difference is the assumed star formation history.

Our fiducial stellar models are single-star STARBURST99 models mainly because these provided sufficient spectral resolution at all wavelengths (see the discussion in Section 3.2.2). We also fit the MEGaSaURA sample with stellar models that include binary evolution (BPASS models), but do not find significant differences between the STARBURST99 and BPASS fits to the FUV features (compare the blue and gold lines in Figures 3-6). Similarly, the light-weighted metallicities and ages are similar, as long as we only consider young ages for which the BPASS spectral resolution is adequate (Figure 18). The most significant difference is that BPASS models produce up to 12 times more high-energy ionizing photons than STARBURST99 models at older ages and lower metallicities (Figures 15 and 16). Consequently, the number of ionizing photons produced by a stellar population depends on the observationally unconstrained effect of binary evolution.

We provide scaling relations for the total number of ionizing photons produced per FUV luminosity $\left(\xi_{\text {ion }}\right)$. Note that $\xi_{\text {ion }}$ strongly depends on the age, metallicity, star formation history, and presence of binary stars (Figures 21 and 22). The inferred $\xi_{\text {ion }}$ values from our observations have an order of magnitude scatter that strongly depends on stellar population properties (Figure 23). We emphasize that $\xi_{\text {ion }}$ times the extinctioncorrected FUV luminosity determines the total number of ionizing photons produced by a stellar population. While fitting the FUV stellar continuum promises to be a powerful tool in determining the total number of ionizing photons produced by high-mass stars, without constraining the effect of binary evolution there is a factor of 7 uncertainty in the total number of ionizing photons produced by older stellar populations.
The rest-frame FUV spectral features of massive stars mirror the production of ionizing photons. Stellar population synthesis can estimate many properties required to infer the number of ionizing photons produced by stars and determine the source of cosmic reionization. This type of modeling is currently infeasible for galaxies within the epoch of reionization, but observations of the stellar continuum will be possible with future $30 \mathrm{~m}$ class telescopes and the James Webb Space Telescope. Stellar population synthesis of the most massive stars, as presented here, will be the most direct method to constrain the number of ionizing photons produced by the most distant star-forming galaxies.

We thank the anonymous referee for careful reading of the original manuscript and their insightful suggestions. We thank Chuck Steidel for kindly providing his stacked spectra for our comparison and detailed discussions. J.C. appreciates Christy Tremonti's early stellar continuum fitting guidance and discussions throughout this project. J.C. thanks Joe Cassinelli for early discussions that provided the fundamental stellar physics that inspired a substantial portion of this project. J.C. is grateful for helpful discussions and clarifications from Claus Leitherer. Discussions with Gwen Rudie also greatly improved the interpretation of this work. We thank X. Prochaska for insightful comments and clarifications that greatly improved the scope and clarity of the paper. J.C. thanks The Ohio State University for their hospitality while writing portions of this paper. J.R. is grateful for helpful discussions with George Sonneborn and Sally Heap. J.R. and D.B. acknowledge discussion and ideas presented at the Carnegie Symposium in honor of Leonard Searle, on the topic of "Understanding Nebular Emission in High-Redshift Galaxies," held at the Carnegie Observatories in Pasadena in 2015 July.

This paper includes data gathered with the $6.5 \mathrm{~m}$ Magellan Telescopes located at Las Campanas Observatory, Chile. We thank the staff of Las Campanas for their dedicated service, which has made possible these observations. We thank the telescope allocation committees of the Carnegie Observatories, The University of Chicago, The University of Michigan, Massachusetts Institute of Technology, and Harvard University for supporting the MEGaSaURA project over several years of observing. This paper includes data from observations made with the Nordic Optical Telescope, operated by the Nordic Optical Telescope Scientific Association at the Observatorio del Roque de los Muchachos, La Palma, Spain, of the Instituto de Astrofisica de Canarias.

\section{Appendix}

Here we give the tables of the derived stellar parameters for the MEGaSaURA (Table 3) and low-redshift samples (Table 4). We then give the tables of the ionization production efficiency $\left(\xi_{\text {ion }}\right)$ for both samples in Tables 5 and 6 . In the electronic version of the paper, we also include the models used to make the fits, the light fractions of each fit, and the fitted stellar continua for each galaxy. 


\begin{tabular}{|c|c|c|c|c|c|c|c|c|c|c|}
\hline $\begin{array}{l}\text { (1) } \\
\text { Galaxy Name }\end{array}$ & $\begin{array}{l}(2) \\
z\end{array}$ & $\begin{array}{c}(3) \\
E(B-V) \\
\text { SB99 } \\
(\mathrm{mag})\end{array}$ & $\begin{array}{c}(4) \\
E(B-V) \\
\text { BPASS } \\
(\mathrm{mag})\end{array}$ & $\begin{array}{c}(5) \\
Z_{\text {neb }} \\
\left(Z_{\odot}\right)\end{array}$ & $\begin{array}{c}(6) \\
Z_{*} \\
\text { SB99 } \\
\left(Z_{\odot}\right)\end{array}$ & $\begin{array}{c}(7) \\
Z_{*} \\
\text { BPASS } \\
\left(Z_{\odot}\right)\end{array}$ & $\begin{array}{c}\text { (8) } \\
\text { Age } \\
\text { SB99 } \\
\text { (Myr) }\end{array}$ & $\begin{array}{c}\text { (9) } \\
\text { Age } \\
\text { BPASS } \\
\text { (Myr) }\end{array}$ & $\begin{array}{c}(10) \\
F_{900} / F_{1500} \\
\text { SB99 }\end{array}$ & $\begin{array}{c}(11) \\
\text { Spectral } \\
\text { References }\end{array}$ \\
\hline RCS-0327-1326 Knot E & 1.7034 & 0.31 & 0.29 & $0.34(\mathrm{c})$ & $0.24 \pm 0.03$ & $0.18 \pm 0.02$ & $2.54 \pm 0.14$ & $2.59 \pm 0.14$ & $1.55 \pm 0.08$ & $3,13,10$ \\
\hline Sunburst Arc Region 5 & 2.3709 & 0.15 & 0.15 & $\ldots$ & $0.55 \pm 0.04$ & $0.66 \pm 0.05$ & $2.92 \pm 0.08$ & $3.59 \pm 0.09$ & $1.14 \pm 0.05$ & 4,11 \\
\hline S003341.5+024217 & 2.3887 & 0.33 & 0.32 & $\ldots$ & $0.84 \pm 0.04$ & $0.74 \pm 0.03$ & $5.00 \pm 0.51$ & $3.53 \pm 0.36$ & $0.76 \pm 0.08$ & 10 \\
\hline RCS-0327-1326 Knot U & 1.7039 & 0.27 & 0.28 & 0.34 (c) & $0.29 \pm 0.07$ & $0.09 \pm 0.02$ & $5.03 \pm 1.44$ & $5.16 \pm 1.47$ & $1.07 \pm 0.31$ & $3,10,13$ \\
\hline S095738.7+050929 & 1.8204 & 0.17 & 0.17 & $\ldots$ & $0.17 \pm 0.05$ & $0.20 \pm 0.06$ & $7.36 \pm 1.64$ & $4.38 \pm 0.98$ & $0.52 \pm 0.20$ & 1,10 \\
\hline S090003.3+223408 & 2.0326 & 0.10 & 0.09 & 0.31 (a) & $0.38 \pm 0.02$ & $0.30 \pm 0.02$ & $7.39 \pm 0.41$ & $7.19 \pm 0.39$ & $0.39 \pm 0.03$ & 5,10 \\
\hline SPT0356 & 2.362 & 0.14 & 0.14 & $\cdots$ & $0.22 \pm 0.07$ & $0.16 \pm 0.05$ & $8.55 \pm 3.88$ & $5.71 \pm 2.59$ & $0.47 \pm 0.33$ & $\cdots$ \\
\hline S010842.2+062444 & 1.9102 & 0.18 & 0.20 & $\cdots$ & $0.50 \pm 0.03$ & $0.35 \pm 0.02$ & $8.71 \pm 1.06$ & $6.20 \pm 0.75$ & $0.53 \pm 0.09$ & 9,10 \\
\hline SPT2325 & 1.5799 & 0.17 & 0.17 & $\cdots$ & $0.27 \pm 0.03$ & $0.15 \pm 0.02$ & $9.24 \pm 2.42$ & $6.13 \pm 1.61$ & $1.36 \pm 0.54$ & $\cdots$ \\
\hline Cosmic Horseshoe & 2.3812 & 0.11 & 0.09 & $0.57(b)$ & $0.52 \pm 0.07$ & $0.20 \pm 0.03$ & $9.33 \pm 4.20$ & $11.91 \pm 5.35$ & $1.13 \pm 0.76$ & 2,10 \\
\hline SPT0142 & 2.674 & 0.19 & 0.17 & $\ldots$ & $0.23 \pm 0.04$ & $0.14 \pm 0.03$ & $10.15 \pm 3.73$ & $12.36 \pm 4.54$ & $1.06 \pm 0.65$ & $\ldots$ \\
\hline RCS-0327-1326 Knot G & 1.7039 & 0.24 & 0.24 & $0.34(\mathrm{c})$ & $0.29 \pm 0.04$ & $0.24 \pm 0.04$ & $10.90 \pm 1.77$ & $4.66 \pm 0.76$ & $0.97 \pm 0.34$ & $3,10,13$ \\
\hline S000451.7-010321 & 1.6811 & 0.33 & 0.30 & $0.25(\mathrm{~d})$ & $0.25 \pm 0.02$ & $0.15 \pm 0.01$ & $13.41 \pm 0.80$ & $10.60 \pm 0.63$ & $1.08 \pm 0.22$ & 9,10 \\
\hline S142954.9+120239 & 2.8245 & 0.18 & 0.20 & $\cdots$ & $0.64 \pm 0.05$ & $0.60 \pm 0.04$ & $15.38 \pm 2.68$ & $9.55 \pm 1.66$ & $0.68 \pm 0.30$ & 8,10 \\
\hline SPT0310 & 1.9960 & 0.27 & 0.29 & $\cdots$ & $0.23 \pm 0.04$ & $0.30 \pm 0.05$ & $15.66 \pm 2.87$ & $8.97 \pm 1.65$ & $0.67 \pm 0.37$ & $\cdots$ \\
\hline PSZ0441 & 1.8008 & 0.30 & 0.30 & $\cdots$ & $0.10 \pm 0.05$ & $0.39 \pm 0.18$ & $19.27 \pm 4.47$ & $11.19 \pm 2.59$ & $0.41 \pm 0.57$ & $\cdots$ \\
\hline S152745.1+065219 & 2.7623 & 0.33 & 0.32 & $<0.65$ (f) & $0.49 \pm 0.04$ & $0.36 \pm 0.03$ & $21.30 \pm 3.32$ & $12.84 \pm 2.00$ & $0.22 \pm 0.12$ & 6,10 \\
\hline S122651.3+215220 & 2.9260 & 0.13 & 0.13 & $\cdots$ & $0.34 \pm 0.02$ & $0.14 \pm 0.01$ & $26.10 \pm 1.29$ & $19.98 \pm 0.99$ & $0.31 \pm 0.09$ & 7,10 \\
\hline Cosmic Eye & 3.0735 & 0.38 & 0.36 & $0.81(\mathrm{e})$ & $0.65 \pm 0.02$ & $0.50 \pm 0.02$ & $28.62 \pm 0.80$ & $15.34 \pm 0.43$ & $0.15 \pm 0.03$ & 10,12 \\
\hline
\end{tabular}

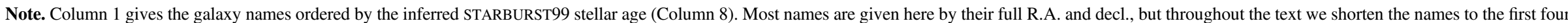

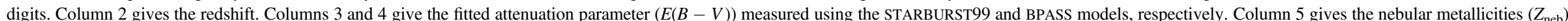

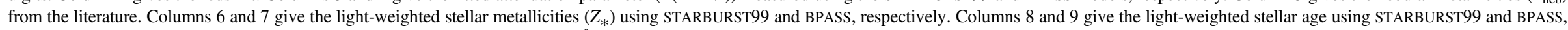

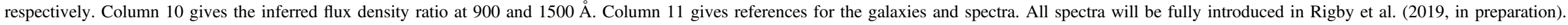
Metallicity references: (a) Bian et al. (2010), (b) Hainline et al. (2009), (c) Rigby et al. (2011), (d) Rigby et al. (2018a), (e) Stark et al. (2008), (f) Wuyts et al. (2012).

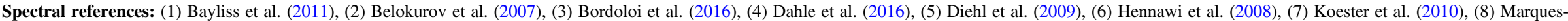
Chaves et al. (2017), (9) Rigby et al. (2014), (10) Rigby et al. (2018a), (11) Rivera-Thorsen et al. (2017, 2019), (12) Smail et al. (2007), (13) Wuyts et al. (2012). 
Table 4

Stellar Continuum Properties of the Low-redshift $H S T /$ COS Spectra

\begin{tabular}{|c|c|c|c|c|c|c|c|c|c|c|}
\hline $\begin{array}{l}\text { (1) } \\
\text { Galaxy Name }\end{array}$ & $\begin{array}{c}(2) \\
z\end{array}$ & $\begin{array}{c}(3) \\
E(B-V) \\
(\mathrm{mag})\end{array}$ & $\begin{array}{l}(4) \\
Z_{\text {neb }} \\
\left(Z_{\odot}\right)\end{array}$ & $\begin{array}{c}(5) \\
Z_{*} \\
\left(Z_{\odot}\right)\end{array}$ & $\begin{array}{c}(6) \\
\text { Age } \\
(\mathrm{Myr})\end{array}$ & $\begin{array}{c}(7) \\
F_{900} / F_{1500}\end{array}$ & $\begin{array}{l}(8) \\
\text { PID }\end{array}$ & $\begin{array}{c}(9) \\
Z_{\text {neb }} \\
\text { References }\end{array}$ & $\begin{array}{c}\quad(10) \\
\text { Metallicity } \\
\text { Calibration }\end{array}$ & $\begin{array}{c}(11) \\
\text { Spectra } \\
\text { References }\end{array}$ \\
\hline $\mathrm{J} 1416+1223$ & 0.1231 & 0.14 & 0.60 & $0.60 \pm 0.05$ & $1.88 \pm 0.35$ & $1.36 \pm 0.28$ & 13017 & Heckman et al. (2015) & PP04 & 1,6 \\
\hline $\mathrm{J} 0150+1260$ & 0.1467 & 0.12 & 0.50 & $0.52 \pm 0.13$ & $2.20 \pm 2.17$ & $1.23 \pm 1.25$ & 11727 & Heckman et al. (2015) & PP04 & 1,6 \\
\hline J0824+2806 & 0.0472 & 0.28 & 0.35 & $0.55 \pm 0.08$ & $2.48 \pm 3.12$ & $1.49 \pm 0.88$ & 13017 & Heckman et al. (2015) & PP04 & 1,6 \\
\hline J0907+5327 & 0.0299 & 0.08 & 0.22 & $0.56 \pm 0.19$ & $2.79 \pm 1.31$ & $1.23 \pm 0.72$ & 12583 & Östlin et al. (2014) & Direct & $5,9,10$ \\
\hline NGC 7552 & 0.0054 & 0.71 & 1.12 & $0.99 \pm 0.05$ & $2.88 \pm 0.04$ & $1.16 \pm 0.07$ & 12173 & Moustakas et al. (2011) & KK04 & 8 \\
\hline J0055-0021 & 0.1674 & 0.25 & 0.39 & $0.42 \pm 0.15$ & $3.16 \pm 2.27$ & $1.20 \pm 0.97$ & 11727 & Heckman et al. (2015) & PP04 & 1,6 \\
\hline $\mathrm{J} 1429+0643$ & 0.1736 & 0.20 & 0.27 & $0.36 \pm 0.09$ & $3.21 \pm 0.36$ & $1.11 \pm 0.30$ & 13017 & Heckman et al. (2015) & PP04 & 1,6 \\
\hline J0926+4427 & 0.1807 & 0.00 & 0.14 & $0.16 \pm 0.06$ & $3.24 \pm 0.37$ & $1.31 \pm 0.49$ & 11727 & Heckman et al. (2015) & PP04 & 1,6 \\
\hline J0808+3948 & 0.0912 & 0.15 & 1.12 & $1.46 \pm 0.04$ & $3.38 \pm 0.07$ & $0.91 \pm 0.02$ & 11727 & Heckman et al. (2015) & PP04 & 1,6 \\
\hline J0921+4509 & 0.2350 & 0.19 & 0.95 & $1.03 \pm 0.15$ & $3.73 \pm 0.62$ & $0.77 \pm 0.13$ & 11727 & Heckman et al. (2015) & PP04 & $1,2,6$ \\
\hline NGC 6090 & 0.0293 & 0.22 & 0.51 & $0.76 \pm 0.03$ & $3.77 \pm 0.11$ & $0.83 \pm 0.04$ & 12173 & Cortijo-Ferrero et al. (2017) & M13 & 8 \\
\hline $\mathrm{J} 0021+0052$ & 0.0984 & 0.10 & 0.32 & $0.45 \pm 0.13$ & $3.82 \pm 1.28$ & $1.06 \pm 0.30$ & 13017 & Heckman et al. (2015) & PP04 & 1,6 \\
\hline $\mathrm{J} 1415+0540$ & 0.0819 & 0.13 & 0.39 & $0.34 \pm 0.10$ & $3.91 \pm 2.40$ & $1.01 \pm 0.69$ & 13017 & Heckman et al. (2015) & PP04 & 1,6 \\
\hline NGC 3256 & 0.0094 & 0.49 & 1.10 & $0.57 \pm 0.12$ & $4.08 \pm 1.45$ & $0.76 \pm 0.31$ & 12173 & Engelbracht et al. (2008) & PP04 & 8 \\
\hline $\mathrm{J} 0213+1259$ & 0.2190 & 0.40 & 1.12 & $1.08 \pm 0.22$ & $4.09 \pm 1.65$ & $0.91 \pm 0.33$ & 13017 & Heckman et al. (2015) & PP04 & 1,6 \\
\hline KISSR 1578 & 0.0279 & 0.11 & 0.24 & $0.48 \pm 0.06$ & $4.11 \pm 2.03$ & $1.05 \pm 0.54$ & 11522 & Östlin et al. (2014) & Direct & 11 \\
\hline $\mathrm{J} 1112+5503$ & 0.1315 & 0.25 & 0.68 & $0.65 \pm 0.09$ & $4.38 \pm 3.54$ & $1.15 \pm 0.94$ & 13017 & Heckman et al. (2015) & PP04 & 1,6 \\
\hline NGC 4214 & 0.0010 & 0.30 & 0.32 & $0.33 \pm 0.06$ & $4.39 \pm 0.30$ & $0.95 \pm 0.20$ & 11579 & Kobulnicky \& Skillman (1996) & Direct & 7 \\
\hline $\mathrm{J} 1315+6207$ & 0.0308 & 0.43 & 1.02 & $0.97 \pm 0.17$ & $4.40 \pm 2.23$ & $0.75 \pm 0.40$ & 12583 & This work & Direct & $5,9,10$ \\
\hline NGC 7714 & 0.0093 & 0.42 & 0.60 & $0.84 \pm 0.02$ & $5.07 \pm 0.06$ & $0.51 \pm 0.01$ & 12604 & Gonzalez-Delgado et al. (1995) & Direct & 3 \\
\hline SBS $1415+437$ & 0.0020 & 0.14 & 0.08 & $0.08 \pm 0.02$ & $5.10 \pm 0.21$ & $0.81 \pm 0.19$ & 11579 & Thuan et al. (1999) & Direct & 7 \\
\hline KISSR 218 & 0.0209 & 0.46 & 1.51 & $1.20 \pm 0.19$ & $5.55 \pm 3.73$ & $1.03 \pm 0.71$ & 11522 & Salzer et al. (2005) & EP84 & 11 \\
\hline $\mathrm{J} 1429+1653$ & 0.1816 & 0.19 & 0.42 & $0.40 \pm 0.07$ & $6.58 \pm 2.73$ & $0.42 \pm 0.19$ & 13017 & Heckman et al. (2015) & PP04 & 1,6 \\
\hline $\mathrm{J} 0938+5428$ & 0.1021 & 0.11 & 0.21 & $0.24 \pm 0.08$ & $7.94 \pm 3.34$ & $1.07 \pm 0.58$ & 11727 & Heckman et al. (2015) & PP04 & 1,6 \\
\hline $\mathrm{J} 1025+3622$ & 0.1265 & 0.11 & 0.26 & $0.18 \pm 0.05$ & $8.69 \pm 3.64$ & $0.83 \pm 0.43$ & 13017 & Heckman et al. (2015) & PP04 & 1,6 \\
\hline KISSR 182 & 0.0224 & 0.32 & 0.45 & $0.53 \pm 0.17$ & $10.82 \pm 4.58$ & $0.71 \pm 0.38$ & 12027 & Salzer et al. (2005) & EP84 & 11 \\
\hline $\mathrm{J} 1525+0757$ & 0.0757 & 0.14 & 0.59 & $0.53 \pm 0.09$ & $11.04 \pm 5.18$ & $0.97 \pm 0.48$ & 13017 & Heckman et al. (2015) & PP04 & 1,6 \\
\hline Shoc 22 & 0.0179 & 0.10 & 0.23 & $0.28 \pm 0.05$ & $11.25 \pm 2.97$ & $0.74 \pm 0.23$ & 15099 & This work & Direct & $\cdots$ \\
\hline $\mathrm{J} 1250+0734$ & 0.0382 & 0.19 & 0.66 & $0.69 \pm 0.17$ & $13.83 \pm 6.80$ & $0.81 \pm 0.44$ & 12583 & Östlin et al. (2014) & Direct & $5,9,10$ \\
\hline MRK 1486 & 0.0338 & 0.12 & 0.13 & $0.10 \pm 0.05$ & $13.83 \pm 2.58$ & $0.79 \pm 0.46$ & 12583 & Östlin et al. (2014) & Direct & $5,9,10$ \\
\hline Haro 11 & 0.0206 & 0.09 & 0.40 & $0.38 \pm 0.02$ & $15.78 \pm 1.48$ & $0.77 \pm 0.08$ & 13017 & James et al. (2013) & Direct & 1,6 \\
\hline SBS $0926+606 \mathrm{~A}$ & 0.0137 & 0.14 & 0.21 & $0.32 \pm 0.08$ & $16.36 \pm 4.83$ & $0.72 \pm 0.28$ & 15099 & Berg et al. (2016) & Direct & $\cdots$ \\
\hline $\mathrm{J} 1307+5427$ & 0.0325 & 0.37 & 0.30 & $0.83 \pm 0.14$ & $16.75 \pm 4.87$ & $0.63 \pm 0.21$ & 12583 & This work & Direct & $5,9,10$ \\
\hline NGC 5253_1 & 0.0014 & 0.34 & 0.30 & $0.31 \pm 0.04$ & $17.13 \pm 2.43$ & $0.53 \pm 0.10$ & 11579 & Walsh \& Roy (1989) & Direct & 7 \\
\hline KISSR 108 & 0.0236 & 0.19 & 0.30 & $0.25 \pm 0.14$ & $17.96 \pm 5.12$ & $0.56 \pm 0.36$ & 12027 & Salzer et al. (2005) & EP84 & 11 \\
\hline $\mathrm{J} 1144+4012$ & 0.1270 & 0.26 & 0.51 & $0.41 \pm 0.10$ & $18.91 \pm 5.71$ & $0.51 \pm 0.20$ & 13017 & Heckman et al. (2015) & PP04 & 1,6 \\
\hline $1 \mathrm{Zw} 18$ & 0.0025 & 0.12 & 0.03 & $0.05 \pm 0.01$ & $20.78 \pm 0.71$ & $0.35 \pm 0.07$ & 11579 & Izotov et al. (1997) & Direct & 7 \\
\hline KISSR 242 & 0.0378 & 0.22 & 0.49 & $0.51 \pm 0.13$ & $21.73 \pm 5.16$ & $0.56 \pm 0.20$ & 11522 & Salzer et al. (2005) & EP84 & 4,11 \\
\hline IRAS 08339 & 0.0191 & 0.23 & 0.58 & $0.52 \pm 0.03$ & $22.98 \pm 1.22$ & $0.46 \pm 0.04$ & 12173 & López-Sánchez et al. (2006) & D02 & 8 \\
\hline NGC 4670 & 0.0036 & 0.26 & 0.32 & $0.37 \pm 0.04$ & $24.10 \pm 1.49$ & $0.41 \pm 0.05$ & 11579 & Heckman et al. (1998) & Direct & 7 \\
\hline NGC 4449 & 0.0007 & 0.37 & 0.42 & $0.41 \pm 0.03$ & $25.11 \pm 1.47$ & $0.33 \pm 0.03$ & 11579 & Marble et al. (2010) & Direct & 7 \\
\hline NGC 3690 & 0.0102 & 0.21 & 0.74 & $0.81 \pm 0.10$ & $28.16 \pm 1.07$ & $0.12 \pm 0.01$ & 11579 & Heckman et al. (1998) & Direct & 7 \\
\hline
\end{tabular}

Note. Fitted properties for the 42 low-redshift galaxies with COS/HST observations. Column 1 gives the galaxy name. Column 2 gives the galaxy redshift ( $z$ ). Column 3 gives the fitted stellar attenuation from the STARBURST99 fits to the stellar continua $(E(B-V))$. Column 4 gives the literature nebular metallicity of each galaxy $\left(Z_{\text {neb }}\right)$. Column 5 gives the inferred stellar metallicity from the STARBURST99 fits to the stellar continua $\left(Z_{*}\right)$. Column 6 gives the inferred stellar age from the STARBURST99 fits to the stellar continua. Column 7 is the inferred ratio of the flux at $900 \AA$ to the flux at $1500 \AA$. Column 8 is the $H S T$ project ID for each spectra. Column 9 is the literature reference for each $Z_{\text {neb }}$. Column 10 is the calibration method used to determine $Z_{\text {neb. }}$. Codes for metallicity calibration are as follows: PP04 (Pettini \& Pagel 2004), KK04 (Kobulnicky \& Kewley 2004), EP84 (Edmunds \& Pagel 1984), D02 (Denicoló et al. 2002), and M13 (Marino et al. 2013 ). Column 11 gives literature references for each spectra.

Spectral references: (1) Alexandroff et al. (2015), (2) Borthakur et al. (2014), (3) Fox et al. (2013), (4) France et al. (2010), (5) Hayes et al. (2014), (6) Heckman et al. (2015), (7) James et al. (2014a), (8) Leitherer et al. (2014), (9) Östlin et al. (2014), (10) Rivera-Thorsen et al. (2015), (11) Wofford et al. (2013). 
Table 5

$\xi_{\text {ion }}$ Values for the MEGaSaURA Sample

\begin{tabular}{lcc}
\hline \hline (1) & $(2)$ & $(3)$ \\
Galaxy Name & $\begin{array}{c}\log \left(\xi_{\text {ion }}\right) \text { SB } 99 \\
\log \left(\left[\text { photon } \AA \text { erg }^{-1}\right]\right)\end{array}$ & $\begin{array}{c}\log \left(\xi_{\text {ion }}\right) \text { BPASS } \\
\log \left(\left[\text { photon } \AA \mathrm{erg}^{-1}\right]\right)\end{array}$ \\
\hline RCS-0327-1326 Knot E & $13.53 \pm 0.03$ & $13.52 \pm 0.03$ \\
Sunburst Arc Region 5 & $13.29 \pm 0.02$ & $13.47 \pm 0.02$ \\
S003341.5+024217 & $13.11 \pm 0.05$ & $13.48 \pm 0.05$ \\
RCS-0327-1326 Knot U & $13.40 \pm 0.16$ & $13.50 \pm 0.16$ \\
S095738.7+050929 & $12.91 \pm 0.20$ & $13.53 \pm 0.21$ \\
S090003.3+223408 & $12.80 \pm 0.05$ & $13.36 \pm 0.05$ \\
SPT0356 & $12.95 \pm 0.37$ & $13.44 \pm 0.38$ \\
S010842.2+062444 & $12.93 \pm 0.09$ & $13.49 \pm 0.10$ \\
SPT2325 & $13.56 \pm 0.21$ & $13.52 \pm 0.21$ \\
Cosmic Horseshoe & $13.48 \pm 0.36$ & $13.30 \pm 0.35$ \\
SPT0142 & $13.38 \pm 0.33$ & $13.30 \pm 0.33$ \\
RCS-0327-1326 Knot G & $13.38 \pm 0.19$ & $13.50 \pm 0.19$ \\
S000451.7-010321 & $13.48 \pm 0.11$ & $13.42 \pm 0.11$ \\
S142954.9+120239 & $13.22 \pm 0.23$ & $13.35 \pm 0.24$ \\
SPT0310 & $13.07 \pm 0.29$ & $13.49 \pm 0.30$ \\
PSZ0441 & $12.86 \pm 0.75$ & $13.43 \pm 0.79$ \\
S152745.1+065219 & $12.42 \pm 0.29$ & $13.30 \pm 0.31$ \\
S122651.3+215220 & $12.74 \pm 0.16$ & $13.04 \pm 0.16$ \\
Cosmic Eye & $12.23 \pm 0.10$ & $13.10 \pm 0.11$ \\
\hline
\end{tabular}

Note. Photon production efficiency $\left(\xi_{\text {ion }}\right)$ for the MEGaSaURA sample. Column 2 gives the values inferred using the STARBURST99 models, and Column 3 gives the values for the BPASS models. The galaxies are listed in descending light-weighted age inferred using the STARBURST99 models.

Table 6

$\xi_{\text {ion }}$ Values for the Low-redshift $H S T /$ COS Sample

\begin{tabular}{|c|c|}
\hline $\begin{array}{l}\text { (1) } \\
\text { Galaxy Name }\end{array}$ & $\begin{array}{c}(2) \\
\log \left(\xi_{\text {ion }}\right) \\
\log \left(\left[\text { photon } \AA \operatorname{erg}^{-1}\right]\right)\end{array}$ \\
\hline $\mathrm{J} 1416+1223$ & $13.54 \pm 0.11$ \\
\hline $\mathrm{J} 0150+1260$ & $13.48 \pm 0.54$ \\
\hline J0824+2806 & $13.57 \pm 0.32$ \\
\hline $\mathrm{J} 0907+5327$ & $13.43 \pm 0.31$ \\
\hline NGC 7552 & $13.18 \pm 0.03$ \\
\hline J0055-0021 & $13.35 \pm 0.43$ \\
\hline $\mathrm{J} 1429+0643$ & $13.33 \pm 0.14$ \\
\hline J0926+4427 & $13.47 \pm 0.20$ \\
\hline $\mathrm{J} 0808+3948$ & $13.25 \pm 0.01$ \\
\hline J0921+4509 & $13.09 \pm 0.09$ \\
\hline NGC 6090 & $13.14 \pm 0.03$ \\
\hline $\mathrm{J} 0021+0052$ & $13.34 \pm 0.15$ \\
\hline $\mathrm{J} 1415+0540$ & $13.27 \pm 0.36$ \\
\hline NGC 3256 & $13.08 \pm 0.22$ \\
\hline $\mathrm{J} 0213+1259$ & $13.34 \pm 0.19$ \\
\hline KISSR 1578 & $13.34 \pm 0.27$ \\
\hline $\mathrm{J} 1112+5503$ & $13.47 \pm 0.44$ \\
\hline NGC 4214 & $13.18 \pm 0.11$ \\
\hline $\mathrm{J} 1315+6207$ & $13.10 \pm 0.29$ \\
\hline NGC 7714 & $12.89 \pm 0.01$ \\
\hline SBS $1415+437$ & $13.14 \pm 0.13$ \\
\hline KISSR 218 & $13.26 \pm 0.37$ \\
\hline $\mathrm{J} 1429+1653$ & $12.77 \pm 0.24$ \\
\hline $\mathrm{J} 0938+5428$ & $13.43 \pm 0.29$ \\
\hline $\mathrm{J} 1025+3622$ & $13.19 \pm 0.27$ \\
\hline KISSR 182 & $13.07 \pm 0.28$ \\
\hline $\mathrm{J} 1525+0757$ & $13.35 \pm 0.27$ \\
\hline Shoc 22 & $13.22 \pm 0.17$ \\
\hline $\mathrm{J} 1250+0734$ & $13.23 \pm 0.29$ \\
\hline MRK 1486 & $13.17 \pm 0.31$ \\
\hline Haro 11 & $13.29 \pm 0.06$ \\
\hline
\end{tabular}

Table 6

(Continued)

\begin{tabular}{lc}
\hline \hline (1) & $\begin{array}{c}(2) \\
\log \left(\xi_{\text {ion }}\right)\end{array}$ \\
& $\log \left(\left[\operatorname{photon} \AA \operatorname{erg}^{-1}\right]\right)$ \\
\hline SBS 0926+606A & $13.11 \pm 0.21$ \\
J1307+5427 & $13.05 \pm 0.18$ \\
NGC 5253_1 & $12.92 \pm 0.10$ \\
KISSR 108 & $13.02 \pm 0.34$ \\
J1144+4012 & $12.96 \pm 0.20$ \\
1 Zw 18 & $12.76 \pm 0.11$ \\
KISSR 242 & $13.05 \pm 0.19$ \\
IRAS 08339 & $13.00 \pm 0.05$ \\
NGC 4670 & $12.78 \pm 0.07$ \\
NGC 4449 & $12.69 \pm 0.05$ \\
NGC 3690 & $12.46 \pm 0.07$ \\
& \\
\hline
\end{tabular}

Note. Values of the ionizing photon production efficiency $\left(\xi_{\text {ion }}\right)$ for the lowredshift $H S T / C O S$ galaxies calculated using the STARBURST99 models. The table is in descending age order.

\section{ORCID iDs}

J. Chisholm (ib https://orcid.org/0000-0002-0302-2577

J. R. Rigby (i) https://orcid.org/0000-0002-7627-6551

M. Bayliss (iD https://orcid.org/0000-0003-1074-4807

D. A. Berg (1D https://orcid.org/0000-0002-4153-053X

H. Dahle (iD https://orcid.org/0000-0003-2200-5606

K. Sharon (iD https://orcid.org/0000-0002-7559-0864

\section{References}

Abbott, D. C., \& Conti, P. S. 1987, ARA\&A, 25, 113

Alam, S., Albareti, F. D., Allende Prieto, C., et al. 2015, ApJS, 219, 12

Alexandroff, R. M., Heckman, T. M., Borthakur, S., Overzier, R., \& Leitherer, C. 2015, ApJ, 810, 104

Asplund, M., Grevesse, N., Sauval, A. J., \& Scott, P. 2009, ARA\&A, 47, 481

Baldwin, J. A., Ferland, G. J., Martin, P. G., et al. 1991, ApJ, 374, 580

Baldwin, J. A., Phillips, M. M., \& Terlevich, R. 1981, PASP, 93, 5

Bayliss, M. B., Gladders, M. D., Oguri, M., et al. 2011, ApJL, 727, L26

Belokurov, V., Evans, N. W., Moiseev, A., et al. 2007, ApJL, 671, L9

Berg, D. A., Erb, D. K., Auger, M. W., Pettini, M., \& Brammer, G. B. 2018, ApJ, 859, 164

Berg, D. A., Erb, D. K., Henry, R. B. C., Skillman, E. D., \& McQuinn, K. B. W. 2019, ApJ, 874, 93

Berg, D. A., Skillman, E. D., Henry, R. B. C., Erb, D. K., \& Carigi, L. 2016, ApJ, 827, 126

Bian, F., Fan, X., Bechtold, J., et al. 2010, ApJ, 725, 1877

Bordoloi, R., Rigby, J. R., Tumlinson, J., et al. 2016, MNRAS, 458, 1891

Borthakur, S., Heckman, T. M., Leitherer, C., \& Overzier, R. A. 2014, Sci, 346, 216

Bouret, J.-C., Lanz, T., Hillier, D. J., et al. 2003, ApJ, 595, 1182

Bouwens, R. J., Aravena, M., Decarli, R., et al. 2016, ApJ, 833, 72

Brinchmann, J., Charlot, S., Heckman, T. M., et al. 2004, arXiv:astro-ph/ 0406220

Bruzual, G., \& Charlot, S. 2003, MNRAS, 344, 1000

Byler, N., Dalcanton, J. J., Conroy, C., et al. 2018, ApJ, 863, 14

Calzetti, D., Armus, L., Bohlin, R. C., et al. 2000, ApJ, 533, 682

Cardelli, J. A., Clayton, G. C., \& Mathis, J. S. 1989, ApJ, 345, 245

Castor, J. I., Abbott, D. C., \& Klein, R. I. 1975, ApJ, 195, 157

Chabrier, G. 2003, PASP, 115, 763

Chisholm, J., Tremonti, C. A., Leitherer, C., Chen, Y., \& Wofford, A. 2016, MNRAS, 457, 3133

Choi, J., Conroy, C., \& Byler, N. 2017, ApJ, 838, 159

Conroy, C. 2013, ARA\&A, 51, 393

Conroy, C., Graves, G. J., \& van Dokkum, P. G. 2014, ApJ, 780, 33 
Conroy, C., \& van Dokkum, P. 2012, ApJ, 747, 69

Cortijo-Ferrero, C., Gonzalez-Delgado, R. M., Perez, E., et al. 2017, MNRAS, 467, 3898

Crowther, P. A. 2007, ARA\&A, 45, 177

Dahle, H., Aghanim, N., Guennou, L., et al. 2016, A\&A, 590, L4 de Mello, D. F., Leitherer, C., \& Heckman, T. M. 2000, ApJ, 530, 251

Denicoló, G., Terlevich, R., \& Terlevich, E. 2002, MNRAS, 330, 69

Diehl, H. T., Allam, S. S., Annis, J., et al. 2009, ApJ, 707, 686

Drew, J. E. 1989, ApJS, 71, 267

Edmunds, M. G., \& Pagel, B. E. J. 1984, MNRAS, 211, 507

Elbaz, D., Daddi, E., Le Borgne, D., et al. 2007, A\&A, 468, 33

Eldridge, J. J., \& Stanway, E. R. 2009, MNRAS, 400, 1019

Eldridge, J. J., Stanway, E. R., Xiao, L., et al. 2017, PASA, 34, e058

Engelbracht, C. W., Rieke, G. H., Gordon, K. D., et al. 2008, ApJ, 678, 804

López-Sánchez, Á. R., Esteban, C., \& García-Rojas, J. 2006, A\&A, 449, 997

Ferland, G. J., Porter, R. L., van Hoof, P. A. M., et al. 2013, RMxAA, 49, 137 Finkelstein, S. L., D'Aloisio, A., Paardekooper, J. P., et al. 2019, ApJ, 879, 36 Fox, A. J., Richter, P., Wakker, B. P., et al. 2013, ApJ, 772, 110

Fragos, T., Lehmer, B. D., Naoz, S., Zezas, A., \& Basu-Zych, A. 2013, ApJL, 776, L31

France, K., Nell, N., Green, J. C., \& Leitherer, C. 2010, ApJL, 722, L80

Georgy, C., Ekström, S., Meynet, G., et al. 2012, A\&A, 542, A29

Gladders, M. D., \& Yee, H. K. C. 2005, ApJS, 157, 1

Gonzalez-Delgado, R. M., Perez, E., Diaz, A. I., et al. 1995, ApJ, 439, 604

Götberg, Y., de Mink, S. E., \& Groh, J. H. 2017, A\&A, 608, A11

Götberg, Y., de Mink, S. E., Groh, J. H., et al. 2018, A\&A, 615, A78

Green, G. M., Schafly, E. F., Finkbeiner, D. P., et al. 2015, ApJ, 810, 25

Green, J. C., Froning, C. S., Osterman, S., et al. 2012, ApJ, 744, 60

Groh, J. H., Ekstrom, S., Georgy, C., et al. 2019, arXiv:1904.04009

Hainline, K. N., Shapley, A. E., Kornei, K. A., et al. 2009, ApJ, 701, 52

Hayes, M., Ostlin, G., Duval, F., et al. 2014, ApJ, 782, 6

Heckman, T. M., Alexandroff, R. M., Borthakur, S., Overzier, R., \& Leitherer, C. 2015, ApJ, 809, 147

Heckman, T. M., Robert, C., Leitherer, C., Garnett, D. R., \& van der Rydt, F. 1998, ApJ, 503, 646

Hennawi, J. F., Gladders, M. D., Oguri, M., et al. 2008, AJ, 135, 664

Hernandez, S., Larsen, S., Aloisi, A., et al. 2019, arXiv:1901.08063

Howarth, I. D., \& Prinja, R. K. 1989, ApJS, 69, 527

Huber, P. J. 1981, Robust Statistics (New York: Wiley)

Izotov, Y. I., Thuan, T. X., \& Lipovetsky, V. A. 1997, ApJS, 108, 1

Izotov, Y. I., Worseck, G., Schaerer, D., et al. 2018, MNRAS, 478, 4851

James, B. L., Aloisi, A., Heckman, T., Sohn, S. T., \& Wolfe, M. A. 2014a, ApJ, 795, 109

James, B. L., Pettini, M., Christensen, L., et al. 2014b, MNRAS, 440, 1794

James, B. L., Tsamis, Y. G., Walsh, J. R., Barlow, M. J., \& Westmoquette, M. S. 2013, MNRAS, 430, 2097

Kehrig, C., Vílchez, J. M., Pérez-Montero, E., et al. 2015, ApJL, 801, L28

Kennicutt, R. C., \& Evans, N. J. 2012, ARA\&A, 50, 531

Kennicutt, R. C., Jr. 1998, ARA\&A, 36, 189

Kewley, L. J., \& Ellison, S. L. 2008, ApJ, 681, 1183

Kinney, A. L., Bohlin, R. C., Calzetti, D., Panagia, N., \& Wyse, R. F. G. 1993, ApJS, 86, 5

Kobulnicky, H. A., \& Kewley, L. J. 2004, ApJ, 617, 240

Kobulnicky, H. A., \& Skillman, E. D. 1996, ApJ, 471, 211

Kobulnicky, H. A., \& Skillman, E. D. 1997, ApJ, 489, 636

Koester, B. P., Gladders, M. D., Hennawi, J. F., et al. 2010, ApJL, 723, L73

Kroupa, P. 2001, MNRAS, 322, 231

Kudritzki, R. P. 1998, in Stellar Astrophysics for the Local Group: VIII Canary Islands Winter School of Astrophysics, ed. A. Aparicio, A. Herrero, \& F. Sánchez (New York: Cambridge Univ. Press), 149

Kudritzki, R.-P., \& Puls, J. 2000, ARA\&A, 38, 613

Kudritzki, R.-P., Urbaneja, M. A., Gazak, Z., et al. 2012, ApJ, 747, 15

Lamers, H. J. G. L. M., \& Cassinelli, J. P. 1999, Introduction to Stellar Winds (Cambridge: Cambridge Univ. Press)

Lamers, H. J. G. L. M., \& Leitherer, C. 1993, ApJ, 412, 771

Lamers, H. J. G. L. M., Snow, T. P., \& Lindholm, D. M. 1995, ApJ, 455, 269

Le Borgne, J.-F., Bruzual, G., Pello, R., et al. 2003, A\&A, 402, 433

Leitherer, C., Byler, N., Lee, J. C., \& Levesque, E. M. 2018, ApJ, 865, 55

Leitherer, C., Chapman, J. M., \& Koribalski, B. 1995, ApJ, 450, 289

Leitherer, C., Ekström, S., Meynet, G., et al. 2014, ApJS, 212, 14

Leitherer, C., Ortiz Otálvaro, P. A., Bresolin, F., et al. 2010, ApJS, 189, 309

Leitherer, C., Robert, C., \& Drissen, L. 1992, ApJ, 401, 596

Leitherer, C., Schaerer, D., Goldader, J. D., et al. 1999, ApJS, 123, 3

Leitherer, C., Tremonti, C. A., Heckman, T. M., \& Calzetti, D. 2011, AJ, 141,37
Levesque, E. M., Leitherer, C., Ekstrom, S., Meynet, G., \& Schaerer, D. 2012, ApJ, 751, 67

Madau, P., \& Dickinson, M. 2014, ARA\&A, 52, 415

Madau, P., Haardt, F., \& Rees, M. J. 1999, ApJ, 514, 648

Madau, P., Pozzetti, L., \& Dickinson, M. 1998, ApJ, 498, 106

Maraston, C. 2005, MNRAS, 362, 799

Marble, A. R., Engelbracht, C. W., van Zee, L., et al. 2010, ApJ, 715, 506

Marino, R. A., Rosales-Ortega, F. F., Sanchez, S. F., et al. 2013, A\&A, 559, A114

Markwardt, C. B. 2009, in ASP Conf. Ser. 411, Astronomical Data Analysis Software and Systems XVIII, ed. D. A. Bohlender, D. Durand, \& P. Dowler (San Francisco, CA: ASP), 251, arXiv:0902.2850

Marques-Chaves, R., Perez-Fournon, I., Shu, Y., et al. 2017, ApJL, 834, L18

Marshall, J. L., Burles, S., Thompson, I. B., et al. 2008, Proc. SPIE, 7014, 701454

McQuinn, K. B. W., Skillman, E. D., Cannon, J. M., et al. 2010a, ApJ, 721,297

McQuinn, K. B. W., Skillman, E. D., Cannon, J. M., et al. 2010b, ApJ, 724, 49

Meynet, G., \& Maeder, A. 2000, A\&A, 361, 101

Meynet, G., Maeder, A., Schaller, G., Schaerer, D., \& Charbonnel, C. 1994, A\&AS, 103, 97

Mirabel, I. F., Dijkstra, M., Laurent, P., Loeb, A., \& Pritchard, J. R. 2011 A\&A, 528, A149

Moustakas, J., Zaritsky, D., Brown, M., et al. 2011, arXiv:1112.3300

Nanayakkara, T., Brinchmann, J. \& The MUSE Collaboration 2018, arXiv: 1809.10970

Nemry, F., Surdej, J., \& Hernaiz, A. 1991, A\&A, 247, 469

Nieva, M.-F., \& Przybilla, N. 2012, A\&A, 539, A143

Noeske, K. G., Weiner, B. J., Faber, S. M., et al. 2007, ApJL, 660, L43

Östlin, G., Hayes, M., Duval, F., et al. 2014, ApJ, 797, 11

Ouchi, M., Mobasher, B., Shimasaku, K., et al. 2009, ApJ, 706, 1136

Pauldrach, A. W. A., Hoffmann, T. L., \& Lennon, M. 2001, A\&A, 375, 161

Pauldrach, A. W. A., Kudritzki, R. P., Puls, J., \& Butler, K. 1990, A\&A, 228, 125

Pellerin, A., Fullerton, A. W., Robert, C., et al. 2002, ApJS, 143, 159

Pérez-Montero, E., \& Amorín, R. 2017, MNRAS, 467, 1287

Pettini, M., \& Pagel, B. E. J. 2004, MNRAS, 348, L59

Pettini, M., Rix, S. A., Steidel, C. C., et al. 2002, ApJ, 569, 742

Pettini, M., Steidel, C. C., Adelberger, K. L., Dickinson, M., \& Giavalisco, M. 2000, ApJ, 528, 96

Planck Collaboration, Ade, P. A. R., Aghanim, N., et al. 2014, A\&A, 571, A29

Planck Collaboration, Ade, P. A. R., Aghanim, N., et al. 2016, A\&A, 594, A27

Puls, J., Kudritzki, R.-P., Herrero, A., et al. 1996, A\&A, 305, 171

Reddy, N. A., Steidel, C. C., Pettini, M., \& Bogosavljević, M. 2016, ApJ, 828, 107

Rigby, J. R., Bayliss, M. B., Chisholm, J., et al. 2018b, ApJ, 853, 87

Rigby, J. R., Bayliss, M. B., Gladders, M. D., et al. 2014, ApJ, 790, 44

Rigby, J. R., Bayliss, M. B., Sharon, K., et al. 2018a, AJ, 155, 104

Rigby, J. R., \& Rieke, G. H. 2004, ApJ, 606, 237

Rigby, J. R., Wuyts, E., Gladders, M. D., Sharon, K., \& Becker, G. D. 2011, ApJ, 732, 59

Rivera-Thorsen, T. E., Dahle, H., Chisholm, J., et al. 2019, arXiv:1904.08186 Rivera-Thorsen, T. E., Hayes, M., Ostlin, G., et al. 2015, ApJ, 805, 14

Rivera-Thorsen, T. E., Östlin, G., Hayes, M., \& Puschnig, J. 2017, ApJ, 837, 29

Rix, S. A., Pettini, M., Leitherer, C., et al. 2004, ApJ, 615, 98

Robertson, B. E., Ellis, R. S., Furlanetto, S. R., \& Dunlop, J. S. 2015, ApJL, 802, L19

Robertson, B. E., Furlanetto, S. R., Schneider, E., et al. 2013, ApJ, 768, 71

Salpeter, E. E. 1955, ApJ, 121, 161

Salzer, J. J., Lee, J. C., Melbourne, J., et al. 2005, ApJ, 624, 661

Sana, H., de Mink, S. E., de Koter, A., et al. 2012, Sci, 337, 444

Sander, A., Shenar, T., Hainich, R., et al. 2015, A\&A, 577, A13

Sanders, R. L., Shapely, A. E., Kriek, M., et al. 2016, ApJL, 825, L23

Schaerer, D., Fragos, T., \& Izotov, Y. I. 2019, A\&A, 622, L10

Schaerer, D., Izotov, Y. I., Nakajima, K., et al. 2018, A\&A, 616, L14

Schaerer, D., \& Vacca, W. D. 1998, ApJ, 497, 618

Schaffer, K. K., Crawford, T. M., Aird, K. A., et al. 2011, ApJ, 743, 90

Seyfert, C. K. 1943, ApJ, 97, 28

Shapley, A. E., Steidel, C. C., Pettini, M., \& Adelberger, K. L. 2003, ApJ, 588,65

Sharon, K., Bayliss, M. B., Dahle, H., et al. 2019, arXiv:1904.05940

Smail, I., Swinbank, A. M., Richard, J., et al. 2007, ApJL, 654, L33

Smith, L. J., Norris, R. P. F., \& Crowther, P. A. 2002, MNRAS, 337, 1309

Stanway, E. R., \& Eldridge, J. J. 2018a, arXiv:1811.03856

Stanway, E. R., \& Eldridge, J. J. 2018b, MNRAS, 479, 75 
Stanway, E. R., Eldridge, J. J., \& Becker, G. D. 2016, MNRAS, 456, 485

Stanway, E. R., Eldridge, J. J., Greis, S. M. L., et al. 2014, MNRAS, 444, 3466

Stark, D. P., Swinbank, A. M., Ellis, R. S., et al. 2008, Natur, 455, 775

Steidel, C. C., Bogosavljević, M., Shapley, A. E., et al. 2018, ApJ, 869, 123

Steidel, C. C., Pettini, M., \& Adelberger, K. L. 2001, ApJ, 546, 665

Steidel, C. C., Strom, A. L., Pettini, M., et al. 2016, ApJ, 826, 159

Strom, A. L., Steidel, C. C., Rudie, G. C., et al. 2017, ApJ, 836, 164

Strom, A. L., Steidel, C. C., Rudie, G. C., Trainor, R. F., \& Pettini, M. 2018, ApJ, 868, 117

Strömgren, B. 1939, ApJ, 89, 526

Thuan, T. X., Izotov, Y. I., \& Foltz, C. B. 1999, ApJ, 525, 105

Tinsley, B. M. 1980, FCPh, 5, 287

Van Bever, J., \& Vanbeveren, D. 2000, A\&A, 358, 462

Vanbeveren, D., Van Bever, J., \& Belkus, H. 2007, ApJL, 662, L107
Villante, F. L., Serenelli, A. M., Delahaye, F., \& Pinsonneault, M. H. 2014, ApJ, 787, 13

Vink, J. S., de Koter, A., \& Lamers, H. J. G. L. M. 2001, A\&A, 369, 574

Wakker, B. P., Hernandez, A. K., French, D. M., et al. 2015, ApJ, 814, 40

Walborn, N. R., Fullerton, A. W., Crowther, P. A., et al. 2002, ApJS, 141, 443

Walborn, N. R., Nichols-Bohlin, J., \& Panek, R. J. 1985, NASRP, 1155

Walborn, N. R., \& Panek, R. J. 1984, ApJL, 280, L27

Walsh, J. R., \& Roy, J.-R. 1989, MNRAS, 239, 297

Westera, P., Lejeune, T., Buser, R., Cuisinier, F., \& Bruzual, G. 2002, A\&A, 381,524

Wofford, A., Leitherer, C., \& Salzer, J. 2013, ApJ, 765, 118

Wuyts, E., Rigby, J. R., Sharon, K., \& Gladders, M. D. 2012, ApJ, 755, 73

Yuan, T.-T., \& Kewley, L. J. 2009, ApJL, 699, L161 